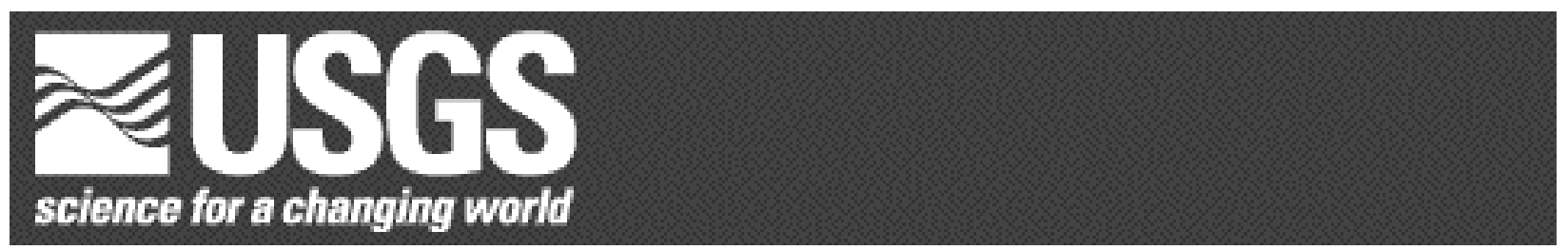

\title{
Locations and Descriptions of Gravity, Box, and Push Cores Collected in San Francisco Bay Between January and February, 1990 and 1991
}

\author{
By \\ Roberto J. Anima 1 , H. Edward Clifton ${ }^{2}$, Carol Reiss ${ }^{1}$ \\ and Florence L. Wong 1
}

Open File Report 2005-1453

Any use of trade, firm, or product names is for descriptive purposes only and does not imply endorsement by the U.S. Government.

U.S. DEPARTMENT OF THE INTERIOR

U.S. GEOLOGICAL SURVEY

${ }^{1}$ Menlo Park, CA 94025

${ }^{2}$ Menlo Park, CA 94025 (retired) 

ABSTRACT.

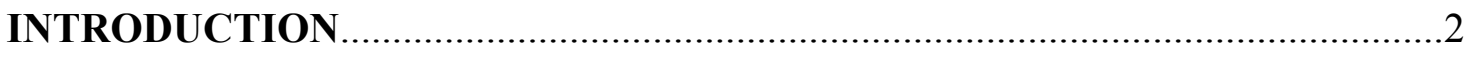

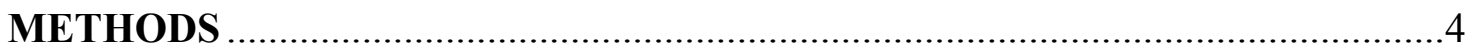

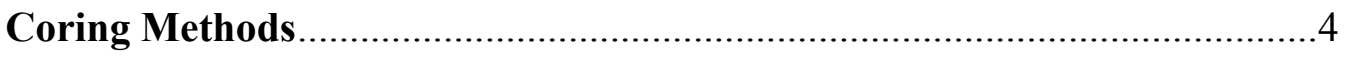

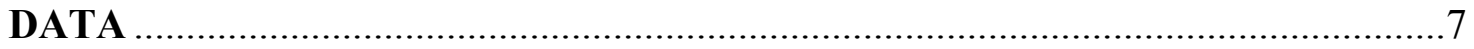

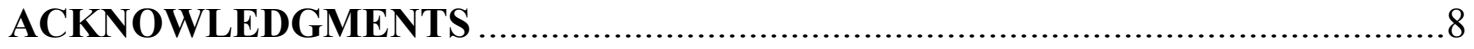

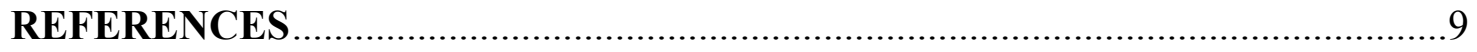

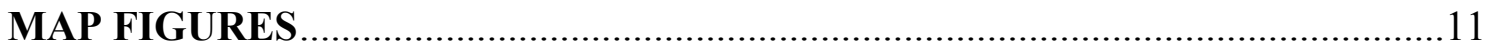

Figure 1 Map of San Francisco Bay and Delta area showing core sites

with inset detail maps A through Q. ..................................................12

Figure 2 Detailed map of Area A showing locations of cores collected in

South San Francisco Bay between the confluences of Guadalupe

River and Coyote Creek..................................................................13

Figure 3 Detailed map of Area B showing locations of cores collected

South of the Dumbarton Bridge near the mouth of Newark Slough........14

Figure 4 Detailed map of Area C showing locations of cores collected

north of the Dumbarton Bridge across the main channel......................15

Figure 5 Detailed map of Area D showing locations of cores collected

north and south of the mouth of Redwood Creek, across the main

channel and subtidal flats............................................................. 16

Figure 6 Detailed map of Area E showing locations of cores collected

south of the San Mateo Bridge across the main channel.

Figure 7 Detailed map of Area F showing locations of cores collected in

South San Francisco Bay across the main channel and subtidal

flats. 
Figure 8 Detailed map of Area $\mathrm{G}$ showing locations of cores collected in South-central San Francisco Bay between the San Francisco-

Oakland Bay Bridge and San Mateo Bridge.

Figure 12 Detailed map of Area J showing locations of cores collected in

San Rafael and San Pablo Bays, the Petaluma River to the

Carquinez Bridge.

Figure 13 Detailed map of Area K showing locations of cores collected in Carquinez Strait. Note detailed map adjacent to Southampton Bay.......23

Figure 14 Detailed map of Area L showing locations of cores collected between Dillon Point and the city wharf at Benicia.

Figure 15 Detailed map of Area M showing locations of cores collected in Suisun Bay east of the Martinez-Benicia Bridge and Chipps Island, and in Suisun Slough.

Figure 16 Detailed map of Area N showing locations of cores collected along the confluence of the Sacramento and San Joaquin Rivers.

Figure 17 Detailed map of Area $\mathrm{O}$ showing locations of cores collected along the Sacramento River from Sherman to Decker Islands. 27

Figure 18 Detailed map of Area P showing locations of cores collected along the San Joaquin River between Three Mile Slough and Seven mile Slough. .28

Figure 19 Detailed map of Area Q showing locations of cores collected on the Sacramento River northeast of Rio Vista to Steamboat and Cache Sloughs 29

CORE TABLES 30

1990 Cores 31

South San Francisco Bay. 31

South of the Dumbarton Bridge 31 
Between the San Mateo and Dumbarton Bridge

San Joaquin River

Sacramento River from Northeast of Rio Vista to the San Joaquin

River

Suisun Bay in the vicinity of Roe and Ryer Island

San Joaquin River near the confluence with the Sacramento River area of Fraser Shoal

Suisun Bay, South of Ryer Island to Seal Island

Carquinez Straits

San Pablo Bay from the mouth of the Napa River to Sisters Rocks and

Petaluma River.

Richmond San Rafael Bay from Pt. San Pedro to South of Paradise

Cay. .40

Richardson Bay 41

Central Bay Off Richmond and Berkeley Marinas

South San Francisco Bay

South and adjacent to the San Mateo Bridge.

North of the San Mateo Bridge to Potrero Point and Alameda outer harbor

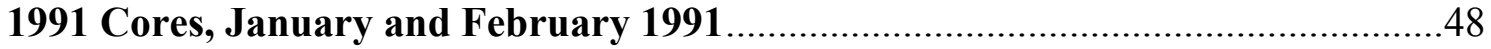

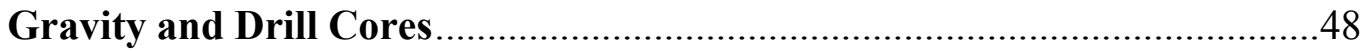

Sacramento to the mouth of the San Joaquin River .......................48

Suisun Bay to Benicia Bridge ......................................................49

San Joaquin River to the confluence with the Sacramento ..............50

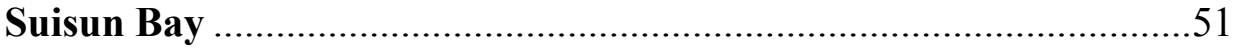

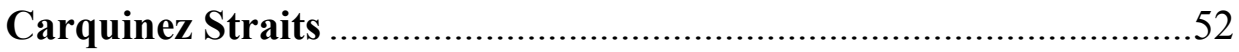

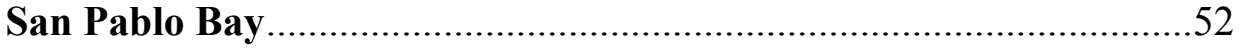




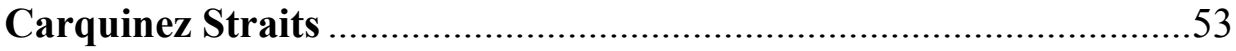

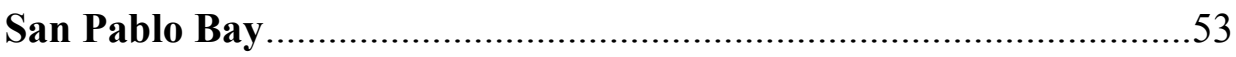

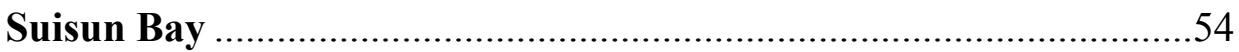

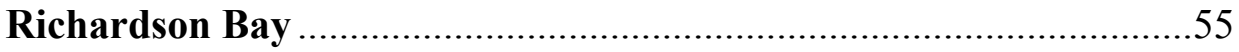

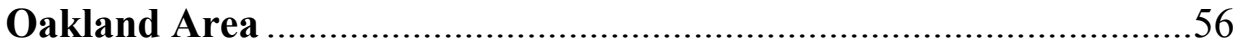

Push Cores Collected at the Mouth of Richardson Bay ..............................57

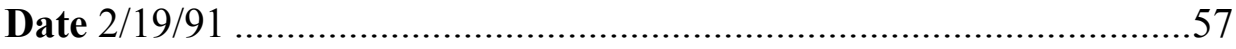

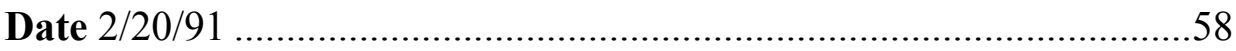

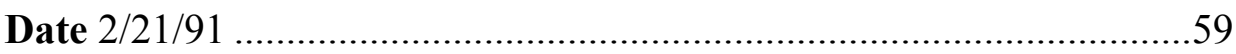

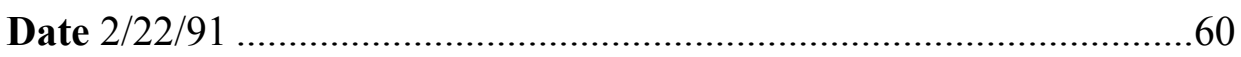

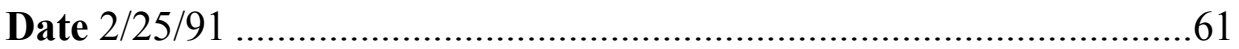

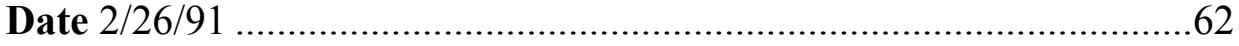

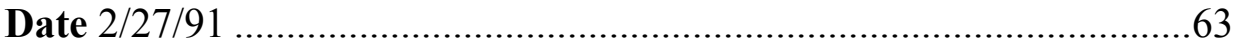


- v - 


\begin{abstract}
A project to study San Francisco Bay sediments collected over 300 sediment gravity cores; six push cores, and 3 box cores in San Francisco Bay during the years 1990-91. The purpose of the sampling effort is to establish a database on the Holocene sediment history of the bay. The samples described and mapped are the first effort to catalog and present the data collected. Thus far the cores have been utilized in various cooperative studies with state colleges and universities, and other USGS divisions. These cores serve as a base for ongoing multidisciplinary studies. The sediment studies project has initiated subsequent coring efforts within the bay using refined coring techniques to attain deeper cores.
\end{abstract}




\section{INTRODUCTION}

The initial intent of this project was to focus on attaining sediment gravity cores in as many varying depositional environments within the San Francisco Bay and Delta as possible to establish a data base on the Holocene sedimentary history of San Francisco Bay. This report describes cores collected over four cruises conducted in 1990 and 1991. The first cruise of 1990 focused on the area south of the Dumbarton Bridge in South San Francisco Bay and the Sacramento and San Joaquin rivers to Suisun Bay (http://walrus.wr.usgs.gov/infobank/j/j190sf/html/j-1-90-sf.meta.html). The second cruise in 1990 collected cores from Suisun Bay, San Pablo Bay, Central Bay, and South Bay (http://walrus.wr.usgs.gov/infobank/j/j290sf/html/j-2-90-sf.meta.html). These two cruises occupied a total of 200 core sites. Although not all the sites relinquished a core, sediment samples were attained from the core catcher at each of the sites.

The 1991 cruises focused on areas missed or overlooked during the first year's cruises. The first cruise of 1991 collected cores along the Sacramento River and the confluence of the Sacramento and San Joaquin Rivers, Suisun Bay, Carquinez Strait, and San Pablo Bay (http://walrus.wr.usgs.gov/infobank/j/j191sf/html/j-1-91-sf.meta.html). The second cruise of 1991 focused on the mouth of Richardson Bay, and east of Treasure Island (http://walrus.wr.usgs.gov/infobank/j/j291sf/html/j-2-91-sf.meta.html). A total of 79 cores, including 6 push cores, were collected in Richardson Bay in 1991.

This report describes the methods used to acquire cores and presents core location maps and accompanying core description tables that contain information gathered during the coring operations. The cores have been used for grain size analysis, engineering properties analysis, microfaunal investigation, and geochemical analysis. Analyses of the cores have raised questions that have resulted in further microfaunal, geochemical, and geophysical studies of the bay (Van Geen and others,1991; Luoma and others, 1992 \& 
1996; Anima, 1994a, b, \& 1995; Hornberger and others, 1997, Pereira and others, 1997; Hostettler and others, 1997; Fuller and others, 1997).

The tables of core number, location, length, water depth, and notes are based on the onboard data collection notes taken during the coring process. Unless otherwise noted, the gravity coring was conducted using a $600 \mathrm{lb}$ weight stand. In some instances the table lacks information in one or more categories; this is due to data not being noted at the time of collection. At some locations the coring device was not able to penetrate the bottom or able to retain the core due to either the bottom type or a malfunction of the equipment. In these instances, the core cutter-catcher often did manage to collect a small portion of the surface material; this small piece of material was bagged and saved.

Core locations were obtained through either radar fixes both on land and navigation markers or through Mini-Ranger or Del Norte microwave antenna positions located onshore with distances being recorded onboard the vessel. Depth measurements were obtained using a hull mounted $200 \mathrm{kHz}$ Raytheon DE719 Fathometer. 


\section{METHODS}

\section{Coring Methods}

Gravity coring, box coring and push coring were conducted in San Francisco Bay and Delta during January and February of 1990 and 1991. The work utilized the vessel R/V David Johnston on loan to the University of California Santa Cruz. The David Johnston is 43 feet in length equipped with a hydraulically operated A-frame and winch capable of lifting 2 tons. Standard gravity coring techniques utilized a $600 \mathrm{lb}$.

(kilograms) weight stand and 4, 8, 10, and $12 \mathrm{ft}(1.2 \mathrm{~m}, 2.4 \mathrm{~m}, 3.0 \mathrm{~m}$ and $3.6 \mathrm{~m})$ core barrels. Box coring used a Soutar Van Veen box corer that is scaled down to 3/4 the size of a normal Soutar corer. This corer was designed for the University of Southern California and purchased by the U.S. Geological Survey to collect undisturbed cores related to infauna studies of the bay sediments. The push coring was a cooperative effort between the U.S. Geological Survey and the University of Mississippi Minerals Technology Center who supplied the coring equipment allowing the collection of continuous cores to a depth of up to 21 meters.

The $600 \mathrm{lb}$. gravity core weight stand was attached to a core barrel using a quick release pin. Clear 3 in $(7.6 \mathrm{~cm})$ polybuterate core liner was cut to length to fit inside the

core barrel with a core catcher and cutting head attached by screws to the leading edge of the core barrel. The core barrel, with core liner, catcher , and cutter, was attached to the weight stand and lowered to approximately 6 to $10 \mathrm{ft}$ (2-3 meters) above the bay floor where possible. From that height the core was allowed to free-fall. Experience showed that to free-fall the corer from a higher distance would cause the corer to hit the bottom at an angle. This could, and did on one occasion, result in a bent and unusable core barrel. Once the corer was on the bottom and penetration made, the core barrel was pulled up and disconnected form the weight stand. Then, the core catcher and cutter were removed and the polybuterate core liner was then removed from the core barrel. The core liner 
was then capped using plastic vinyl caps with acetone as a sealant. The core liners were propped in a vertical position where residual seawater was drained from the top of the core. Excess core liner material from the top of the core was then cut off and the core measured for length, with visible physical characteristics, and other observations noted. At sites where little or no core penetration was made, samples of the material left in the core catcher was collected, bagged and marked.

Box coring was accomplished using a 3/4 size Soutar Van Veen corer, with box dimensions of 10 in x 14 in $x 20$ in $(25 \mathrm{~cm} \times 35 \mathrm{~cm} \times 50 \mathrm{~cm})$. The core box is a split casing held together with screws that allow for the sub sampling of the core into slabs that can then be X-radiographed.

The deep penetration coring, conducted only in Richardson Bay, used a 30' x 60' $(9 \mathrm{~m} \times 18 \mathrm{~m}$ ) barge on a three point anchoring system, with a 3 foot by 8 -foot ( $1 \mathrm{~m}$ by 2.4 m) moon pool in the middle of the barge. The coring operation used standard 3 in $(8 \mathrm{~cm})$ drill casing to case the upper 10 feet $(3.0 \mathrm{~m})$ of the core hole. The cores collected were 4 foot (1 meter) Shelby tubes with a 2 in $(5 \mathrm{~cm})$ diameter. The casing was lowered to the bottom and pushed into the sediment to a depth of 10 feet $(3.0 \mathrm{~m})$ with $10-15$ feet $(3-4.5$ m) of additional drill casing extending to the surface at the moon pool. The Shelby tube was then attached to a 1 in $(2.5 \mathrm{~cm})$ pipe threaded to the end of the Shelby tube and sections of the small diameter pipe added on to allow the Shelby tube to be pushed into the bottom. Once the Shelby tube was pushed into the sediment the tube was lifted and disconnected. A water pump connected to the 1 in $(2.5 \mathrm{~cm})$ pipe was once again lowered to the bottom of the hole to remove any sediment tailings left behind. Once the tailings were expelled from the top of the drill casing, the Shelby tube was once again lowered to recover the next 4-foot ( 1 meter) section. The depth of penetration was determined by measuring the number of lengths of 1-inch $(2.5 \mathrm{~cm})$ pipe added to the string. This operation was repeated for each 1-meter length of Shelby tube. The drill casing was cleaned with seawater pumped through the 1-inch $(2.5 \mathrm{~cm})$ pipe after each core was 
collected. Uncertainties arose when material was continuously being pumped out of the drill casing with no clearing of the material. The structure of the upper 2 in $(5 \mathrm{~cm})$ of sediment in the Shelby tubes when looked at with X-radiographs suggested that in some instances some of the overlying material did slough into the hole. This is supported by the discrepancies between $\mathrm{C}^{14}$ dating and the faunal assemblages found in the stratigraphy. Overall, the method worked well and did allow us to extract the stratigraphic information we were seeking, but, the system needs to be refined and more control needs to be developed for cleaner cores. 


\section{DATA}

This report outlines the locations, water depths, and amount of sub bottom penetration attained. Most of the cores have undergone X-radiography, some have been sub-sampling for grain size analysis, microfaunal identification, sediment geochemical analysis, radiometric dating, and stratigraphic interpretation. The maps show core numbers next to either a exclamation point (!), for 1990 cores, or a pound sign (\#), for 1991 cores and (s) for the push cores. The numbers correspond to numbers listed on the table for ease of presentation. The map letter (A through Q) that the cores are plotted on is located below the section title and location. The actual core numbers following the map number (Core Tables) are designated as San Francisco Bay (SFB) cores, with the month (01), the day (23), the year (91), and sequential number of the core collected for that particular day, i.e., SFB022190-3 for 1990 cores 39 to 200 . Cores 1 to 39, 1990, were labeled with a different sequence $1-\mathrm{G}-23,6-\mathrm{G}-26$, etc. The setting of the core is based on general area of the sample location and distances to known range marks or land forms in nautical miles (nm), water depth is in meters (m) or feet (ft. or '), core type is either gravity (G), deep penetration (D), Grab (grab), or box core (B). The core length is in meters and the comments describe observations from the actual coring operation and any distinctive information that would aid in working up the final data about each core. The push core locations, at the end of the Core Table, were plotted on the map (I).

The core information is being digitized and put into tables, maps, and core descriptions. It also is being entered into a Geographic Information Systems (GIS). 


\section{ACKNOWLEDGMENTS}

The authors wish to thank those individuals who aided in obtaining the numerous samples or in the processing of the cores and maps. Thanks to Gordon Smith, boat driver, who got us into places we shouldn't have been. Also Greg Gable, Bill Webber, John Chin, Tom Reiss, Nathaniel Plank, Bruce Jaffe, Tom Clifton, Tom Lorenson were involved with the core collection at various times through the project. Armando Burciaga, a Minority Participation in Earth Sciences (MPES) student from UCLA who worked diligently on the cores, conducted a portion of the X-radiography and core description. Dave Britton, a summer volunteer helped with the core descriptions. The preliminary map illustration was done by Kristin Brown. The Marine Technician crew of Hal Williams, Ranson Rideout, and Kevin O'Toole aided in the preparation of the coring equipment. And thanks to the University of Mississippi Mineral Research Institute crew headed by Bob Woolsey, and Walter O'Niell, including Bob Sheldon and Brien Noakes for the use of their equipment and expertise in the push-coring part of the operation. 


\section{REFERENCES}

Anima, R.J., 1994a, Records of Contaminant input to San Francisco Bay: Core collection and sedimentology, Oral Presentation at the February 21-25, 1994 Ocean sciences Meeting of the American Geophysical Union, San Diego, Ca., in EOS, Transactions, American Geophysical Union, Vol. 75, No. 3.

Anima, R.J., 1994b, Carbon 14 dating of selected cores from San Francisco Bay, Ca., oral presentation given at the June 7, 1994 CONCERT, "Geologic Structures Beneath South San Francisco Bay - A Seismic Hazard?" Workshop, Austin Hall Auditorium, Bldg. 461, Treasure Island, Ca

Anima, R.J., Marlow, M.S., and Carlson, P., 1995, Variations in depositional settings based on shell layers found in gravity cores from south San Francisco Bay, CA: accepted to Pacific Section AAPG meeting, May 1995, AAPG Bull., v. 79, p. 578.

Fuller,C.C, Van Geen, A., Baskaran, M., Anima, R.J., 1997, Sediment chronology in San Francisco Bay California, defined by ${ }^{210} \mathrm{~Pb},{ }^{234} \mathrm{Th},{ }^{137} \mathrm{Cs}$, and $239,240 \mathrm{Pu}$. Marine Chemistry, Vol. 64 - NOS. 1-2, ISSN0304-4203 pgs. 7-27.

Horberger, M.I., Luoma, S.N., Van Geen, A., Fuller,C., Anima, R.J., 1997, Historical trends of trace metals in the sediments of San Francisco Bay, California. Marine Chemistry, Vol. 64 - NOS. 1-2, ISSN0304-4203 pgs. 39-55

Hostettler, F.D., Pereira, W.E., Kvenvolden, D.A., Van Geen, A., Luoma, S.N., Fuller,C.C, Anima, R.J., 1997, A Record of contaminant input to San Francisco Bay as traced by biomarker profiles in surface sediment and sediment cores. Marine Chemistry, Vol. 64 - NOS. 1-2, ISSN0304-4203 pgs. 115-127.

Luoma, S.N., Van Geen, A., Fuller, C., Hornberger, M., Pereira, W., Hostettler, F., Kvenvolden, K., Anima, R.J., Ritson, P., Flegal, A.R.,1996, Historical Contamination Trends in Sediments of San Francisco Bay.: American geophysical Union 1996 Fall Meeting, San Francisco, CA. Programs and Abstracts Vol. 77, No. 46, pg. 239

Luoma, S.N., Van Geen, A., Fuller, C.C, Hornberger, M., Anima, R.J., 1992, A record of $\mathrm{Cd}$ contamination in the water column of San Francisco Bay from $\mathrm{Cd} / \mathrm{Ca}$ ratios in foraminifera: Abstract, American Geophysical Union 1992 Fall Meeting San Francisco, CA. Programs and Abstracts Vol. 73, No. 43, pg. 304.

Pereira, W.E., Hostettler, F.D., Luoma, S.N., Van Geen, A., Fuller,C.C, Anima, R.J., 1997, Sedimentary record of anthropogenic and biogenic polycyclic aromatic hydrocarbons in San Francisco Bay, California. Marine Chemistry, Vol. 64 - NOS. 1-2, ISSN0304-4203 pgs. 99-113. 
Van Geen, A.C. Fuller, C. Brown, S. Luoma, Anima, R.J., E. Clifton, S. Trombore, D. Sloan, 1991, Cd/Ca Ratios in Foraminiferal Shells from San Francisco Bay: A Record of Estuarine Chemistry: 2nd International Symposium on Biogeochemistry of Model Estuaries "Estuarine Processes in Global Change" April 14-20, 1991, Jekyll Island, Georgia 


\section{MAP FIGURES}

USGS OF 2005-1453 


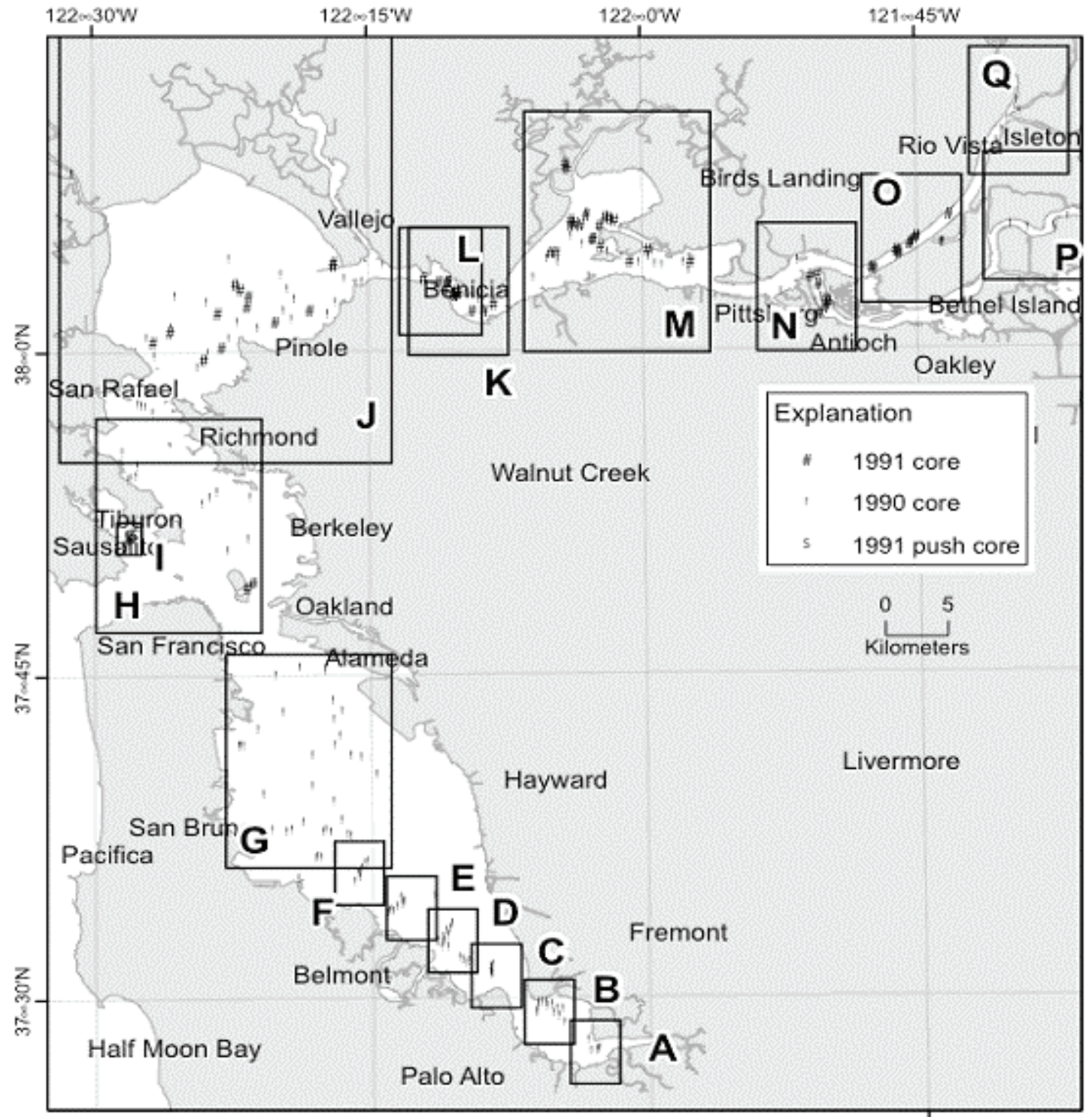

Figure 1 Map of San Francisco Bay and Delta area showing core sites with inset detail maps A through Q. Note that 1990 cores represented by exclamation points (!) and 1991 cores represented by pound signs (\#). The 1991 push cores are represented by an "s", and are only located in Richardson Bay (Map I). 


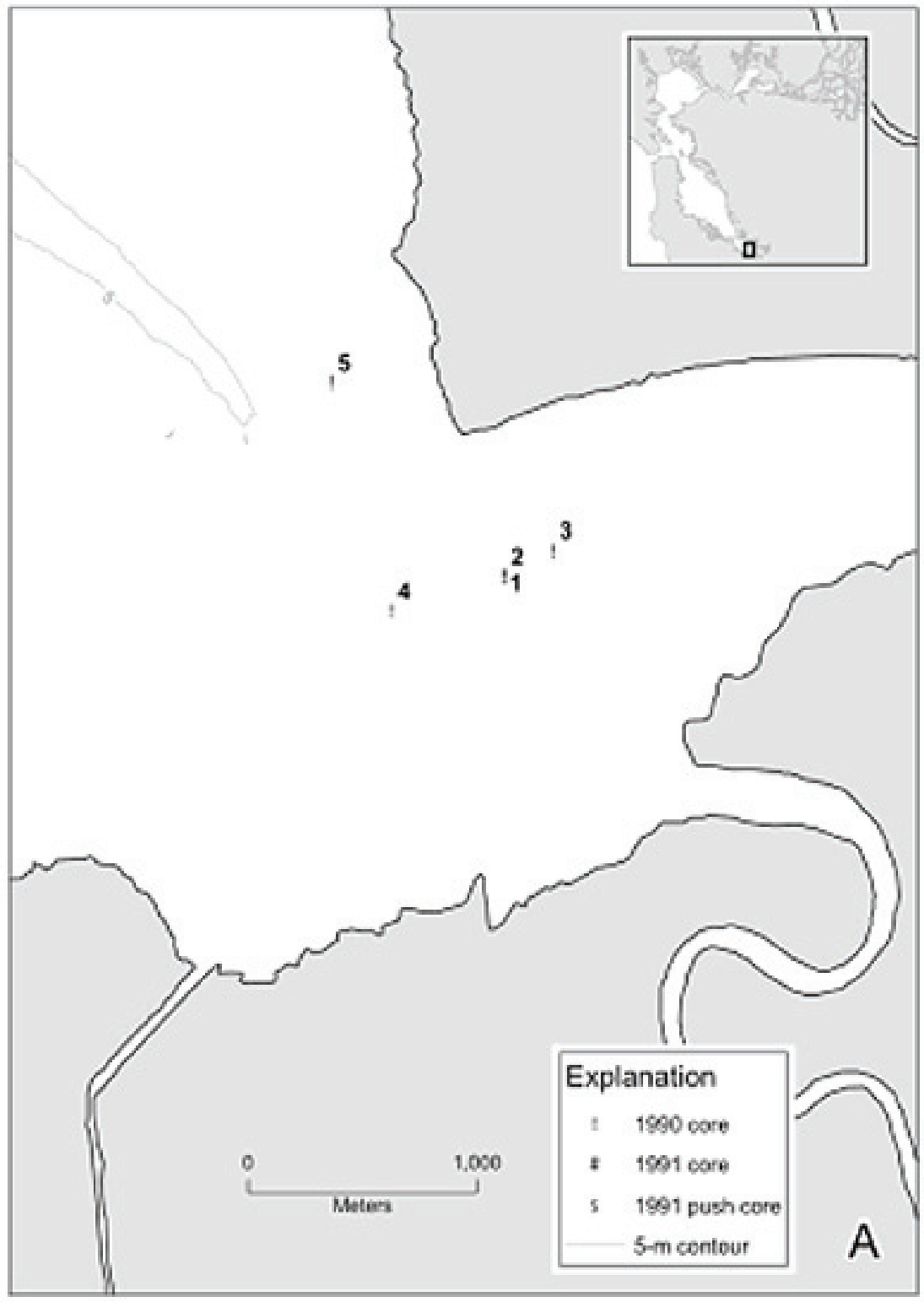

Figure 2 Detailed map of Area A showing locations of cores collected in South San Francisco Bay between the confluences of Guadalupe River and Coyote Creek. 


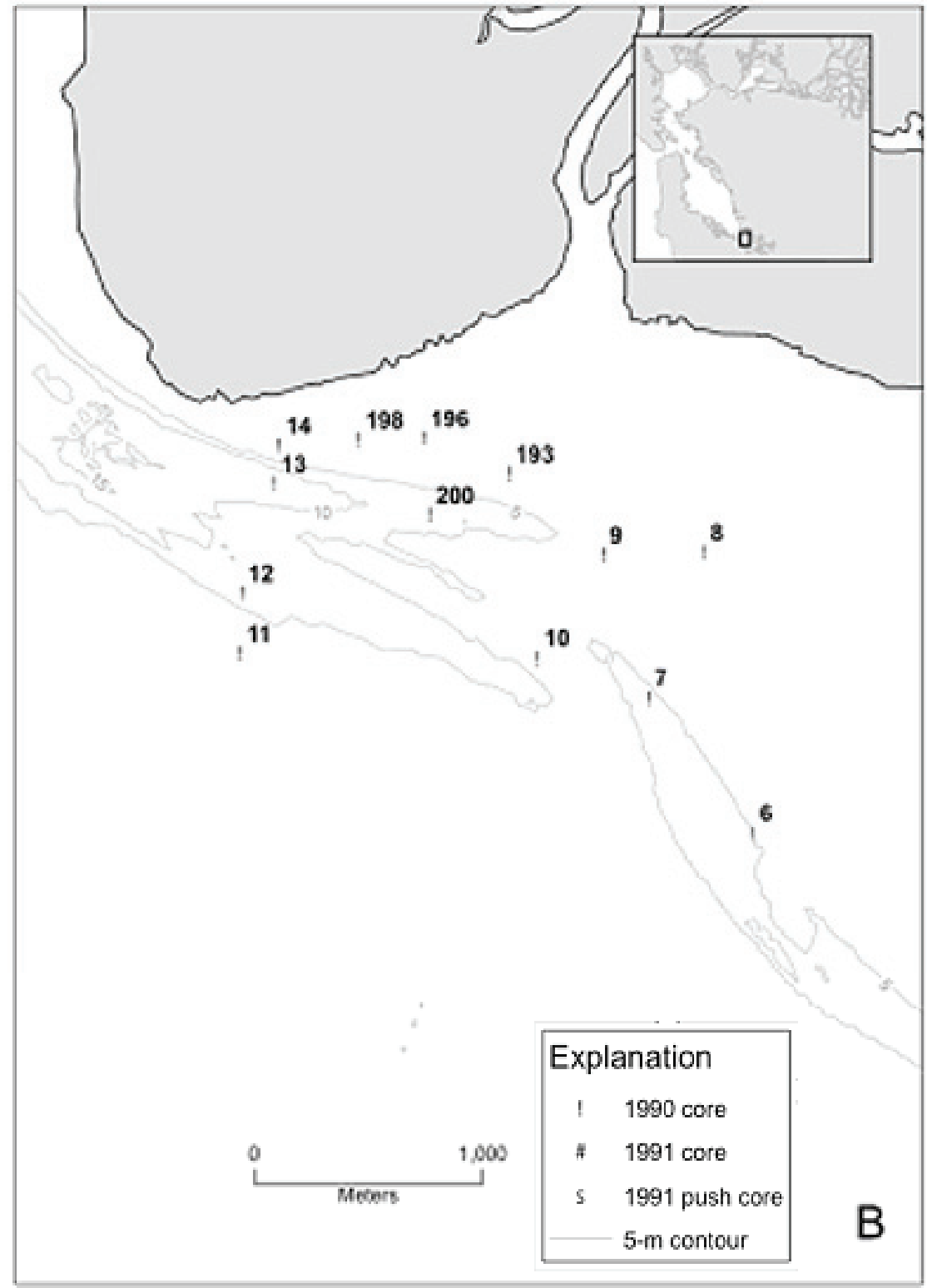

Figure 3 Detailed map of Area B showing locations of cores collected South of the Dumbarton Bridge near the mouth of Newark Slough. 


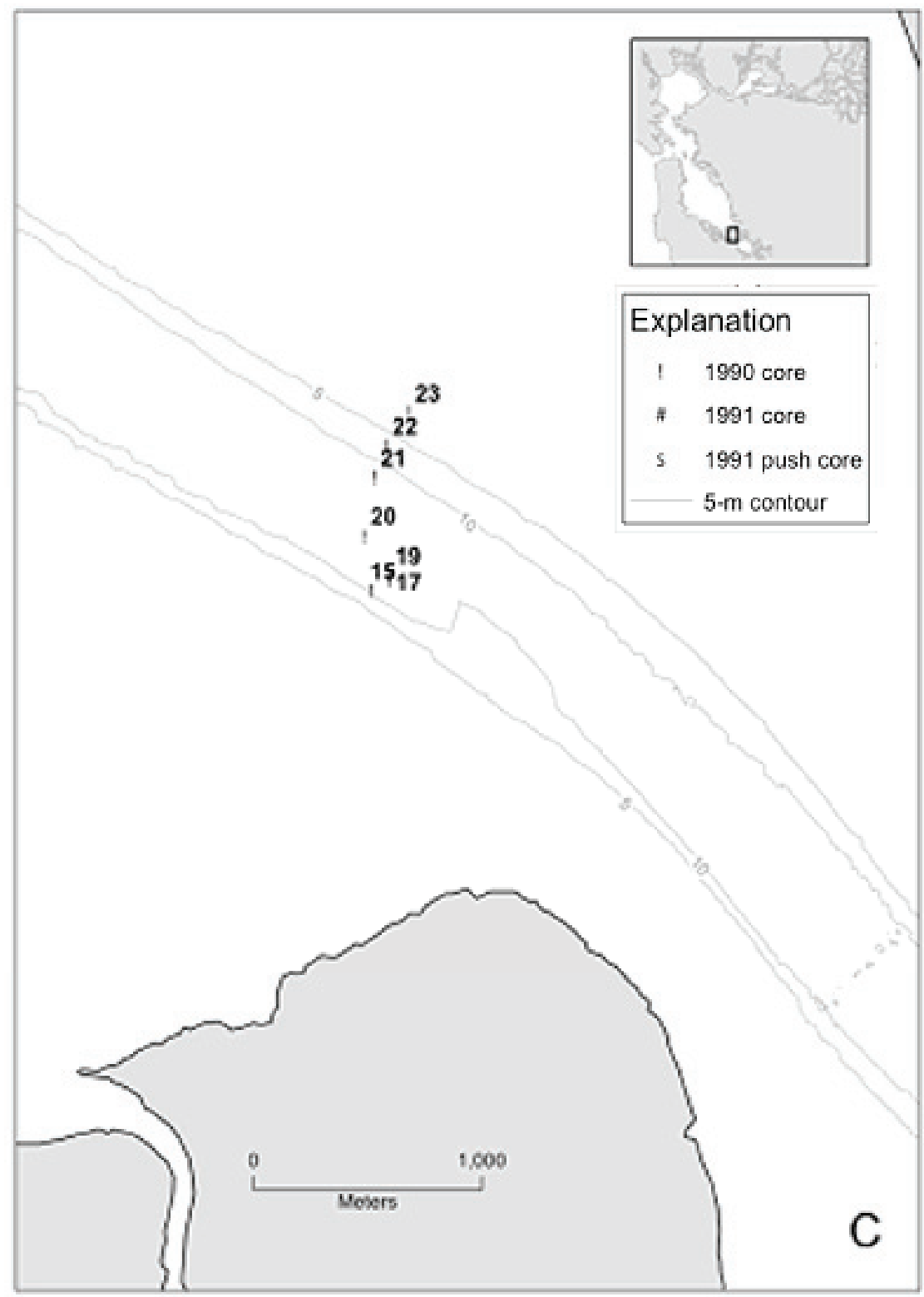

Figure 4 Detailed map of Area $\mathrm{C}$ showing locations of cores collected north of the Dumbarton Bridge across the main channel. 


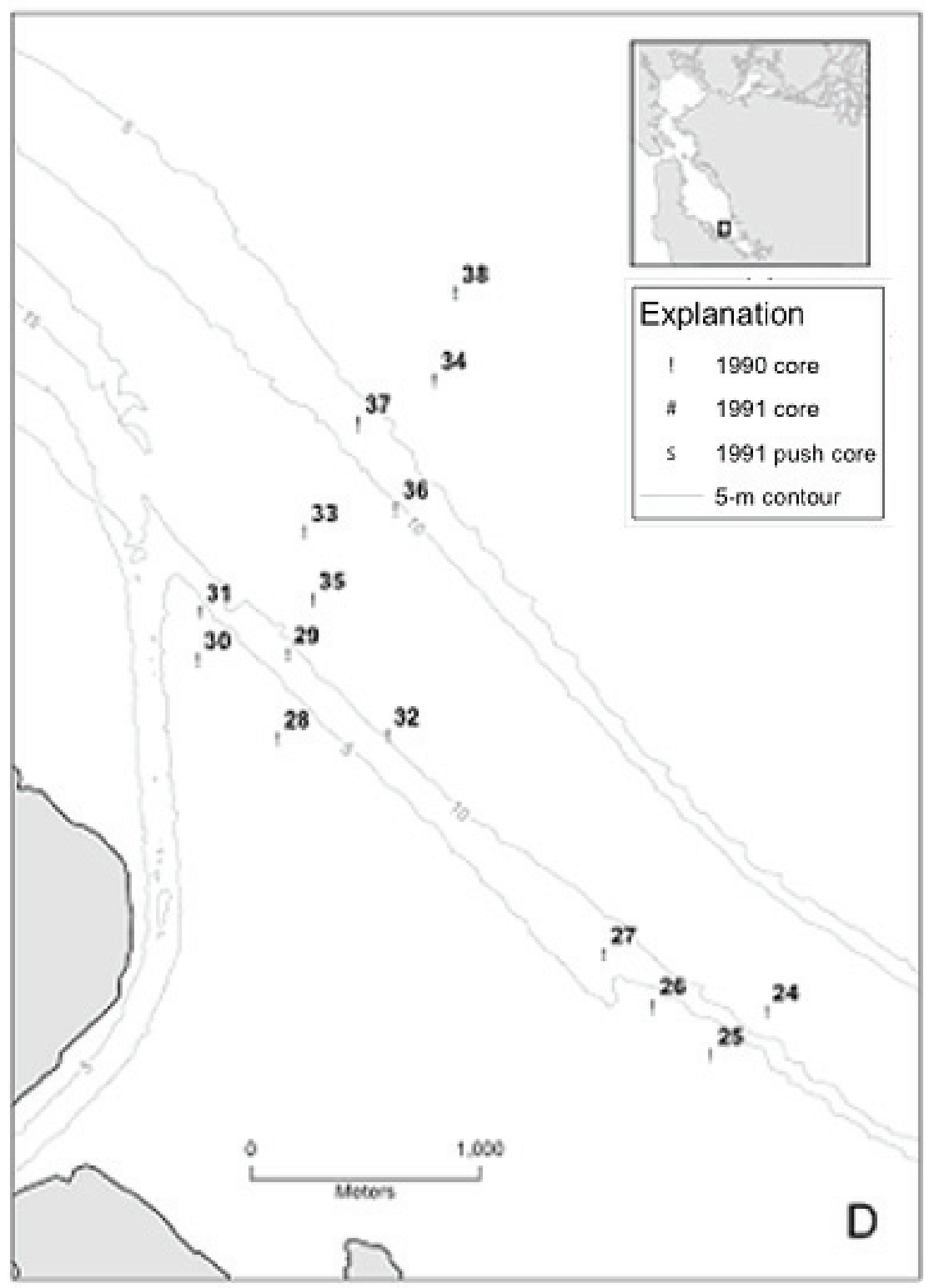

Figure 5 Detailed map of Area D showing locations of cores collected north and south of the mouth of Redwood Creek, across the main channel and subtidal flats. 


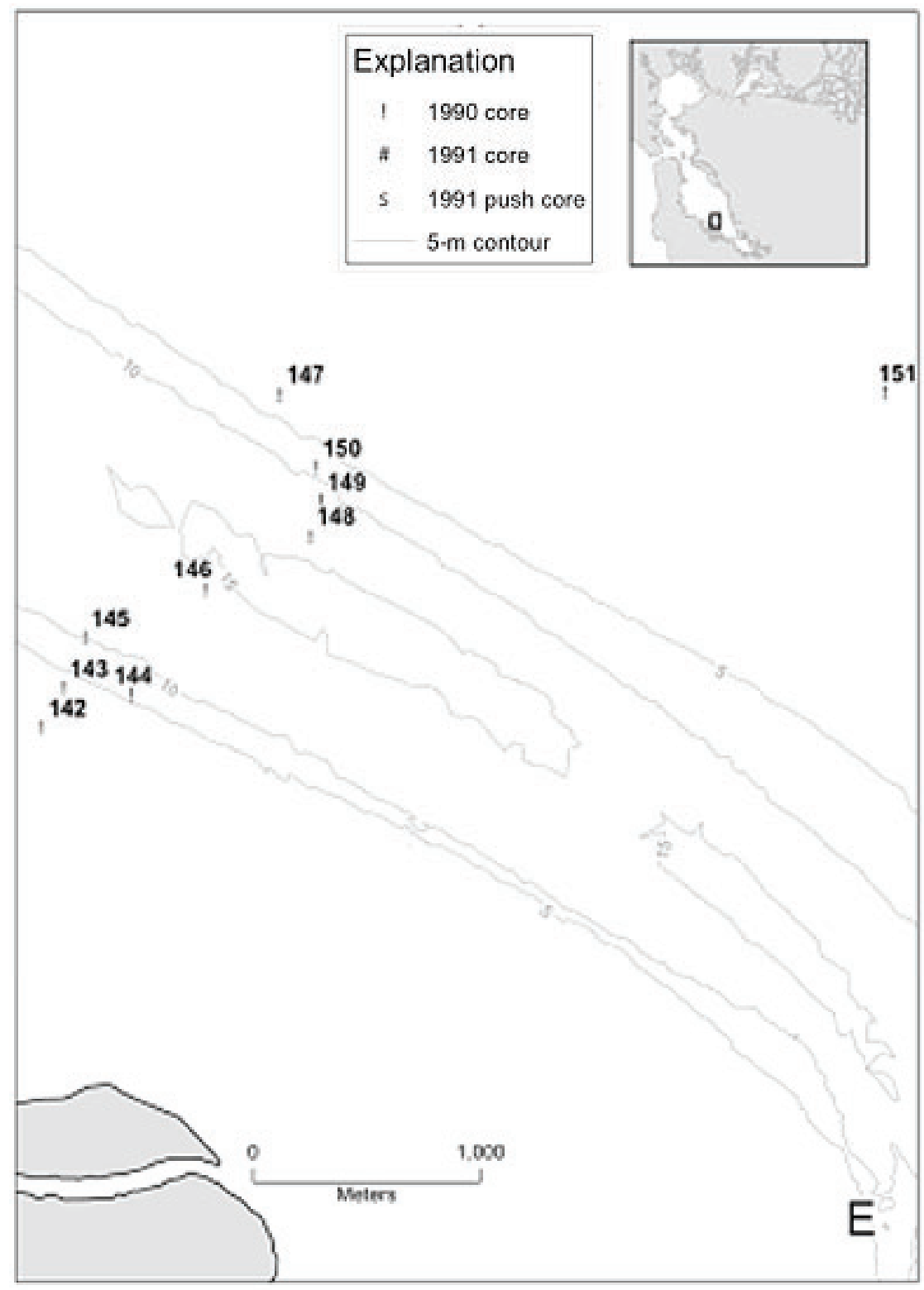

Figure 6 Detailed map of Area E showing locations of cores collected south of the San Mateo Bridge across the main channel. 


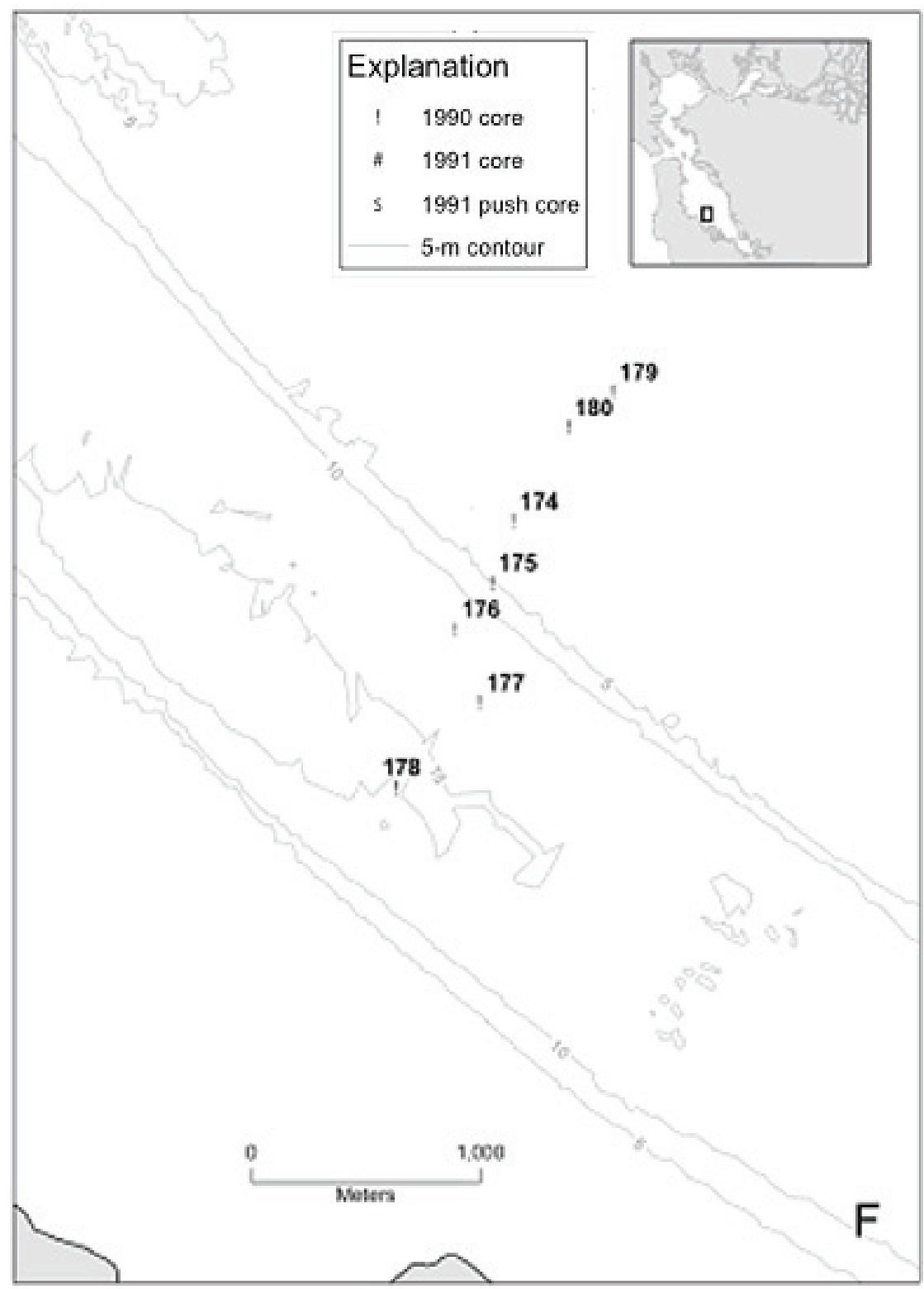

Figure 7 Detailed map of Area F showing locations of cores collected in South San Francisco Bay across the main channel and subtidal flats. 


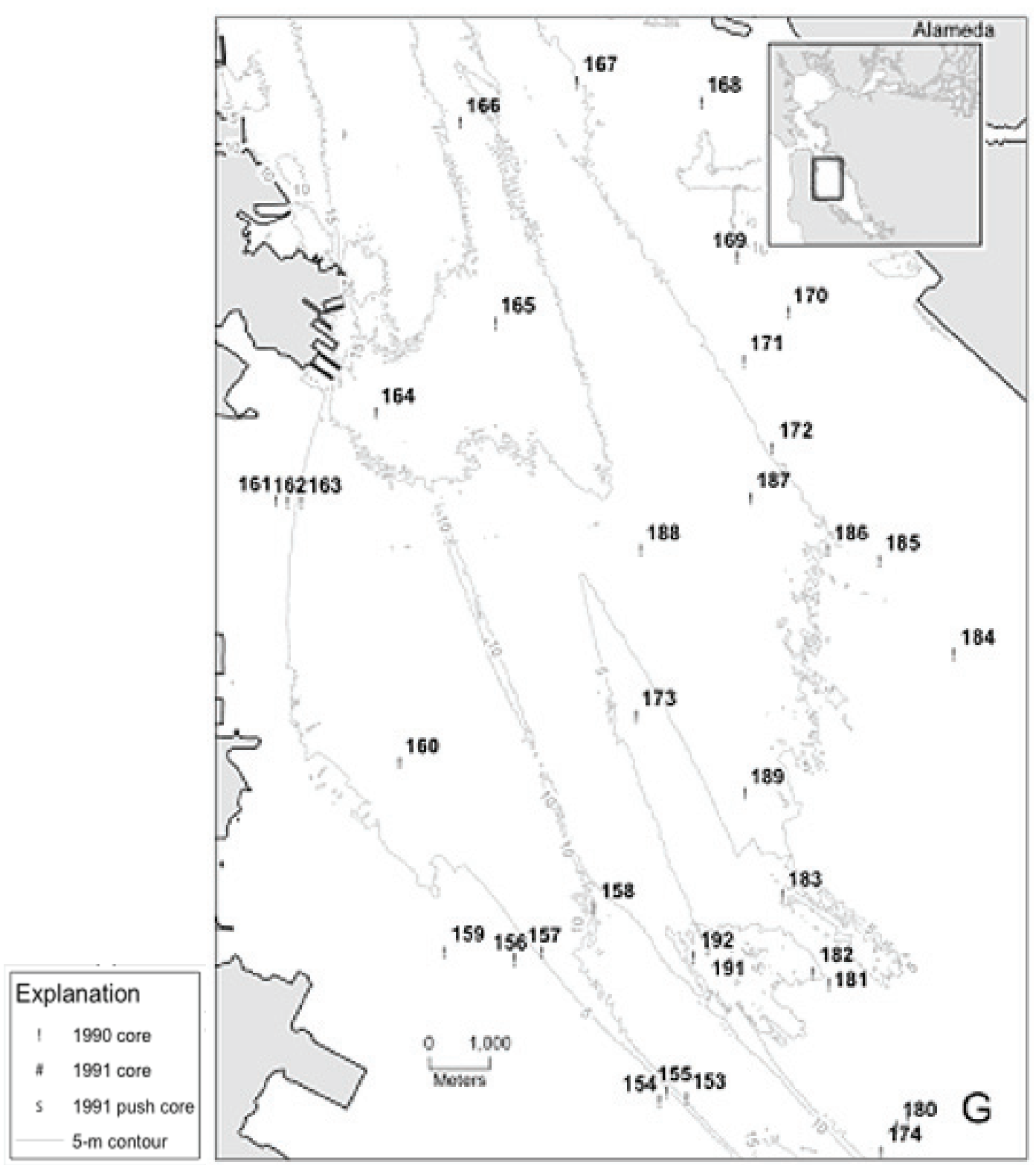

Figure 8 Detailed map of Area G showing locations of cores collected in South-central San Francisco Bay between the San Francisco-Oakland Bay Bridge and San Mateo Bridge. 


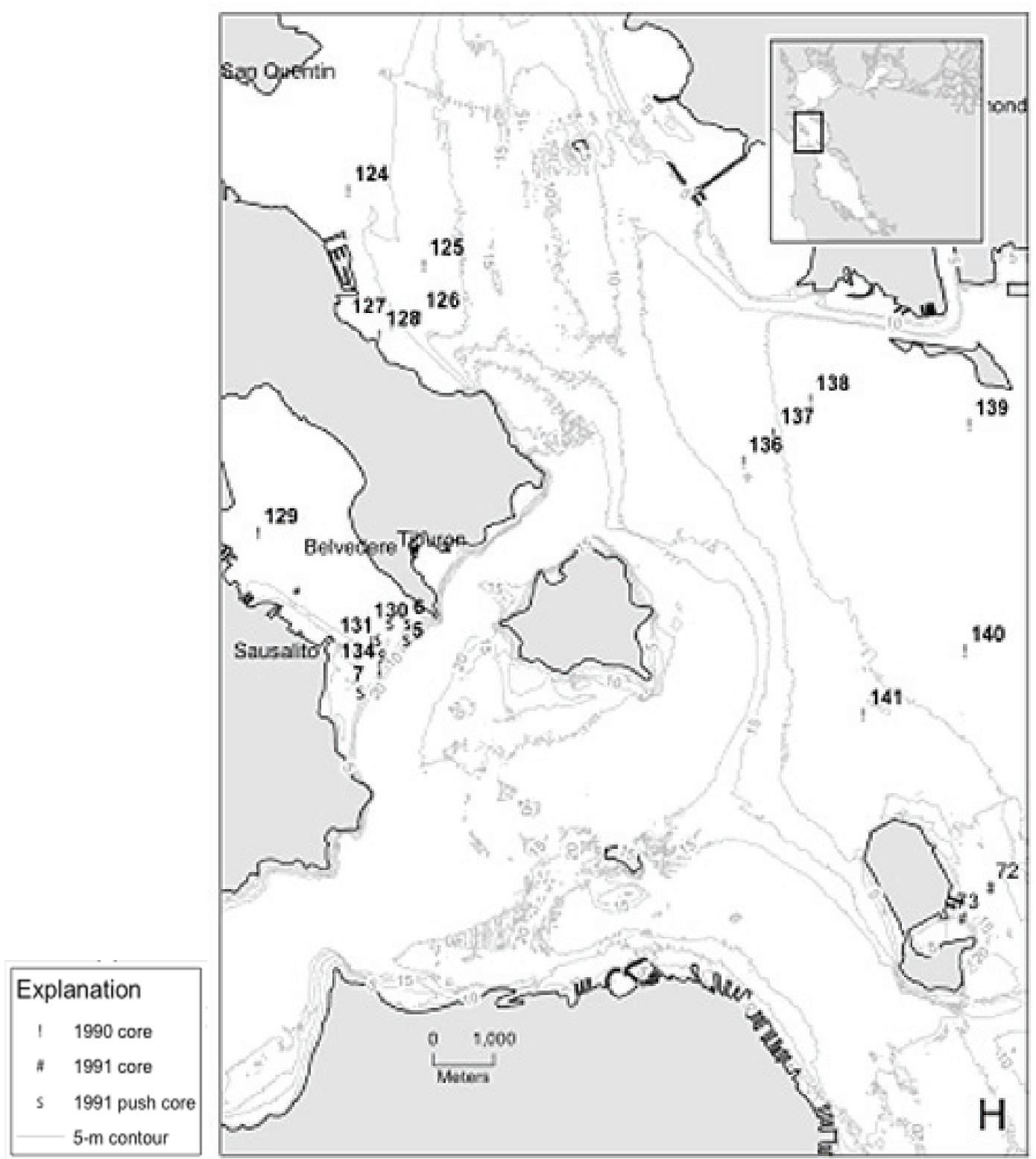

Figure 9 Detailed map of Area H showing locations of cores collected in central San Francisco Bay. Note detailed area I at the mouth of Richardson Bay. 


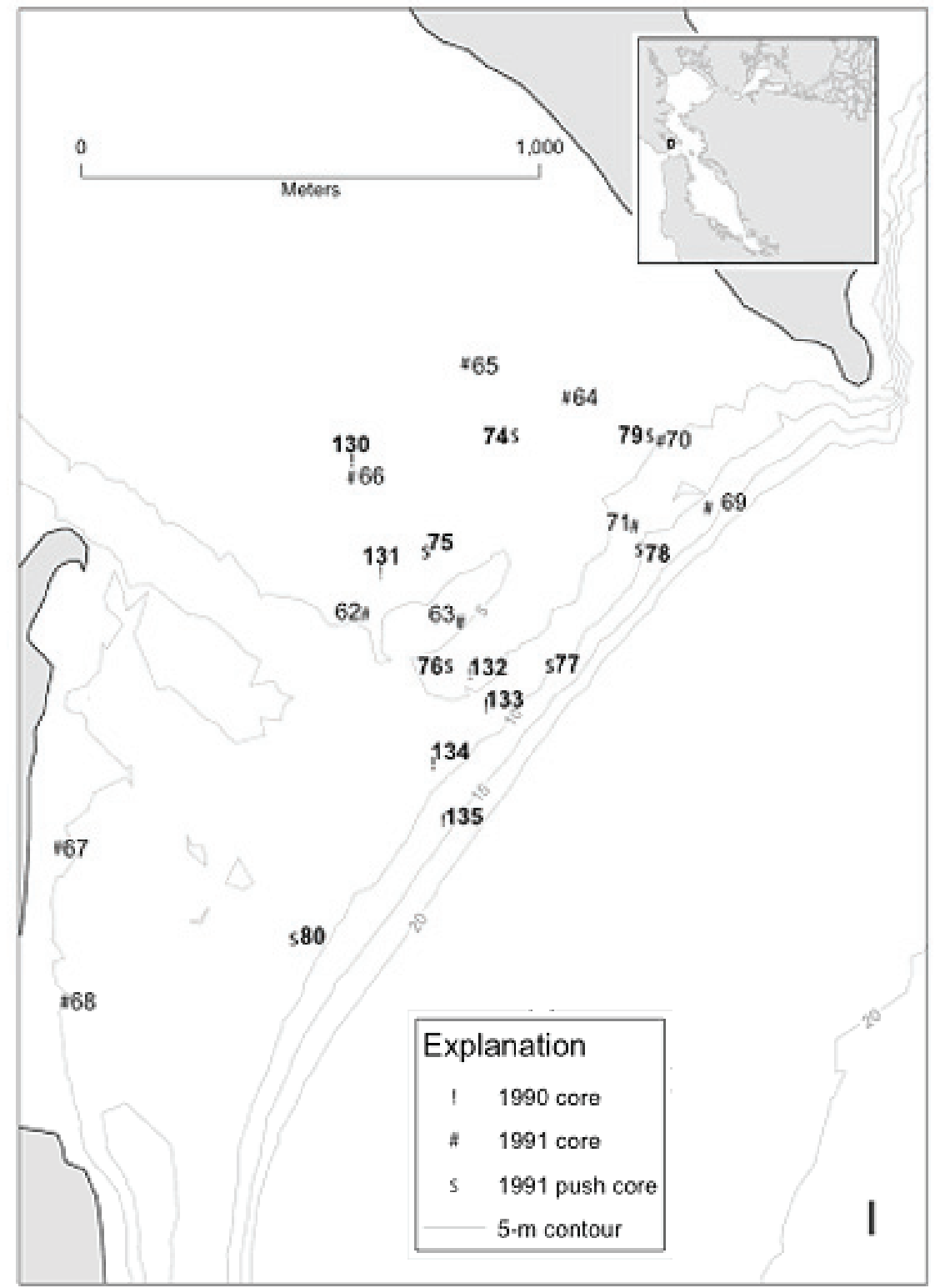

Figure 10 Detailed map of Area I showing locations of cores collected at the mouth of Richardson Bay on the slope of the main tidal channel and upper sub tidal flats. 


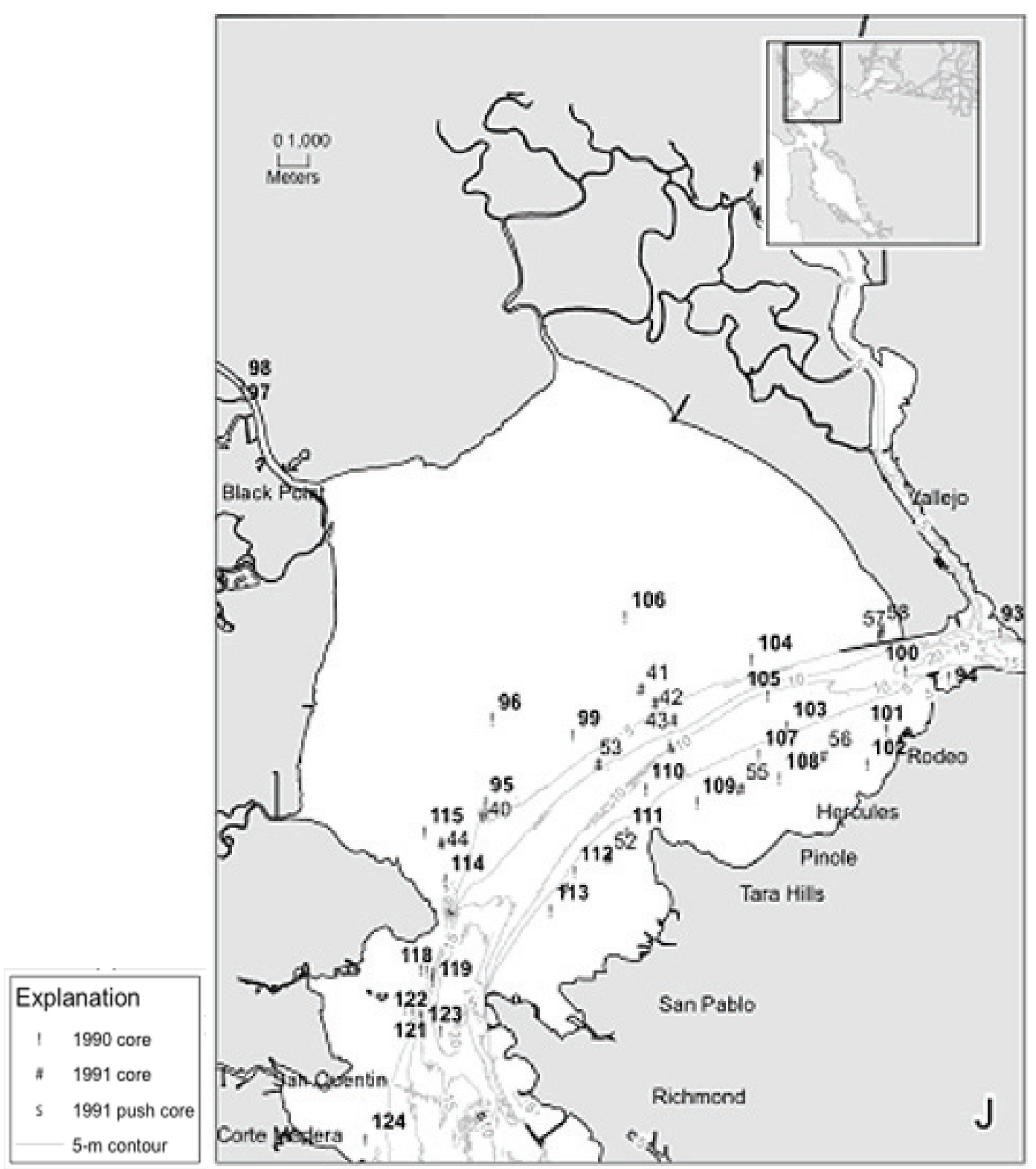

Figure 12 Detailed map of Area J showing locations of cores collected in San Rafael and San Pablo Bays, the Petaluma River to the Carquinez Bridge. 


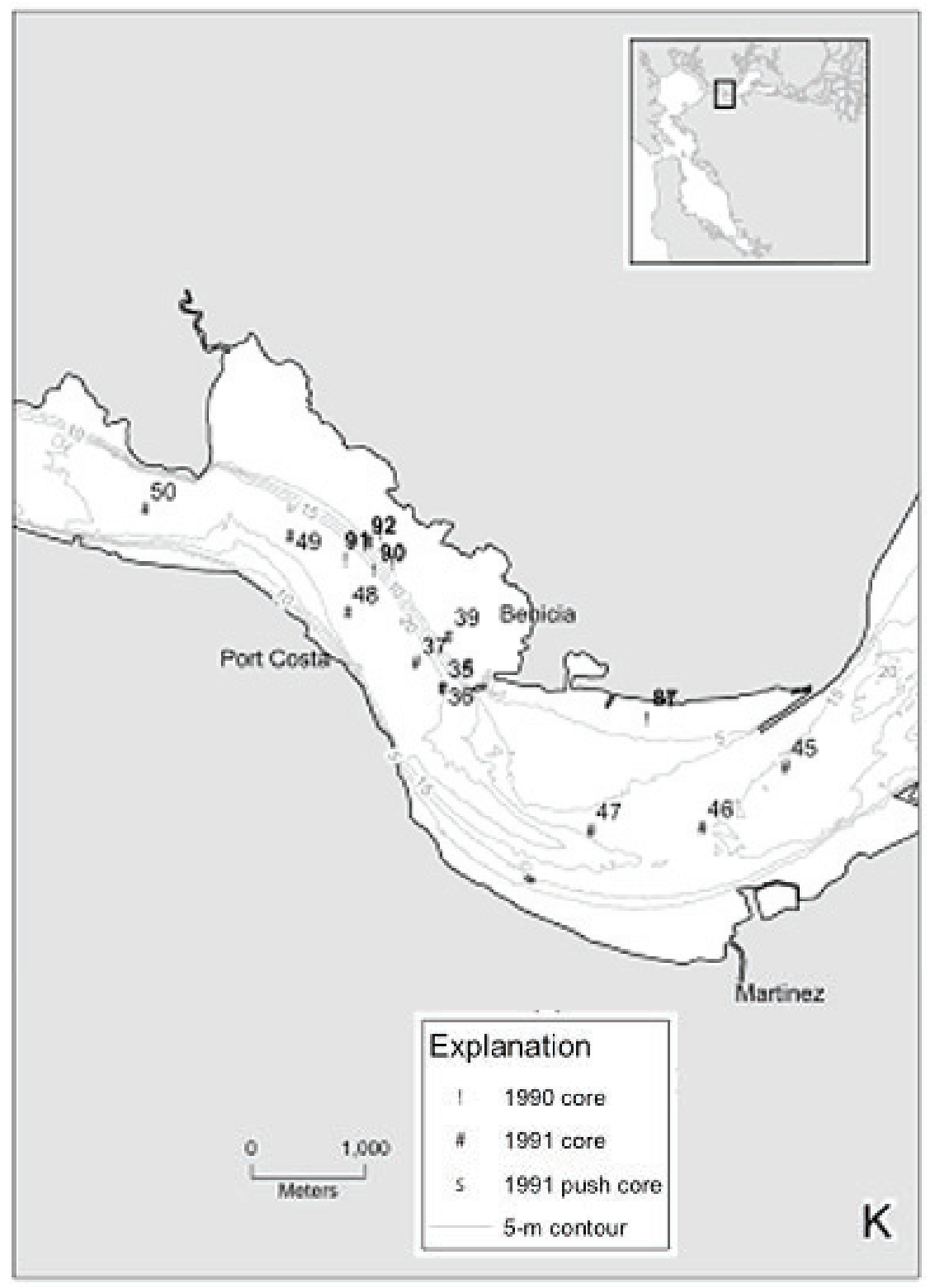

Figure 13 Detailed map of Area K showing locations of cores collected in Carquinez Strait. Note detailed map adjacent to Southampton Bay. 


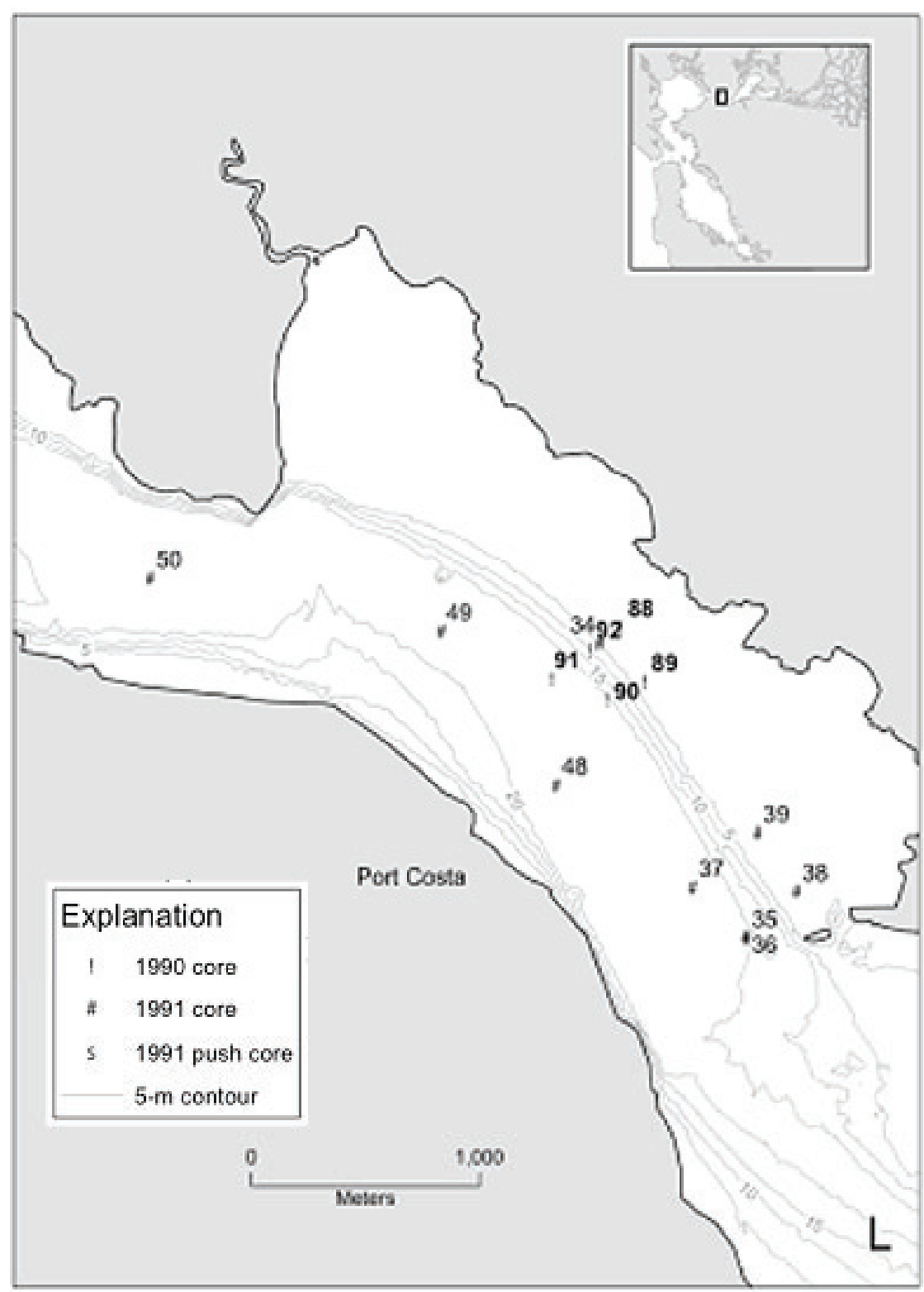

Figure 14 Detailed map of Area L showing locations of cores collected between Dillon Point and the city wharf at Benicia. 


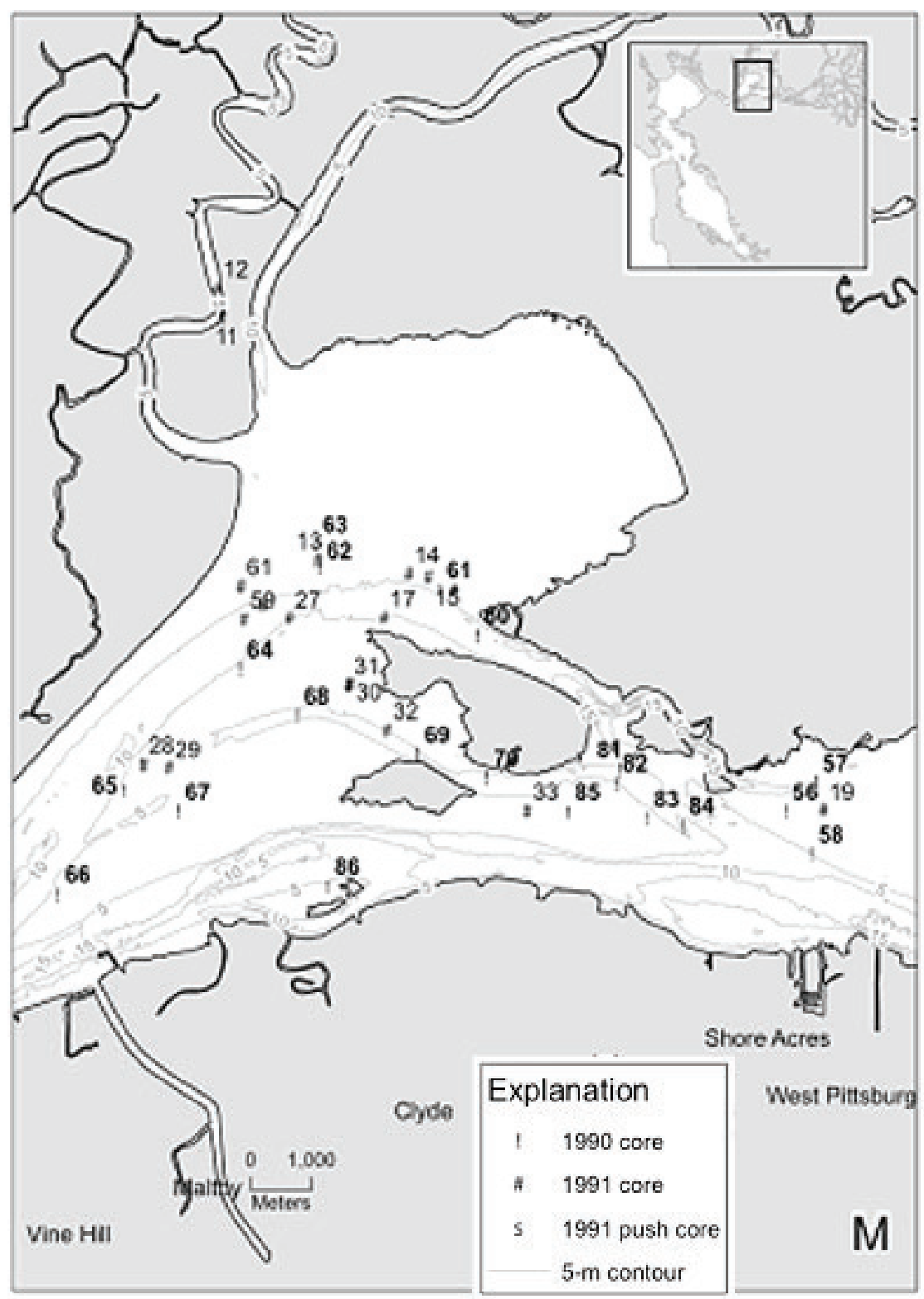

Figure 15 Detailed map of Area M showing locations of cores collected in Suisun Bay east of the Martinez-Benicia Bridge and Chipps Island, and in Suisun Slough. Note Ryer and Roe Islands in the center of the figure. 


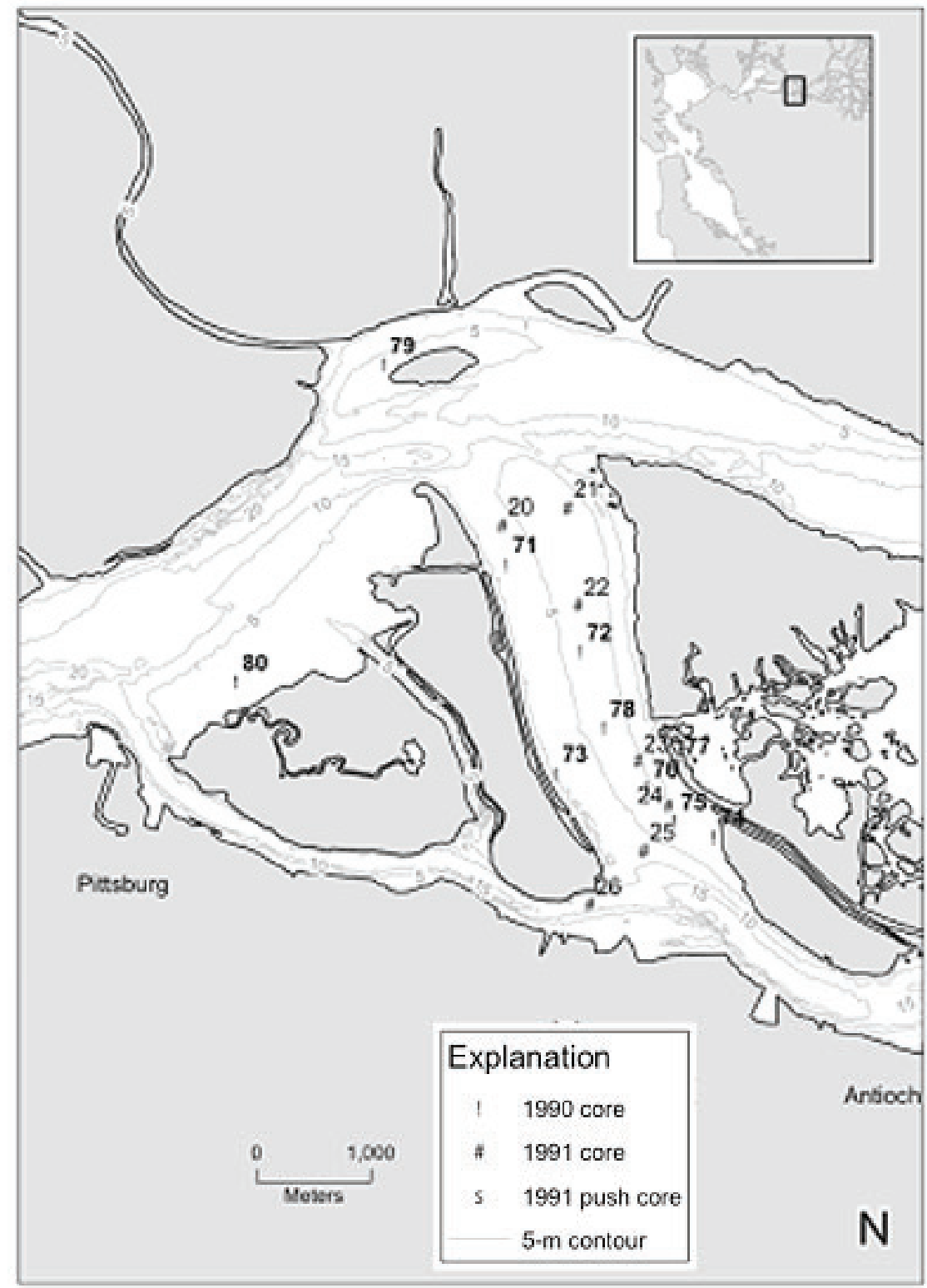

Figure 16 Detailed map of Area N showing locations of cores collected along the confluence of the Sacramento and San Joaquin Rivers. Note from left center to right center of the figure; Browns, Winter and Sherman Islands. 


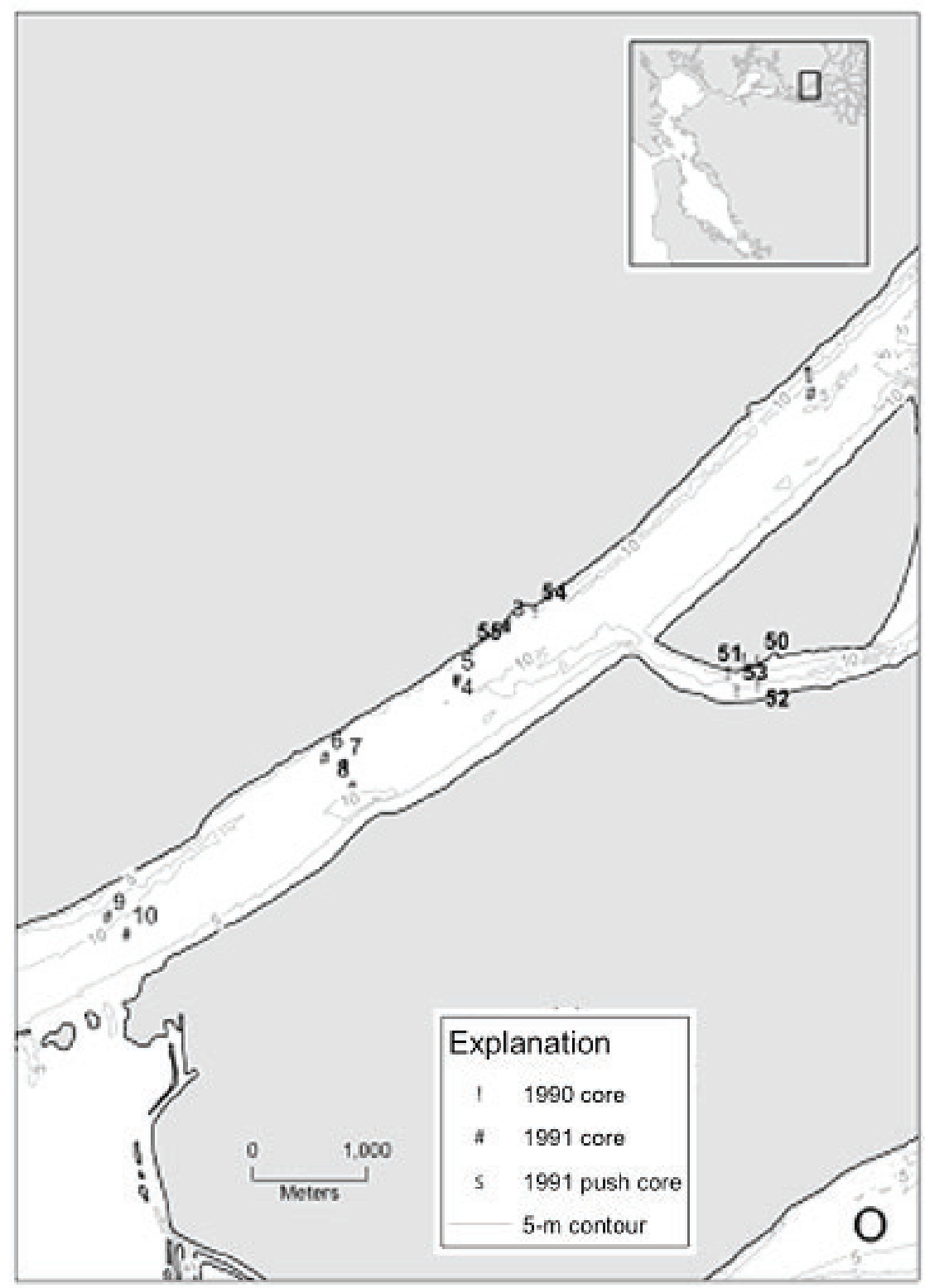

Figure 17 Detailed map of Area O showing locations of cores collected along the Sacramento River from Sherman to Decker Islands. 


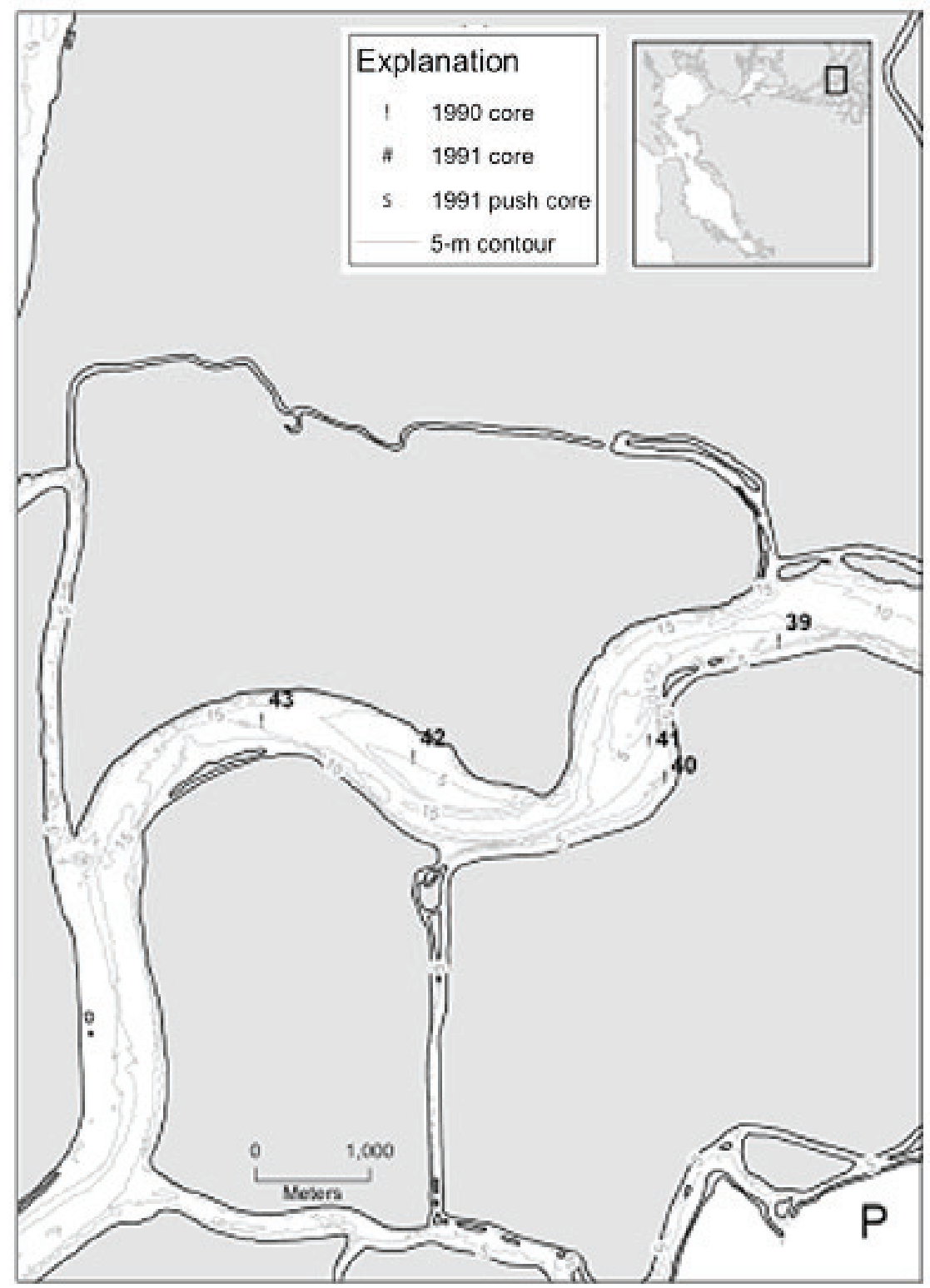

Figure 18 Detailed map of Area P showing locations of cores collected along the San Joaquin River between Three Mile Slough and Seven mile Slough. 


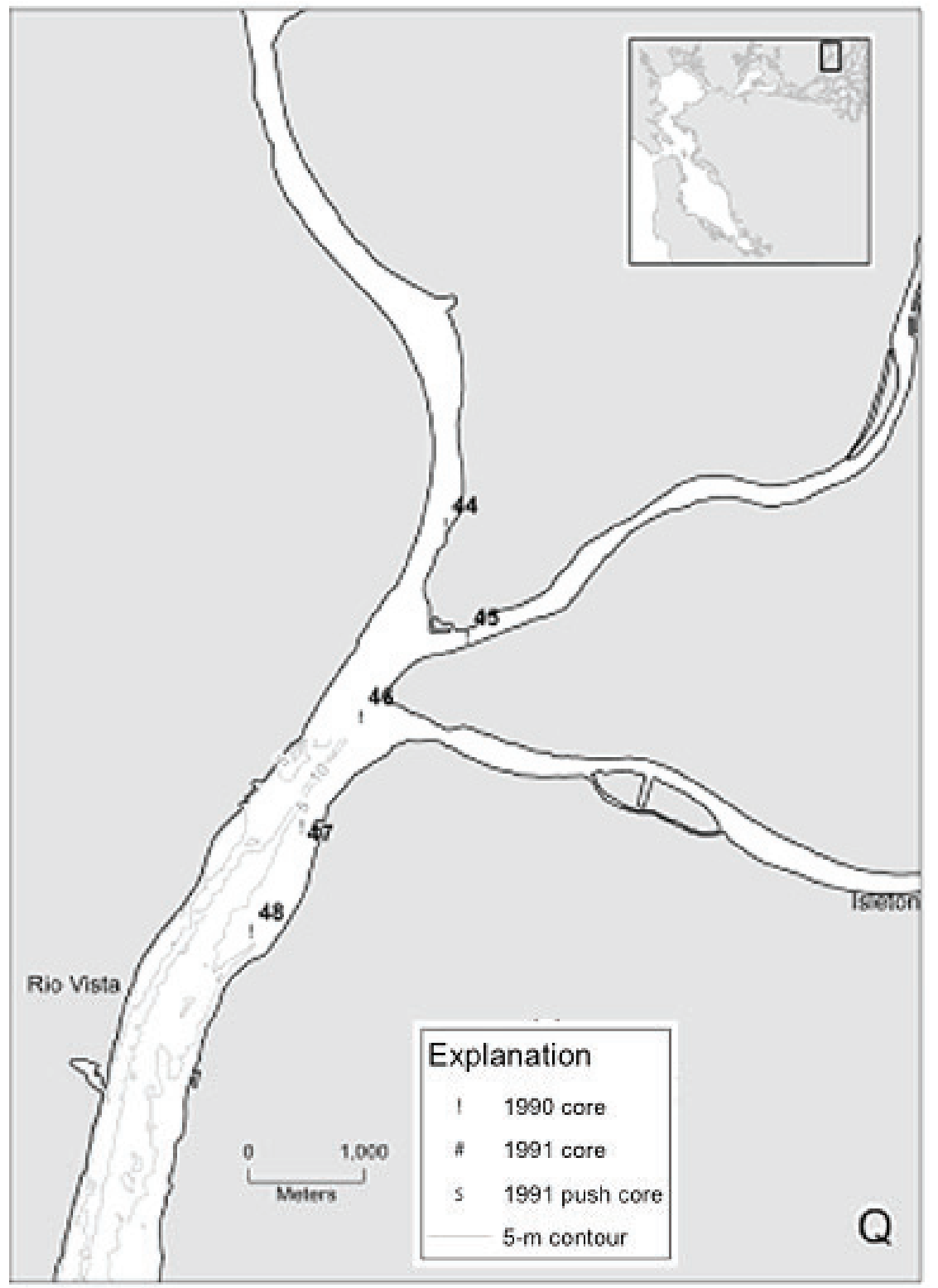

Figure 19 Detailed map of Area Q showing locations of cores collected on the Sacramento River northeast of Rio Vista to Steamboat and Cache Sloughs. 
CORE TABLES 


\begin{tabular}{|c|c|c|c|c|c|c|c|}
\hline $\begin{array}{l}\text { Мap } \\
\#\end{array}$ & $\begin{array}{l}\text { Core } \\
\text { Number }\end{array}$ & Setting & $\begin{array}{c}\text { Lat. } \\
\text { Long. }\end{array}$ & $\begin{array}{l}\text { Water } \\
\text { Depth (m) }\end{array}$ & $\begin{array}{l}\text { Type } \\
\text { Core (G) } \\
\text { (B) }\end{array}$ & $\begin{array}{l}\text { Core } \\
\text { length } \\
\text { (m) }\end{array}$ & General Description \\
\hline
\end{tabular}

\section{J-1-90-SF \\ 1990 Cores \\ Map A \& B}

South San Francisco Bay, South of the Dumbarton Bridge

\begin{tabular}{|c|c|c|c|c|c|c|c|}
\hline 1 & $1-\mathrm{G}-23$ & Coyote Crk. mouth N. Bank & $\begin{array}{r}37^{\circ} 27.65^{\prime} \\
-122^{\circ} 2.83^{\prime} \\
\end{array}$ & 4.4 & $\begin{array}{l}200 \mathrm{lb} 3.0 \mathrm{~m} \\
\text { barrel }(\mathrm{G})\end{array}$ & 1.34 & faintly stiff mud \\
\hline 2 & $2-\mathrm{G}-23$ & Coyote Crk. mouth up slope & $\begin{array}{r}37^{\circ} 27.65^{\prime} \\
-122^{\circ} 2.83^{\prime}\end{array}$ & 2 & $\begin{array}{l}200 \mathrm{lb} 3.0 \mathrm{~m} \\
\text { barrel }(\mathrm{G})\end{array}$ & 1.19 & $\begin{array}{l}\text { Less cohesive mud at the } \\
\text { surface }\end{array}$ \\
\hline 3 & $3-\mathrm{G}-23$ & $\begin{array}{l}\text { Coyote Crk mouth E. of \#2 N. } \\
\text { bank }\end{array}$ & $\begin{array}{r}37^{\circ} 27.70^{\prime} \\
-122^{\circ} 2.68^{\prime} \\
\end{array}$ & 2 & $\begin{array}{l}3.0 \mathrm{~m} \text { barrel } \\
(\mathrm{G})\end{array}$ & 2.10 & $\begin{array}{l}\text { probable compaction shelly } \\
\text { mud }\end{array}$ \\
\hline 4 & $4-\mathrm{G}-23$ & $\begin{array}{l}\text { Btwn Coyote Crk \& } \\
\text { Guadalupe Slough in the } \\
\text { Thalweg }\end{array}$ & $\begin{array}{r}37^{\circ} 27.57^{\prime} \\
-122^{\circ} 3.17^{\prime}\end{array}$ & 3.25 & $\begin{array}{l}3.0 \mathrm{~m} \text { barrel } \\
(\mathrm{G})\end{array}$ & 1.50 & lower slowly, shell hash at top \\
\hline 5 & $5-G-23$ & $\begin{array}{l}\text { Upper subtidal flats NW of } \\
\text { Calaveras Pt. }\end{array}$ & $\begin{array}{r}37^{\circ} 28.10^{\prime} \\
-122^{\circ} 3.34^{\prime} \\
\end{array}$ & 2.7 & $\begin{array}{l}3.0 \mathrm{~m} \text { barrel } \\
(\mathrm{G})\end{array}$ & 1.53 & lower slowly, shell hash at top \\
\hline 6 & $6-G-23$ & $\begin{array}{l}\text { Channel Slope N. of Marker } \\
\# 17\end{array}$ & $\begin{array}{r}37^{\circ} 28.92^{\prime} \\
-122^{\circ} 4.58^{\prime} \\
\end{array}$ & 7.5 & $\begin{array}{l}3.0 \mathrm{~m} \text { barrel } \\
(\mathrm{G})\end{array}$ & 1.37 & $\begin{array}{l}\text { free fall, some shelly material } \\
\text { at the top }\end{array}$ \\
\hline 7 & 7-G-23 & $\begin{array}{l}\text { South of Marker \#17 on shoal } \\
\text { area }\end{array}$ & $\begin{array}{r}37^{\circ} 29.23^{\prime} \\
-122^{\circ} 4.89^{\prime} \\
\end{array}$ & 5 & $\begin{array}{l}3.0 \mathrm{~m} \text { barrel } \\
(\mathrm{G})\end{array}$ & 1.06 & $\begin{array}{l}\text { free fall, shell lag at top and } \\
\text { bottom }\end{array}$ \\
\hline 8 & $1-\mathrm{G}-24$ & $\begin{array}{l}\text { Mouth of Mowry Slough N. } \\
\text { side }\end{array}$ & $\begin{array}{r}37^{\circ} 29.57^{\prime} \\
-122^{\circ} 4.72^{\prime}\end{array}$ & 3.75 & $\begin{array}{l}3.0 \mathrm{~m} \text { barrel } \\
(\mathrm{G})\end{array}$ & 2.06 & $\begin{array}{l}\text { compacting sediment buried } \\
\text { head layer of coarse material } \\
\cong 50 \mathrm{~cm} \text { below the top }\end{array}$ \\
\hline 9 & $2-\mathrm{G}-24$ & $\begin{array}{l}\text { Down slope from \#8 at mouth } \\
\text { of Mowry Slough NE Mkr \#16 }\end{array}$ & $\begin{array}{r}37^{\circ} 29.57^{\prime} \\
-122^{\circ} 5.02^{\prime} \\
\end{array}$ & 6 & $\begin{array}{l}1.75 \mathrm{~m} \text { barrel } \\
(\mathrm{G})\end{array}$ & 2.03 & top disturbed free fall \\
\hline 10 & $3-G-24$ & $\begin{array}{l}\text { West of Marker \#16 on shoal } \\
\text { area across from mouth of } \\
\text { Newark Slough }\end{array}$ & $\begin{array}{r}37^{\circ} 29.33^{\prime} \\
-122^{\circ} 5.21^{\prime}\end{array}$ & 6.25 & $\begin{array}{l}3.0 \mathrm{~m} \text { barrel } \\
(\mathrm{G})\end{array}$ & 1.97 & coarse layer $\cong 25 \mathrm{~cm}$ from top \\
\hline 11 & $4-\mathrm{G}-24$ & $\begin{array}{l}\text { West of Marker \#16 across } \\
\text { channel from Newark Slough } \\
\text { at top of channel bank }\end{array}$ & $\begin{array}{r}37^{\circ} 29.35^{\prime} \\
-122^{\circ} 6.10^{\prime}\end{array}$ & 4.3 & $\begin{array}{l}3.0 \mathrm{~m} \text { barrel } \\
(\mathrm{G})\end{array}$ & 2.19 & $\begin{array}{l}\text { top disturbed worm tubes near } \\
\text { the top }\end{array}$ \\
\hline 12 & $5-\mathrm{G}-24$ & Thalweg down slope from $\# 11$ & $\begin{array}{r}37^{\circ} 29.49^{\prime} \\
-122^{\circ} 6.09^{\prime}\end{array}$ & 8.3 & $\begin{array}{l}3.0 \mathrm{~m} \text { barrel } \\
(\mathrm{G})\end{array}$ & 1.72 & $\begin{array}{l}\text { shell lag and stiff mud at the } \\
\text { bottom, shell fragments on top }\end{array}$ \\
\hline
\end{tabular}




\begin{tabular}{|c|c|c|c|c|c|c|c|}
\hline $\begin{array}{c}\text { Мap } \\
\#\end{array}$ & $\begin{array}{l}\text { Core } \\
\text { Number }\end{array}$ & Setting & $\begin{array}{l}\text { Lat. } \\
\text { Long. }\end{array}$ & $\begin{array}{l}\text { Water } \\
\text { Depth (m) }\end{array}$ & $\begin{array}{l}\text { Type } \\
\text { Core (G) } \\
\text { (B) }\end{array}$ & $\begin{array}{l}\text { Core } \\
\text { length } \\
\text { (m) }\end{array}$ & General Description \\
\hline
\end{tabular}

\begin{tabular}{|c|c|c|c|c|c|c|c|}
\hline 13 & $6-\mathrm{G}-24$ & $\begin{array}{l}\text { On channel slope near mouth } \\
\text { of Newark Slough }\end{array}$ & $\begin{array}{r}37^{\circ} 29.74^{\prime} \\
-122^{\circ} 5.99^{\prime}\end{array}$ & 5 & $\begin{array}{l}3.0 \mathrm{~m} \text { barrel } \\
\text { (G) }\end{array}$ & 1.67 & faintly stiff mud at bottom \\
\hline 14 & $7-B C-24$ & $\begin{array}{l}\text { Mouth of Newark Slough } \\
\text { down slope from \#13 }\end{array}$ & $\begin{array}{r}37^{\circ} 29.83^{\prime} \\
-122^{\circ} 5.97^{\prime}\end{array}$ & 5.5 & (B) box core & & $\begin{array}{l}\text { had } 3 \text { to } 4 \mathrm{~cm} \text { of mud above } \\
\text { box-disturbed upper layer. }\end{array}$ \\
\hline
\end{tabular}

\begin{tabular}{|c|c|c|c|c|c|c|c|}
\hline \multicolumn{8}{|c|}{$\begin{array}{l}\text { J-1-90-SF } \\
\text { South San Francisco Bay, Between the San Mateo and Dumbarton Bridge } \\
\text { Map B, C, \& D }\end{array}$} \\
\hline 15 & $8-\mathrm{BC}-24$ & $\begin{array}{l}\text { S. Bay just N. of Dumbarton } \\
\text { Bridge, } \\
\text { on west side of the main } \\
\text { channel near top of channel } \\
\text { bank }\end{array}$ & $\begin{array}{r}37^{\circ} 31.18^{\prime} \\
-122^{\circ} 8.56^{\prime}\end{array}$ & 5.6 & (B) & & $\begin{array}{l}\text { shell at the top worm tubes } \\
\text { w/worms }\end{array}$ \\
\hline 16 & $9-\mathrm{BC}-24$ & $\begin{array}{l}\text { Same area as \#15 down slope } \\
\text { on same bank }\end{array}$ & $\begin{array}{r}37^{\circ} 31.18^{\prime} \\
-122^{\circ} 8.56^{\prime}\end{array}$ & 8.25 & (B) & & $\begin{array}{l}\text { big worms and tubes cores are } \\
\text { aligned to each other with a } \\
\text { line to long end of box core }\end{array}$ \\
\hline 17 & $10-\mathrm{G}-24$ & $\begin{array}{l}\text { Same area as } \# 15 \& 16 \text { down } \\
\text { slope near the thalweg }\end{array}$ & $\begin{array}{r}37^{\circ} 31.20^{\prime} \\
-122^{\circ} 8.51^{\prime}\end{array}$ & 9 & $\begin{array}{l}3.0 \mathrm{~m} \text { barrel } \\
(\mathrm{G})\end{array}$ & .40 & \\
\hline 18 & $11-\mathrm{G}-24$ & $\begin{array}{l}\text { Same area as \#16 \& } 17 \text { in the } \\
\text { thalweg }\end{array}$ & $\begin{array}{r}37^{\circ} 31.20^{\prime} \\
-122^{\circ} 8.51^{\prime}\end{array}$ & 9 & $\begin{array}{l}3.0 \mathrm{~m} \text { barrel } \\
(\mathrm{G})\end{array}$ & 1.20 & $\begin{array}{l}\text { free fall, sand, shell bed } \\
\text { oysters in core catcher }\end{array}$ \\
\hline 19 & $12-\mathrm{G}-24$ & $\begin{array}{l}\text { North side of channel in line } \\
\text { with } \# 18 \text { along the thalweg }\end{array}$ & $\begin{array}{r}37^{\circ} 31.22^{\prime} \\
-122^{\circ} 8.51^{\prime} \\
\end{array}$ & 11 & $\begin{array}{l}3.0 \mathrm{~m} \text { barrel } \\
(\mathrm{G})\end{array}$ & 1.01 & free fall \\
\hline 20 & $1-\mathrm{G}-25$ & North of \#19 along thalweg & $\begin{array}{r}37^{\circ} 31.31^{\prime} \\
-122^{\circ} 8.58^{\prime}\end{array}$ & 18 & $\begin{array}{l}2.5 \mathrm{~m} \text { barrel } \\
(\mathrm{G})\end{array}$ & 1.34 & $\begin{array}{l}\text { Oyster shells in core catcher, } \\
\text { shell with sandy silt on bottom, } \\
\text { top has shells, tube worms, silt }\end{array}$ \\
\hline 21 & $2-\mathrm{G}-25$ & $\begin{array}{l}\text { Along the break in slope in } \\
\text { line with } \# 20\end{array}$ & $\begin{array}{r}37^{\circ} 31.45^{\prime} \\
-122^{\circ} 8.55^{\prime} \\
\end{array}$ & 14.7 & $\begin{array}{l}2.5 \mathrm{~m} \text { barrel } \\
(\mathrm{G})\end{array}$ & .65 & shells and sand at the bottom \\
\hline 22 & $3-\mathrm{G}-25$ & $\begin{array}{l}\text { Up slope from \# } 21 \text { north side } \\
\text { of main channel }\end{array}$ & $\begin{array}{r}37^{\circ} 31.52^{\prime} \\
-122^{\circ} 8.51^{\prime}\end{array}$ & 5.2 & $\begin{array}{l}3.0 \mathrm{~m} \text { barrel } \\
(\mathrm{G})\end{array}$ & 2.15 & $\begin{array}{l}\text { free fall, buried the head, } \\
\text { bottom: fairly clean mud, top: } \\
\text { real clean mud, a few burrows }\end{array}$ \\
\hline 23 & $4-\mathrm{G}-25$ & $\begin{array}{l}\text { Up slope of } \# 22 \text { on the } \\
\text { subtidal flat }\end{array}$ & $\begin{array}{r}37^{\circ} 31.59^{\prime} \\
-122^{\circ} 8.44^{\prime}\end{array}$ & 7.2 & $\begin{array}{l}2.5 \mathrm{~m} \text { barrel } \\
(\mathrm{G})\end{array}$ & 1.76 & $\begin{array}{l}\text { buried head, clean mud at the } \\
\text { bottom, top: clean mud, a few } \\
\text { burrows }\end{array}$ \\
\hline
\end{tabular}




\begin{tabular}{|c|c|c|c|c|c|c|c|}
\hline $\begin{array}{l}\text { Мap } \\
\#\end{array}$ & $\begin{array}{l}\text { Core } \\
\text { Number }\end{array}$ & Setting & $\begin{array}{c}\text { Lat. } \\
\text { Long. }\end{array}$ & $\begin{array}{l}\text { Water } \\
\text { Depth (m) }\end{array}$ & $\begin{array}{l}\text { Type } \\
\text { Core (G) } \\
\text { (B) }\end{array}$ & $\begin{array}{l}\text { Core } \\
\text { length } \\
\text { (m) }\end{array}$ & General Description \\
\hline
\end{tabular}

\begin{tabular}{|c|c|c|c|c|c|c|c|}
\hline 24 & $5-\mathrm{G}-25$ & $\begin{array}{l}\text { Along the break in slope just } \\
\text { above the thalweg south of the } \\
\text { mouth of Redwood Creek }\end{array}$ & $\begin{array}{r}37^{\circ} 31.82^{\prime} \\
-122^{\circ} 9.74^{\prime}\end{array}$ & 6.2 & $\begin{array}{l}3.0 \mathrm{~m} \text { barrel } \\
(\mathrm{G})\end{array}$ & 1.51 & $\begin{array}{l}\text { let down slowly, shells at the } \\
\text { bottom, shells at the top }\end{array}$ \\
\hline 25 & $6-\mathrm{G}-25$ & $\begin{array}{l}\text { South of the mouth of } \\
\text { Redwood Creek in line with } \\
\# 24 \text { in } 4.7 \text { m of water, south of } \\
\# 26\end{array}$ & $\begin{array}{r}37^{\circ} 31.72^{\prime} \\
-122^{\circ} 9.90^{\prime}\end{array}$ & 4.7 & $\begin{array}{l}2.5 \mathrm{~m} \text { barrel } \\
(\mathrm{G})\end{array}$ & .75 & $\begin{array}{l}\text { two attempts, shell on bottom: } \\
\text { slightly sandy silt, top: shells }\end{array}$ \\
\hline 26 & $7-\mathrm{G}-25$ & $\begin{array}{l}\text { South of the mouth of } \\
\text { Redwood Creek north of } \# 25 \\
\text { along the channel slope }\end{array}$ & $\begin{array}{r}37^{\circ} 31.83^{\prime} \\
-122^{\circ} 10.07^{\prime}\end{array}$ & 6 & $\begin{array}{l}2.5 \mathrm{~m} \text { barrel } \\
(\mathrm{G})\end{array}$ & 2.01 & $\begin{array}{l}\text { free fall, sandy silt, top: worm } \\
\text { tubes, shells }\end{array}$ \\
\hline 27 & $8-\mathrm{G}-25$ & $\begin{array}{l}\text { South of the mouth of } \\
\text { Redwood Creek north of \#26 } \\
\text { along the channel slope in } \\
\text { deeper water }\end{array}$ & $\begin{array}{r}37^{\circ} 31.96^{\prime} \\
-122^{\circ} 10.21^{\prime}\end{array}$ & 7.5 & $\begin{array}{l}2.5 \mathrm{~m} \text { barrel } \\
(\mathrm{G})\end{array}$ & 2.11 & $\begin{array}{l}\text { free fall, bottom: firm mud, } \\
\text { top: small shell fragments } \\
\text { worm tubes, sand }\end{array}$ \\
\hline 28 & $9-\mathrm{G}-25$ & $\begin{array}{l}\text { Adjacent to the mouth of } \\
\text { Redwood Creek south of the } \\
\text { shoals. }\end{array}$ & $\begin{array}{r}37^{\circ} 32.47^{\prime} \\
-122^{\circ} 11.18^{\prime}\end{array}$ & 3.25 & $\begin{array}{l}3.0 \mathrm{~m} \text { barrel } \\
(\mathrm{G})\end{array}$ & 1.08 & bottom - shells top: shells \\
\hline 29 & $10-\mathrm{G}-25$ & $\begin{array}{l}\text { Down slope of \#28 near the } \\
\text { Redwood Creek mouth shoals }\end{array}$ & $\begin{array}{r}37^{\circ} 32.67^{\prime} \\
-122^{\circ} 11.15^{\prime} \\
\end{array}$ & 6.1 & $\begin{array}{l}3.0 \mathrm{~m} \text { barrel } \\
(\mathrm{G})\end{array}$ & 1.80 & $\begin{array}{l}\text { bottom: stiff mud, no shells, } \\
\text { top: firm mud, shells }\end{array}$ \\
\hline 30 & $11-\mathrm{G}-25$ & $\begin{array}{l}\text { On top of the Redwood Creek } \\
\text { mouth shoals south of the } \\
\text { mouth }\end{array}$ & $\begin{array}{r}37^{\circ} 32.66^{\prime} \\
-122^{\circ} 11.41^{\prime}\end{array}$ & 3.9 & $\begin{array}{l}3.0 \mathrm{~m} \text { barrel } \\
(\mathrm{G})\end{array}$ & 2.85 & $\begin{array}{l}\text { buried head, bottom: firm mud, } \\
\text { top: mud, shells }\end{array}$ \\
\hline 31 & $12-\mathrm{G}-25$ & $\begin{array}{l}\text { Down slope of \#30 south of } \\
\text { the mouth of Redwood Ck. }\end{array}$ & $\begin{array}{rr}37^{\circ} & 32.77^{\prime} \\
-122^{\circ} & 11.40^{\prime} \\
\end{array}$ & 11.2 & $\begin{array}{l}3.0 \mathrm{~m} \text { barrel } \\
(\mathrm{G})\end{array}$ & 1.63 & $\begin{array}{l}\text { bottom: firm mud, top: firm } \\
\text { mud, shells }\end{array}$ \\
\hline 32 & $13-\mathrm{G}-25$ & $\begin{array}{l}\text { South of the mouth of } \\
\text { Redwood Ck.,south of \#28 } \\
\text { near the thalweg }\end{array}$ & $\begin{array}{r}37^{\circ} 32.47^{\prime} \\
-122^{\circ} 10.85^{\prime}\end{array}$ & 12.5 & $\begin{array}{l}3.0 \mathrm{~m} \text { barrel } \\
(\mathrm{G})\end{array}$ & .40 & $\begin{array}{l}\text { problems: short take, bottom: } \\
\text { clay with shell material, top: } \\
\text { worms, shell, not sure core } \\
\text { went in vertically }\end{array}$ \\
\hline 33 & $14-\mathrm{G}-25$ & $\begin{array}{l}\text { Down slope of } \# 31 \text { adjacent to } \\
\text { the mouth of Redwood Ck. }\end{array}$ & $\begin{array}{r}37^{\circ} 32.95^{\prime} \\
-122^{\circ} 11.09^{\prime} \\
\end{array}$ & 4.2 & $\begin{array}{l}3.0 \mathrm{~m} \text { barrel } \\
(\mathrm{G})\end{array}$ & .62 & $\begin{array}{l}\text { let down slowly, bottom: shell } \\
\text { hash top: shell, }\end{array}$ \\
\hline 34 & $1-\mathrm{G}-26$ & $\begin{array}{l}\text { Along Northeast side of the } \\
\text { main channel on the slope of } \\
\text { the channel in line with } \\
\# 30,31, \& 33\end{array}$ & $\begin{array}{r}37^{\circ} 33.31^{\prime} \\
-122^{\circ} 10.70^{\prime}\end{array}$ & 9.2 & $\begin{array}{l}2.5 \mathrm{~m} \text { barrel } \\
(\mathrm{G})\end{array}$ & 2.14 & $\begin{array}{l}\text { bottom: cohesive stiff clay } \\
\text { mud top: shell hash, worm } \\
\text { tubes }\end{array}$ \\
\hline 35 & $2-\mathrm{G}-26$ & $\begin{array}{l}\text { Near the thalweg of the main } \\
\text { channel in-line with } \# 28, \& 29 .\end{array}$ & $\begin{array}{r}37^{\circ} 32.79^{\prime} \\
-122^{\circ} 11.07^{\prime}\end{array}$ & 10.5 & $\begin{array}{l}2.5 \mathrm{~m} \text { barrel } \\
(\mathrm{G})\end{array}$ & $2.20+.25$ & $\begin{array}{l}\text { lost about } 20 \mathrm{~cm} \text { of bottom of } \\
\text { the core: cohesive mud, top: } \\
\text { some shelly material }\end{array}$ \\
\hline
\end{tabular}




\begin{tabular}{|c|c|c|c|c|c|c|c|}
\hline Мap & $\begin{array}{l}\text { Core } \\
\text { Number }\end{array}$ & Setting & $\begin{array}{l}\text { Lat. } \\
\text { Long. }\end{array}$ & $\begin{array}{l}\text { Water } \\
\text { Depth (m) }\end{array}$ & $\begin{array}{l}\text { Type } \\
\text { Core (G) } \\
\text { (B) } \\
\end{array}$ & \begin{tabular}{|l|} 
Core \\
length \\
$(\mathrm{m})$ \\
\end{tabular} & General Description \\
\hline 36 & $3-\mathrm{G}-26$ & $\begin{array}{l}\text { Near the Thalweg along } \\
\text { northeast side of the channel } \\
\text { in-line with \#35 }\end{array}$ & $\begin{array}{r}37^{\circ} 33.01^{\prime} \\
-122^{\circ} 10.82^{\prime}\end{array}$ & 14 & $\begin{array}{l}3.0 \mathrm{~m} \text { barrel } \\
(\mathrm{G})\end{array}$ & 2.03 & $\begin{array}{l}\text { free fall, bottom: sand fairly } \\
\text { well sorted }\end{array}$ \\
\hline 37 & 4-G-26 & Upslope and north of \#36 & $\begin{array}{r}37^{\circ} 33.21^{\prime} \\
-122^{\circ} 10.93^{\prime}\end{array}$ & 10.5 & $\begin{array}{l}3.0 \mathrm{~m} \text { barrel } \\
(\mathrm{G})\end{array}$ & 1.88 & $\begin{array}{l}\text { free fall, bottom: shelly mud, } \\
\text { top: silt? with some shell } \\
\text { fragments }\end{array}$ \\
\hline 38 & $5-\mathrm{G}-26$ & $\begin{array}{l}\text { Near the top of the Northeast } \\
\text { channel margin upslope of } \# 34\end{array}$ & $\begin{array}{r}37^{\circ} 33.51^{\prime} \\
-122^{\circ} 10.64^{\prime}\end{array}$ & 5.5 & $\begin{array}{l}3.0 \text { m barrel } \\
(\mathrm{G})\end{array}$ & 2.20 & $\begin{array}{l}\text { free fall, bottom: mud, some } \\
\text { shells }\end{array}$ \\
\hline
\end{tabular}

\begin{tabular}{|c|c|c|c|c|c|c|c|}
\hline \multicolumn{8}{|c|}{$\begin{array}{c}\text { J-2-90-SF } \\
\text { San Joaquin River } \\
\text { Map P }\end{array}$} \\
\hline 39 & SFB013090-1 & $\begin{array}{l}\text { San Joaquin River from } \\
\text { Marker \#39 shallow water on } \\
\text { bank of point bar }\end{array}$ & $\begin{array}{r}38^{\circ} 6.06^{\prime} \\
-121^{\circ} 36.70^{\prime}\end{array}$ & $4.0-4.5$ & $\begin{array}{l}2.5 \mathrm{~m} \text { barrel } \\
(\mathrm{G})\end{array}$ & 1.50 & silty sand \\
\hline 40 & SFB013090-2 & $\begin{array}{l}\text { South west of core \#39 in } 6.5 \\
m \text { water no core penetration }\end{array}$ & $\begin{array}{r}38^{\circ} 5.43^{\prime} \\
-121^{\circ} 37.40^{\prime}\end{array}$ & & $\begin{array}{l}2.5 \mathrm{~m} \text { barrel } \\
(\mathrm{G})\end{array}$ & & $\begin{array}{l}\text { sample consist of bag sample } \\
\text { no penetration with both box } \\
\text { and gravity core fine silty sand } \\
\text { on bottom. }\end{array}$ \\
\hline 41 & SFB013090-3 & Station south of Marker \#38 & $\begin{array}{r}38^{\circ} 5.61^{\prime} \\
-121^{\circ} 37.49^{\prime} \\
\end{array}$ & 6.0 & $\begin{array}{l}2.5 \mathrm{~m} \text { barrel } \\
(\mathrm{G})\end{array}$ & 1.50 & $\begin{array}{l}\text { sandy in top } 30 \mathrm{~cm} \text {, muddy } \\
\text { below }\end{array}$ \\
\hline 42 & SFB013090-4 & On channel bank & $\begin{array}{r}38^{\circ} 5.55^{\prime} \\
-121^{\circ} 38.90^{\prime} \\
\end{array}$ & 4.2 & $\begin{array}{l}2.5 \mathrm{~m} \text { barrel } \\
(\mathrm{G})\end{array}$ & & \\
\hline 43 & SFB013090-5 & Point bar upstream end & $\begin{array}{r}38^{\circ} 5.73^{\prime} \\
-121^{\circ} 39.80^{\prime} \\
\end{array}$ & 4.0 & $\begin{array}{l}2.5 \mathrm{~m} \text { barrel } \\
(\mathrm{G})\end{array}$ & & at edge of tules \\
\hline
\end{tabular}

\section{J-2-90-SF}

Sacramento River from Northeast of Rio Vista to the San Joaquin River

Map O, P \& Q

\begin{tabular}{|c|c|c|c|c|c|c|c|}
\hline \multicolumn{8}{|c|}{ Мар O, P \& Q } \\
\hline 44 & SFB013190-1 & $\begin{array}{l}\text { Sacramento River from up- } \\
\text { stream of Marker \#41 near } \\
\text { dredged channel }\end{array}$ & $\begin{array}{r}38^{\circ} 11.49^{\prime} \\
-121^{\circ} 39.36^{\prime}\end{array}$ & 13.5 & $\begin{array}{l}1.0 \mathrm{~m} \text { barrel } \\
\text { (G) }\end{array}$ & $\sim .25$ & stiff mud overlain by mud \\
\hline 45 & SFB013190-2 & $\begin{array}{l}\text { Up Steam Boat slough, in sand } \\
\text { waves }\end{array}$ & $\begin{array}{r}38^{\circ} 10.97^{\prime} \\
-121^{\circ} 39.25^{\prime}\end{array}$ & 1.8 & $\begin{array}{l}1.0 \mathrm{~m} \text { barrel } \\
\text { (G) }\end{array}$ & $\begin{array}{l}\text { bagged } \\
\text { sample }\end{array}$ & coarse sand \\
\hline
\end{tabular}




\begin{tabular}{|c|c|c|c|c|c|c|c|}
\hline $\begin{array}{l}\text { Мap } \\
\#\end{array}$ & $\begin{array}{l}\text { Core } \\
\text { Number }\end{array}$ & Setting & $\begin{array}{c}\text { Lat. } \\
\text { Long. }\end{array}$ & $\begin{array}{l}\text { Water } \\
\text { Depth (m) }\end{array}$ & $\begin{array}{l}\text { Type } \\
\text { Core (G) } \\
\text { (B) }\end{array}$ & $\begin{array}{l}\text { Core } \\
\text { length } \\
\text { (m) }\end{array}$ & General Description \\
\hline
\end{tabular}

\begin{tabular}{|c|c|c|c|c|c|c|c|}
\hline 46 & SFB013190-3 & $\begin{array}{l}\text { Upper part of small bar, very } \\
\text { steep bank }\end{array}$ & $\begin{array}{r}38^{\circ} 10.60^{\prime} \\
-121^{\circ} 39.88^{\prime}\end{array}$ & 1.5 & $\begin{array}{l}1.0 \mathrm{~m} \text { barrel } \\
(\mathrm{G})\end{array}$ & $\begin{array}{l}\text { very little } \\
\text { penetratio } \\
\mathrm{n}\end{array}$ & core much disturbed \\
\hline 47 & SFB013190-4 & From N. bank & $\begin{array}{r}38^{\circ} 10.09^{\prime} \\
-121^{\circ} 40.25^{\prime}\end{array}$ & 2.8 & $\begin{array}{l}1.0 \mathrm{~m} \text { barrel } \\
(\mathrm{G})\end{array}$ & .50 & good core \\
\hline 48 & SFB013190-5 & $\begin{array}{l}\text { On the upper part of a bank } \\
\text { protected from upstream flow }\end{array}$ & $\begin{array}{r}38^{\circ} 9.61^{\prime} \\
-121^{\circ} 40.55^{\prime}\end{array}$ & 2.3 & $\begin{array}{l}1.0 \mathrm{~m} \text { barrel } \\
(\mathrm{G})\end{array}$ & .30 & $\begin{array}{l}\text { badly disturbed sample fairly } \\
\text { coarse sand }\end{array}$ \\
\hline 49 & SFB013190-6 & $\begin{array}{l}\text { Decker Island on the S. side of } \\
\text { the island }\end{array}$ & $\begin{array}{r}38^{\circ} 4.99^{\prime} \\
-121^{\circ} 43.56^{\prime}\end{array}$ & 2.5 & $\begin{array}{l}1.5 \mathrm{~m} \text { barrel } \\
\text { (G) }\end{array}$ & 1.50 & fine densely packed sand \\
\hline 50 & SFB013190-7 & Same site as \#49 & $\begin{array}{r}38^{\circ} 4.98^{\prime} \\
-121^{\circ} 43.48^{\prime} \\
\end{array}$ & 2.5 & $1.5 \mathrm{~m}$ barrel $(\mathrm{G})$ & 2.50 & soupy top \\
\hline 51 & SFB013190-8 & Same site as \#50 & $\begin{array}{r}38^{\circ} 4.93^{\prime} \\
-121^{\circ} 43.66^{\prime} \\
\end{array}$ & 4.5 & $\begin{array}{l}2.5 \mathrm{~m} \text { barrel } \\
(\mathrm{G})\end{array}$ & & \\
\hline 52 & SFB013190-9 & $\begin{array}{l}\text { Base of slope below \#50, and } \\
51\end{array}$ & $\begin{array}{r}38^{\circ} 4.87^{\prime} \\
-121^{\circ} 43.48^{\prime}\end{array}$ & 7.2 & $\begin{array}{l}2.5 \mathrm{~m} \text { barrel } \\
(\mathrm{G})\end{array}$ & 1.5 & $\begin{array}{l}\text { filled core barrel, losing the } \\
\text { top } 15-20 \mathrm{~cm}\end{array}$ \\
\hline 53 & SFB013190-10 & $\begin{array}{l}\text { In thalweg vicinity of } \# 51,52, \\
\& 53\end{array}$ & $\begin{array}{r}38^{\circ} 4.84^{\prime} \\
-121^{\circ} 43.61^{\prime}\end{array}$ & 8.5 & $\begin{array}{l}2.5 \mathrm{~m} \text { barrel } \\
(\mathrm{G})\end{array}$ & .80 & $\begin{array}{l}\text { disturbed coarse sand at the } \\
\text { top, mud then coarse sand at } \\
\text { the base }\end{array}$ \\
\hline 54 & SFB013190-11 & $\begin{array}{l}\text { Flat bottom, no sand waves } \\
\text { near N. bank of Sacramento. } \\
\text { R. }\end{array}$ & $\begin{array}{r}38^{\circ} 5.23^{\prime} \\
-121^{\circ} 44.81^{\prime}\end{array}$ & 7.3 & $\begin{array}{l}1.5 \mathrm{~m} \text { barrel } \\
(\mathrm{G})\end{array}$ & 1.5 & buried head found mud \\
\hline 55 & SFB013190-12 & Same location as $\# 54$ & $\begin{array}{r}38^{\circ} 5.05^{\prime} \\
-121^{\circ} 45.20^{\prime}\end{array}$ & 7.3 & $\begin{array}{l}2.5 \mathrm{~m} \text { barrel } \\
(\mathrm{G})\end{array}$ & 2.5 & $\begin{array}{l}\text { peat (wood detritus) in bottom } \\
\text { of both cores loss in the top of } \\
\text { the short barrel. }\end{array}$ \\
\hline
\end{tabular}

\section{J-2-90-SF}

Suisun Bay in the vicinity of Roe and Ryer Island

Map M

\begin{tabular}{|c|c|c|c|c|c|c|c|}
\hline 56 & SFB020190-1 & $\begin{array}{l}\text { SE of Dutton Island in Honker } \\
\text { Bay }\end{array}$ & $\begin{array}{r}38^{\circ} 4.10^{\prime} \\
-121^{\circ} 57.72^{\prime}\end{array}$ & 3 & $\begin{array}{l}3.0 \mathrm{~m} \text { barrel } \\
(\mathrm{G})\end{array}$ & 1.55 & free fall, flat smooth bottom \\
\hline 57 & SFB020190-2 & Further up Honker Bay & $\begin{array}{r}38^{\circ} 4.35^{\prime} \\
-121^{\circ} 57.38^{\prime}\end{array}$ & 2.7 & $\begin{array}{l}3.0 \mathrm{~m} \text { barrel } \\
(\mathrm{G})\end{array}$ & 1.63 & $\begin{array}{l}\text { flat surface above smooth } \\
\text { sloping muddy bank }\end{array}$ \\
\hline 58 & SFB020190-3 & $\begin{array}{l}\text { Margin of Honker Bay } \\
\text { between sites } \# 56 \& 58\end{array}$ & $\begin{array}{r}38^{\circ} 3.73^{\prime} \\
-121^{\circ} 57.43^{\prime}\end{array}$ & 2.8 & $\begin{array}{l}3.0 \mathrm{~m} \text { barrel } \\
(\mathrm{G})\end{array}$ & 1.94 & between sites $1 \& 3$ \\
\hline
\end{tabular}




\begin{tabular}{|c|c|c|c|c|c|c|c|}
\hline $\begin{array}{l}\text { Map } \\
\#\end{array}$ & $\begin{array}{l}\text { Core } \\
\text { Number }\end{array}$ & Setting & $\begin{array}{l}\text { Lat. } \\
\text { Long. }\end{array}$ & $\begin{array}{l}\text { Water } \\
\text { Depth (m) }\end{array}$ & $\begin{array}{l}\text { Type } \\
\text { Core (G) } \\
\text { (B) } \\
\end{array}$ & \begin{tabular}{|l|} 
Core \\
length \\
$(\mathrm{m})$ \\
\end{tabular} & General Description \\
\hline 59 & SFB020190-4 & $\begin{array}{l}\text { Channel floor E. of Snag } \\
\text { Island. Sand waves on } \\
\text { fathometer }\end{array}$ & $\begin{array}{r}38^{\circ} 4.57^{\prime} \\
-121^{\circ} 59.42^{\prime}\end{array}$ & 5.5 & $\begin{array}{l}1.75 \mathrm{~m} \text { barrel } \\
(\mathrm{G})\end{array}$ & .60 & $\begin{array}{l}\text { smooth floor below platform } \\
\text { w/sand waves }\end{array}$ \\
\hline 60 & SFB020190-5 & $\begin{array}{l}\text { Channel of Pt. Buckler feeding } \\
\text { into Grizzly Bay. }\end{array}$ & $\begin{array}{r}38^{\circ} 5.67^{\prime} \\
-122^{\circ} 1.16^{\prime} \\
\end{array}$ & 9 & $\begin{array}{l}2.5 \mathrm{~m} \text { barrel, } \\
(\mathrm{G})\end{array}$ & 1.30 & sand at top \\
\hline 61 & SFB020190-6 & $\begin{array}{l}\text { On sloping bank midway in } \\
\text { Grizzly Bay mouth }\end{array}$ & $\begin{array}{r}38^{\circ} 6.06^{\prime} \\
-122^{\circ} 1.58^{\prime} \\
\end{array}$ & 3.6 & $\begin{array}{l}2.5 \mathrm{~m} \text { barrel, } \\
(\mathrm{G})\end{array}$ & 2.05 & $\begin{array}{l}\text { wood detritus in bottom, } \\
\text { bagged sample }\end{array}$ \\
\hline 62 & SFB020190-7 & $\begin{array}{l}\text { Between mouth of Suisun } \\
\text { Slough \& Garnet Pt. on Ryer } \\
\text { Island, on high in channel } \\
\text { center }\end{array}$ & $\begin{array}{r}38^{\circ} 6.27^{\prime} \\
-122^{\circ} 2.93^{\prime}\end{array}$ & 3 & $\begin{array}{l}2.5 \mathrm{~m} \text { barrel, } \\
(\mathrm{G})\end{array}$ & 2.01 & $18 \mathrm{~cm}$ sand at top \\
\hline 63 & SFB020190-8 & Inshore in Grizzly Bay & $\begin{array}{r}38^{\circ} 6.47^{\prime} \\
-122^{\circ} 2.97^{\prime}\end{array}$ & 2 & $\begin{array}{l}3.0 \mathrm{~m} \text { barrel } \\
\text { (G) }\end{array}$ & 3.05 & $\begin{array}{l}\text { filled the } 3.0 \mathrm{~m} \text { barrel }(\mathrm{G}), \\
\text { mud } 10-20 \mathrm{~cm} \text { ? loss of the } \\
\text { top of core }\end{array}$ \\
\hline 64 & SFB020190-9 & $\begin{array}{l}\text { On ridge extending } \mathrm{SW} \text { into } \\
\text { main Suisun Bay channel } \\
\text { (shoal extension of Garnet Pt. }\end{array}$ & $\begin{array}{r}38^{\circ} 5.39^{\prime} \\
-122^{\circ} 3.83^{\prime}\end{array}$ & 2.3 & $\begin{array}{l}1.75 \mathrm{~m} \text { barrel } \\
\text { (G) }\end{array}$ & .76 & \\
\hline 65 & SFB020190-10 & $\begin{array}{l}\text { At the tip of shoal in the } \\
\text { middle of Susiun Bay south of } \\
\text { the Mothball fleet }\end{array}$ & $\begin{array}{r}38^{\circ} 4.35^{\prime} \\
-122^{\circ} 5.16^{\prime}\end{array}$ & 3.3 & $\begin{array}{l}2.5 \mathrm{~m} \text { barrel, } \\
(\mathrm{G})\end{array}$ & 1.69 & $\begin{array}{l}\text { sand in upper part of core } \\
\text { (liquidized) organic detritus in } \\
\text { base }\end{array}$ \\
\hline 66 & SFB020190-11 & $\begin{array}{l}\text { Old geochemical site., Sand } \\
\text { waves seen on fathometer }\end{array}$ & $\begin{array}{r}38^{\circ} 3.45^{\prime} \\
-122^{\circ} 5.91^{\prime}\end{array}$ & 3.5 & $\begin{array}{l}2.5 \mathrm{~m} \text { barrel, } \\
(\mathrm{G})\end{array}$ & $\mathrm{N} / \mathrm{C}$ & $\begin{array}{l}\text { bag sample much washing not } \\
\text { enough stratigraphic coherence } \\
\text { to keep }\end{array}$ \\
\hline 67 & SFB020190-12 & $\begin{array}{l}\text { Southeast of \#65 on sand } \\
\text { shoals }\end{array}$ & $\begin{array}{r}38^{\circ} 4.16^{\prime} \\
-122^{\circ} 4.54^{\prime} \\
\end{array}$ & 3.2 & $\begin{array}{l}2.5 \mathrm{~m} \text { barrel, } \\
(\mathrm{G})\end{array}$ & 2.30 & \\
\hline 68 & SFB020190-13 & $\begin{array}{l}\text { Just North over divide into } \\
\text { channel between Roe \& Ryer } \\
\text { Islands }\end{array}$ & $\begin{array}{r}38^{\circ} 4.99^{\prime} \\
-122^{\circ} 3.20^{\prime}\end{array}$ & 4.5 & & & \\
\hline 69 & SFB020190-14 & N. of Roe Island on bank & $\begin{array}{r}38^{\circ} 4.64^{\prime} \\
-122^{\circ} 1.85^{\prime} \\
\end{array}$ & 4.0 & $\begin{array}{l}2.5 \mathrm{~m} \text { barrel, } \\
(\mathrm{G})\end{array}$ & .35 & $\begin{array}{l}\text { poor penetration, sand waves } \\
\text { on fathometer }\end{array}$ \\
\hline 70 & SFB020190-15 & $\begin{array}{l}\text { S. side Ryer Island base of } \\
\text { channel }\end{array}$ & $\begin{array}{r}38^{\circ} 4.43^{\prime} \\
-122^{\circ} 1.07^{\prime}\end{array}$ & 5.5 & $\begin{array}{l}1.75 \mathrm{~m} \text { barrel } \\
\text { (G) }\end{array}$ & 1.07 & $\begin{array}{l}\text { sand with some wood at the } \\
\text { base }\end{array}$ \\
\hline
\end{tabular}




\begin{tabular}{|c|c|c|c|c|c|c|c|}
\hline $\begin{array}{l}\text { Map } \\
\#\end{array}$ & $\begin{array}{l}\text { Core } \\
\text { Number }\end{array}$ & Setting & $\begin{array}{l}\text { Lat. } \\
\text { Long. }\end{array}$ & $\begin{array}{l}\text { Water } \\
\text { Depth (m) }\end{array}$ & $\begin{array}{l}\text { Type } \\
\text { Core (G) } \\
\text { (B) }\end{array}$ & $\begin{array}{l}\text { Core } \\
\text { length } \\
(\mathrm{m})\end{array}$ & General Description \\
\hline
\end{tabular}

\begin{tabular}{|c|c|c|c|c|c|c|c|}
\hline \multicolumn{8}{|c|}{$\begin{array}{c}\text { J-2-90-SF } \\
\text { San Joaquin River near the confluence with the Sacramento River } \\
\text { area of Fraser Shoal } \\
\text { Map N }\end{array}$} \\
\hline 71 & SFB020290-1 & $\begin{array}{l}\text { W.bank (steep bank of broad } \\
\text { slough., Nose of boat almost } \\
\text { on bank }\end{array}$ & $\begin{array}{r}38^{\circ} 3.25^{\prime} \\
-121^{\circ} 50.76^{\prime}\end{array}$ & 1.1 & $\begin{array}{l}2.5 \mathrm{~m} \text { barrel, } \\
(\mathrm{G})\end{array}$ & 1.52 & mostly organic hash \\
\hline 72 & SFB020290-2 & $\begin{array}{l}\text { On W. side of Medial shoal in } \\
\text { Broad Slough } \sim 1 / 2 \text { up slough } \\
\text { smooth bottom gradual incline }\end{array}$ & $\begin{array}{r}38^{\circ} 2.84^{\prime} \\
-121^{\circ} 50.33^{\prime}\end{array}$ & 2.75 & $\begin{array}{l}1.75 \mathrm{~m} \text { barrel } \\
(\mathrm{G})\end{array}$ & 1.70 & $\begin{array}{l}\text { head partly buried in mud, } \\
\text { sandy at top, sandy silt at base. }\end{array}$ \\
\hline 73 & SFB020290-3 & $\begin{array}{l}\text { W. bank of Broad Slough on } \\
\text { steep slope }\end{array}$ & $\begin{array}{r}38^{\circ} 2.27^{\prime} \\
-121^{\circ} 50.48^{\prime} \\
\end{array}$ & 3.5 & $\begin{array}{l}2.5 \mathrm{~m} \text { barrel, } \\
(\mathrm{G})\end{array}$ & 1.04 & organic rich top and bottom \\
\hline 74 & SFB020290-4 & NW bank of Kimball Island & $\begin{array}{r}38^{\circ} 1.97^{\prime} \\
-121^{\circ} 49.54^{\prime} \\
\end{array}$ & 2.5 & $\begin{array}{l}2.5 \mathrm{~m} \text { barrel, } \\
(\mathrm{G})\end{array}$ & .60 & sand and mud \\
\hline 75 & SFB020290-5 & $\begin{array}{l}\text { E channel of Broad Slough, S. } \\
\text { end sand waves }\end{array}$ & $\begin{array}{r}38^{\circ} 2.04^{\prime} \\
-121^{\circ} 49.78^{\prime} \\
\end{array}$ & 4.7 & $\begin{array}{l}2.5 \mathrm{~m} \text { barrel, } \\
(\mathrm{G})\end{array}$ & .20 & sand \\
\hline 76 & SFB02029--6 & $\begin{array}{l}\text { Channel floor E. channel of } \\
\text { Broad Slough }\end{array}$ & $\begin{array}{r}38^{\circ} 2.20^{\prime} \\
-121^{\circ} 49.94^{\prime} \\
\end{array}$ & 7.4 & $\begin{array}{l}2.5 \mathrm{~m} \text { barrel, } \\
(\mathrm{G})\end{array}$ & 1.03 & $7 \mathrm{~cm}$ sand at top sharply/mud \\
\hline 77 & SFB020290-7 & $\begin{array}{l}\text { E. bank of channel at location } \\
\text { of \# } 77 \text { upper bank }\end{array}$ & $\begin{array}{r}38^{\circ} 2.30^{\prime} \\
-121^{\circ} 49.75^{\prime} \\
\end{array}$ & 2.2 & $\begin{array}{l}2.5 \mathrm{~m} \text { barrel, } \\
(\mathrm{G})\end{array}$ & 1.90 & base of head buried/mud \\
\hline 78 & SFB020290-8 & $\begin{array}{l}\text { W.bank of channel (Fraser } \\
\text { shoal side) }\end{array}$ & $\begin{array}{r}38^{\circ} 2.48^{\prime} \\
-121^{\circ} 50.19^{\prime} \\
\end{array}$ & 3.2 & $\begin{array}{l}2.5 \mathrm{~m} \text { barrel, } \\
(\mathrm{G})\end{array}$ & 1.98 & organics at base good core \\
\hline 79 & SFB020290-9 & $\begin{array}{l}\text { W bank of channel behind } \\
\text { Chain Island }\end{array}$ & $\begin{array}{r}38^{\circ} 4.18^{\prime} \\
-121^{\circ} 51.49^{\prime}\end{array}$ & & $\begin{array}{l}2.5 \mathrm{~m} \text { barrel, } \\
\text { (G) }\end{array}$ & 2.26 & $\mathrm{mud} /$ penetration \\
\hline 80 & SFB020290-10 & $\begin{array}{l}\text { NW side of Browns Island- lee } \\
\text { on depositional slope irregular } \\
\text { bathymetry slumps? sand } \\
\text { waves? (slumps? on side scan } \\
\text { record) }\end{array}$ & $\begin{array}{r}38^{\circ} 2.72^{\prime} \\
-121^{\circ} 52.39^{\prime}\end{array}$ & 3.4 & $\begin{array}{l}2.5 \mathrm{~m} \text { barrel, } \\
(\mathrm{G})\end{array}$ & $\mathrm{N} / \mathrm{C}$ & $\begin{array}{l}\text { No penetration no core sandy } \\
\text { on core bottom bagged sand }\end{array}$ \\
\hline
\end{tabular}

\section{J-2-90-SF}

Suisun Bay, South of Ryer Island to Seal Island

Map M

$81 \quad$ SFB020590-1 On S. side of shoal extending

SE from Ryer Island in $\sim 3 \mathrm{~m}$

$\begin{array}{rll}38^{\circ} 4.48^{\prime} & 2.75 & 2.5 \mathrm{~m} \text { barrel, } \\ -121^{\circ} 59.58^{\prime} & & (\mathrm{G})\end{array}$

2.15

good penetration head partly

buried in mud 


\begin{tabular}{|c|c|c|c|c|c|c|c|}
\hline $\begin{array}{l}\text { Мap } \\
\#\end{array}$ & $\begin{array}{l}\text { Core } \\
\text { Number }\end{array}$ & Setting & $\begin{array}{c}\text { Lat. } \\
\text { Long. }\end{array}$ & $\begin{array}{l}\text { Water } \\
\text { Depth (m) }\end{array}$ & $\begin{array}{l}\text { Type } \\
\text { Core (G) } \\
\text { (B) }\end{array}$ & $\begin{array}{l}\text { Core } \\
\text { length } \\
\text { (m) }\end{array}$ & General Description \\
\hline
\end{tabular}

\begin{tabular}{|c|c|c|c|c|c|c|c|}
\hline 82 & SFB020590-2 & Channel floor & $\begin{array}{r}38^{\circ} 4.37^{\prime} \\
-121^{\circ} 59.63^{\prime} \\
\end{array}$ & 6.6 & $\begin{array}{l}1.25 \mathrm{~m} \text { barrel } \\
(\mathrm{G})\end{array}$ & .22 & sand/sand mud \\
\hline 83 & SFB020590-3 & $\begin{array}{l}\text { On N. bank of Middle Ground } \\
\mathrm{S} \text { of SE end of Ryer Island v. } \\
\text { irregular bottom-excavation? }\end{array}$ & $\begin{array}{r}38^{\circ} 4.06^{\prime} \\
-121^{\circ} 59.28^{\prime}\end{array}$ & 3 & $\begin{array}{l}2.5 \mathrm{~m} \text { barrel, } \\
(\mathrm{G})\end{array}$ & 1.13 & $\begin{array}{l}38 \mathrm{~cm} \text { sand, sand waves ebb } \\
\text { oriented } 5 \mathrm{~m} \text { depth channel off } \\
\text { NE end Middle Ground }\end{array}$ \\
\hline 84 & SFB020590-4 & NE end of Middle Ground & $\begin{array}{r}38^{\circ} 3.99^{\prime} \\
-121^{\circ} 58.88^{\prime} \\
\end{array}$ & 3.5 & $\begin{array}{l}1.75 \mathrm{~m} \text { barrel } \\
(\mathrm{G})\end{array}$ & $\sim .30$ & sand/mud \\
\hline 85 & SFB020590-5 & $\begin{array}{l}\text { S bank Middle Shoal between } \\
\# 17 \text { \& \# } 19\end{array}$ & $\begin{array}{r}38^{\circ} 4.12^{\prime} \\
-122^{\circ} 0.16^{\prime}\end{array}$ & 2.2 & $\begin{array}{l}1.75 \mathrm{~m} \text { barrel } \\
\text { (G) }\end{array}$ & $\mathrm{N} / \mathrm{C}$ & $\begin{array}{l}\text { no penetration shells separated } \\
\text { valves disarticulated in both } 4^{\prime} \\
\& 2.5 \mathrm{~m} \text { barrel }(\mathrm{G}) \text { cast (bag } \\
\text { sample) }\end{array}$ \\
\hline 86 & SFB020590-6 & $\begin{array}{l}\text { Shoal area W. of Seal Island } \\
\text { smooth bottom sloping bank }\end{array}$ & $\begin{array}{r}38^{\circ} 3.50^{\prime} \\
-122^{\circ} 2.87^{\prime}\end{array}$ & 2.5 & $\begin{array}{l}2.5 \mathrm{~m} \text { barrel, } \\
\text { (G) }\end{array}$ & 1.00 & $\begin{array}{l}\text { decent penetration sand on } \\
\text { outside of core barrel }\end{array}$ \\
\hline
\end{tabular}

\begin{tabular}{|c|c|c|c|c|c|c|c|}
\hline \multicolumn{8}{|c|}{$\begin{array}{c}\text { J-2-90-SF } \\
\text { Carquinez Straits } \\
\text { Map K \& L }\end{array}$} \\
\hline 87 & SFB020690-1 & $\begin{array}{l}\text { On bank N. side of channel at } \\
\text { Benicia bend }\end{array}$ & $\begin{array}{r}38^{\circ} 2.45^{\prime} \\
-122^{\circ} 8.91^{\prime}\end{array}$ & 3 & $\begin{array}{l}1.75 \mathrm{~m} \text { barrel } \\
(\mathrm{G})\end{array}$ & .96 & gravel at the top \\
\hline 88 & SFB020690-2 & $\begin{array}{l}\text { On bank SE of South Hampton } \\
\text { Bay - off Commodore Jones } \\
\text { Point }\end{array}$ & $\begin{array}{r}38^{\circ} 3.32^{\prime} \\
-122^{\circ} 10.49^{\prime}\end{array}$ & 2.25 & $\begin{array}{l}2.5 \mathrm{~m} \text { barrel, } \\
(\mathrm{G})\end{array}$ & 2.30 & core nearly full \\
\hline 89 & SFB020690-3 & $\begin{array}{l}\text { On bank just off site } 2 \text { off } \\
\text { Commodore Jones Pt. }\end{array}$ & $\begin{array}{r}38^{\circ} 3.20^{\prime} \\
-122^{\circ} 10.42^{\prime}\end{array}$ & 5.4 & $\begin{array}{l}2.5 \mathrm{~m} \text { barrel, } \\
\text { (G) }\end{array}$ & 2.05 & $\begin{array}{l}\text { bury } 5 \text { core } \& \text { head top } 15 \mathrm{~cm} \\
\text { looks like detrital mud clasts } \\
\text { shells }\end{array}$ \\
\hline 90 & SFB020690-4 & Slightly deeper $>3$ & $\begin{array}{r}38^{\circ} 3.16^{\prime} \\
-122^{\circ} 10.53^{\prime}\end{array}$ & 7.5 & $\begin{array}{l}2.5 \mathrm{~m} \text { barrel, } \\
\text { (G) }\end{array}$ & 1.99 & less penetration shells at top \\
\hline 91 & SFB020690-5 & Deeper than $\# 90$ & $\begin{array}{r}38^{\circ} 3.21^{\prime} \\
-122^{\circ} 10.70^{\prime}\end{array}$ & 10.7 & $\begin{array}{l}1.5 \mathrm{~m} \text { barrel } \\
(\mathrm{G})\end{array}$ & .90 & barrel partly full \\
\hline 92 & SFB020690-06 & $\begin{array}{l}\text { Near base of slope smooth } \\
\text { bottom to } 18 \mathrm{~m}\end{array}$ & $\begin{array}{r}38^{\circ} 3.27^{\prime} \\
-122^{\circ} 10.59^{\prime}\end{array}$ & 12.75 & $\begin{array}{l}1.5 \mathrm{~m} \text { barrel } \\
(\mathrm{G})\end{array}$ & .63 & some sand \\
\hline
\end{tabular}




\begin{tabular}{|c|c|c|c|c|c|c|c|}
\hline $\begin{array}{l}\text { Map } \\
\#\end{array}$ & $\begin{array}{l}\text { Core } \\
\text { Number }\end{array}$ & Setting & $\begin{array}{l}\text { Lat. } \\
\text { Long. }\end{array}$ & $\begin{array}{l}\text { Water } \\
\text { Depth (m) }\end{array}$ & $\begin{array}{l}\text { Type } \\
\text { Core (G) } \\
\text { (B) }\end{array}$ & $\begin{array}{l}\text { Core } \\
\text { length } \\
(\mathrm{m})\end{array}$ & General Description \\
\hline
\end{tabular}

\section{J-2-90-SF}

San Pablo Bay from the mouth of the Napa River to Sisters Rocks

and Petaluma River

\begin{tabular}{|c|c|c|c|c|c|c|c|}
\hline \multicolumn{8}{|c|}{ Map J } \\
\hline 93 & SFB020690-7 & $\begin{array}{l}\text { On sediment build up in front } \\
\text { of dike } \# 9 \text {, Mare Island Strait }\end{array}$ & $\begin{array}{r}38^{\circ} 4.11^{\prime} \\
-122^{\circ} 14.14^{\prime}\end{array}$ & 4.75 & $\begin{array}{l}2.5 \mathrm{~m} \text { barrel, } \\
\text { (G) }\end{array}$ & 1.89 & sand and mud \\
\hline 94 & SFB020690-8 & $\begin{array}{l}\text { E. side of build up around pier } \\
\text { S.side }\end{array}$ & $\begin{array}{r}38^{\circ} 3.32^{\prime} \\
-122^{\circ} 15.29^{\prime} \\
\end{array}$ & 5 & & 1.68 & $52 \mathrm{~cm}$ of sand at top/mud \\
\hline 95 & SFB020690-9 & $\begin{array}{l}\text { Entrance to the Petaluma River } \\
\text { from San Pablo Bay-channel } \\
\text { floor }\end{array}$ & $\begin{array}{r}38^{\circ} 1.23^{\prime} \\
-122^{\circ} 25.68^{\prime}\end{array}$ & 3 & $\begin{array}{l}2.5 \mathrm{~m} \text { barrel, } \\
(\mathrm{G})\end{array}$ & 2.21 & \\
\hline 96 & SFB020690-10 & $\begin{array}{l}\text { W bank of Petaluma channel } \\
\text { mouth actually just beyond } \\
\text { bank top }\end{array}$ & $\begin{array}{r}38^{\circ} 2.66^{\prime} \\
-122^{\circ} 25.49^{\prime}\end{array}$ & 2.5 & $\begin{array}{l}2.5 \mathrm{~m} \text { barrel, } \\
(\mathrm{G})\end{array}$ & 2.50 & $\begin{array}{l}\text { slowly dropped, buried head at } \\
2.5 \text { m barrel }(G) \text { really soupy } \\
\text { mud }\end{array}$ \\
\hline 97 & SFB020690-11 & $\begin{array}{l}\text { Petaluma River off bend with } \\
\text { towers Black John slough, } \\
\text { accretionary bank-channel } \\
\text { floor is at } 4.5 \mathrm{~m}\end{array}$ & $\begin{array}{r}38^{\circ} 8.35^{\prime} \\
-122^{\circ} 31.06^{\prime}\end{array}$ & 3.5 & $\begin{array}{l}3.0 \mathrm{~m} \text { barrel } \\
(\mathrm{G})\end{array}$ & 2.84 & $\begin{array}{l}\text { buried barrel and head, mud no } \\
\text { shells at base }\end{array}$ \\
\hline 98 & SFB020690-12 & $\begin{array}{l}\text { W. margin of channel just } \\
\text { above base of channel }\end{array}$ & $\begin{array}{r}38^{\circ} 8.44^{\prime} \\
-122^{\circ} 31.04^{\prime} \\
\end{array}$ & 4 & $\begin{array}{l}3.0 \mathrm{~m} \text { barrel } \\
(\mathrm{G})\end{array}$ & 2.38 & \\
\hline 99 & SFB020690-13 & $\begin{array}{l}\text { Central San Pablo Bay N of } \\
\text { ship channel }\end{array}$ & $\begin{array}{r}38^{\circ} 2.38^{\prime} \\
-122^{\circ} 23.72^{\prime} \\
\end{array}$ & 2.5 & $\begin{array}{l}3.0 \mathrm{~m} \text { barrel } \\
(\mathrm{G})\end{array}$ & 2.89 & $\begin{array}{l}\text { smooth bottom full } 10 \text { ' core } \\
\text { but not extruded at top }\end{array}$ \\
\hline 100 & SFB020790-1 & San Pablo Bay & $\begin{array}{r}38^{\circ} 3.45^{\prime} \\
-122^{\circ} 16.26^{\prime} \\
\end{array}$ & 13 & $\begin{array}{l}2.5 \mathrm{~m} \text { barrel, } \\
(\mathrm{G})\end{array}$ & .39 & sandy clean sand/mud \\
\hline 101 & SFB020790-2 & $\begin{array}{l}\text { On bank (upper part) sand } \\
\text { waves at base }\end{array}$ & $\begin{array}{r}38^{\circ} 2.41^{\prime} \\
-122^{\circ} 16.71^{\prime} \\
\end{array}$ & 6.5 & $\begin{array}{l}2.5 \mathrm{~m} \text { barrel, } \\
(\mathrm{G})\end{array}$ & 1.90 & good core \\
\hline 102 & SFB020790-3 & $\begin{array}{l}\text { On old geochemical sample } \\
\text { site \#39 SW of Lone Tree Pt. } \\
\text { smooth bottom }\end{array}$ & $\begin{array}{r}38^{\circ} 1.83^{\prime} \\
-122^{\circ} 17.15^{\prime}\end{array}$ & & $\begin{array}{l}2.5 \mathrm{~m} \text { barrel, } \\
\text { (G) }\end{array}$ & 2.55 & shell at top \\
\hline 103 & SFB020790-4 & Pinole shoal & $\begin{array}{r}38^{\circ} 2.50^{\prime} \\
-122^{\circ} 18.92^{\prime} \\
\end{array}$ & 10 & $\begin{array}{l}2.5 \mathrm{~m} \text { barrel, } \\
(\mathrm{G})\end{array}$ & .97 & disturbed sand/mud \\
\hline 104 & SFB020790-5 & $\begin{array}{l}\text { N. side of ship channel San } \\
\text { Pablo Bay on platform }\end{array}$ & $\begin{array}{r}38^{\circ} 3.65^{\prime} \\
-122^{\circ} 19.69^{\prime}\end{array}$ & 4 & $\begin{array}{l}2.5 \mathrm{~m} \text { barrel } \\
(\mathrm{G})\end{array}$ & 2.03 & buried head, mud all the way \\
\hline
\end{tabular}




\begin{tabular}{|c|c|c|c|c|c|c|c|}
\hline $\begin{array}{l}\text { Map } \\
\#\end{array}$ & $\begin{array}{l}\text { Core } \\
\text { Number }\end{array}$ & Setting & $\begin{array}{l}\text { Lat. } \\
\text { Long. }\end{array}$ & $\begin{array}{l}\text { Water } \\
\text { Depth (m) }\end{array}$ & $\begin{array}{l}\text { Type } \\
\text { Core (G) } \\
\text { (B) } \\
\end{array}$ & $\begin{array}{l}\text { Core } \\
\text { length } \\
(\mathrm{m}) \\
\end{array}$ & General Description \\
\hline 105 & SFB020790-6 & $\begin{array}{l}\text { In } \sim 5.5 \mathrm{~m} \text { to test sand mud } \\
\text { interface } 5.5 \text { to } 6 \mathrm{~m} \text { sand } \\
\text { waves }\end{array}$ & $\begin{array}{r}38^{\circ} 3.03^{\prime} \\
-122^{\circ} 19.35^{\prime}\end{array}$ & $5.5-6$ & $\begin{array}{l}2.5 \mathrm{~m} \text { barrel, } \\
\text { (G) }\end{array}$ & 2.10 & $\begin{array}{l}\text { buried head no sand mud } \\
\text { throughout core split in barrel }\end{array}$ \\
\hline 106 & SFB020790-7 & $\begin{array}{l}\text { Old geochemical site \#45 in } \\
\text { shallow area N central San } \\
\text { Pablo Bay }\end{array}$ & $\begin{array}{r}38^{\circ} 4.40^{\prime} \\
-122^{\circ} 22.51^{\prime}\end{array}$ & 3.2 & $\begin{array}{l}3.0 \mathrm{~m} \text { barrel } \\
\text { (G) }\end{array}$ & & $\begin{array}{l}\text { undisturbed top oxidized mud } \\
\text { at surface }\end{array}$ \\
\hline 107 & SFB020790-8 & $\begin{array}{l}\text { S of ship channel, Pinole Shoal } \\
\text { middle part }\end{array}$ & $\begin{array}{r}38^{\circ} 2.02^{\prime} \\
-122^{\circ} 19.57^{\prime}\end{array}$ & 4.5 & $\begin{array}{l}2.5 \mathrm{~m} \text { barrel, } \\
(\mathrm{G})\end{array}$ & 2.38 & smooth bottom \\
\hline 108 & SFB020790-9 & Shallow central Pinole Shoal & $\begin{array}{r}38^{\circ} 1.61^{\prime} \\
-122^{\circ} 19.10^{\prime} \\
\end{array}$ & 3 & $\begin{array}{l}3.0 \mathrm{~m} \text { barrel } \\
(\mathrm{G})\end{array}$ & 2.53 & \\
\hline 109 & SFB020790-10 & $\begin{array}{l}\text { W end of Pinole Shoal } \\
\text { (Shallow) }\end{array}$ & $\begin{array}{r}38^{\circ} 1.19^{\prime} \\
-122^{\circ} 20.96^{\prime} \\
\end{array}$ & 3.5 & $\begin{array}{l}2.5 \mathrm{~m} \text { barrel, } \\
(\mathrm{G})\end{array}$ & 2.71 & shell lag \\
\hline 110 & SFB020790-11 & Off Pinole Pt, sloping bank & $\begin{array}{r}38^{\circ} 1.44^{\prime} \\
-122^{\circ} 22.09^{\prime} \\
\end{array}$ & 6 & $\begin{array}{l}2.5 \mathrm{~m} \text { barrel, } \\
(\mathrm{G})\end{array}$ & 1.79 & $\begin{array}{l}\text { sand waves on furrows on } \\
\text { fathometer }\end{array}$ \\
\hline 111 & SFB020790-12 & $\begin{array}{l}\text { W. of Pinole Pt. irregular } \\
\text { bottom }\end{array}$ & $\begin{array}{r}38^{\circ} 0.69^{\prime} \\
-122^{\circ} 22.53^{\prime} \\
\end{array}$ & 2 & $\begin{array}{l}2.5 \mathrm{~m} \text { barrel, } \\
\text { (G) }\end{array}$ & 2.49 & filled barrel \\
\hline 112 & SFB020790-13 & $\begin{array}{l}\text { Deeper water on slope between } \\
\text { Pt San Pablo at Pinole Pt, sand } \\
\text { waves }\end{array}$ & $\begin{array}{r}38^{\circ} 0.03^{\prime} \\
-122^{\circ} 23.70^{\prime}\end{array}$ & $\sim 5.2$ & $\begin{array}{l}2.5 \mathrm{~m} \text { barrel, } \\
(\mathrm{G})\end{array}$ & 2.03 & no sand waves \\
\hline 113 & SFB020790-14 & $\begin{array}{l}\text { Near bank top, NW of Pt San } \\
\text { Pablo (just on bank top) }\end{array}$ & $\begin{array}{r}37^{\circ} 59.35^{\prime} \\
-122^{\circ} 24.24^{\prime} \\
\end{array}$ & 3 & $\begin{array}{l}2.5 \mathrm{~m} \text { barrel, } \\
(\mathrm{G})\end{array}$ & 1.99 & sandy mud \\
\hline 114 & SFB020790-15 & $\begin{array}{l}\text { N. of Pt San Pedro in small } \\
\text { trough, very irregular bottom } 1 \\
\text { m relief, } \sim 3 \text { ' penetration }\end{array}$ & $\begin{array}{r}37^{\circ} 59.89^{\prime} \\
-122^{\circ} 26.58^{\prime}\end{array}$ & 5 & $\begin{array}{l}1.75 \mathrm{~m} \text { barrel } \\
(\mathrm{G})\end{array}$ & .93 & \\
\hline 115 & SFB020790-16 & $\begin{array}{l}\text { Old geochemical sample site } \\
\text { \#75, platform N. of Pt San } \\
\text { Pedro too shallow to reach }\end{array}$ & $\begin{array}{r}38^{\circ} 0.72^{\prime} \\
-122^{\circ} 27.04^{\prime}\end{array}$ & & $\begin{array}{l}3.0 \mathrm{~m} \text { barrel } \\
(\mathrm{G})\end{array}$ & 2.60 & good upper $65 \mathrm{~cm}$ shelly at top \\
\hline
\end{tabular}

\section{J-2-90-SF}

Richmond San Rafael Bay from

Pt. San Pedro to South of Paradise Cay Map J \& H

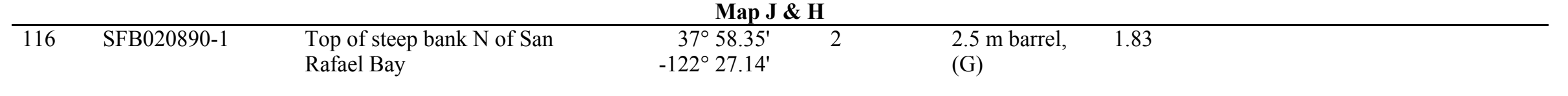




\begin{tabular}{|c|c|c|c|c|c|c|c|}
\hline $\begin{array}{l}\text { Мap } \\
\#\end{array}$ & $\begin{array}{l}\text { Core } \\
\text { Number }\end{array}$ & Setting & $\begin{array}{c}\text { Lat. } \\
\text { Long. }\end{array}$ & $\begin{array}{l}\text { Water } \\
\text { Depth (m) }\end{array}$ & $\begin{array}{l}\text { Type } \\
\text { Core (G) } \\
\text { (B) }\end{array}$ & $\begin{array}{l}\text { Core } \\
\text { length } \\
\text { (m) }\end{array}$ & General Description \\
\hline
\end{tabular}

\begin{tabular}{|c|c|c|c|c|c|c|c|}
\hline 117 & SFB020890-2 & Mid bank down from $\# 116$ & $\begin{array}{r}37^{\circ} 58.32^{\prime} \\
-122^{\circ} 27.00^{\prime} \\
\end{array}$ & 6.5 & $\begin{array}{l}2.5 \mathrm{~m} \text { barrel, } \\
(\mathrm{G})\end{array}$ & 2.10 & partly buried head \\
\hline 118 & SFB020890-3 & $\begin{array}{l}\text { Lower bank down from sites } \\
115 \& 116\end{array}$ & $\begin{array}{r}37^{\circ} 58.29^{\prime} \\
-122^{\circ} 26.86^{\prime} \\
\end{array}$ & $12 / 5$ & $\begin{array}{l}2.5 \mathrm{~m} \text { barrel, } \\
(\mathrm{G})\end{array}$ & 1.90 & good core not buried \\
\hline 119 & SFB020890-4 & $\begin{array}{l}\text { Channel floor down from } 115 \\
\& 116 \text { bottom flattens at } \sim \\
16.5 \mathrm{~m}\end{array}$ & $\begin{array}{r}37^{\circ} 58.17^{\prime} \\
-122^{\circ} 26.87^{\prime}\end{array}$ & 16.5 & $\begin{array}{l}2.5 \mathrm{~m} \text { barrel, } \\
\text { (G) }\end{array}$ & 1.47 & sandy at top \\
\hline 120 & SFB020890-5 & $\begin{array}{l}\text { Upper bank where it is more } \\
\text { gentle, } \mathrm{S} \text { of the San Rafael } \\
\text { boat channel, smooth bottom }\end{array}$ & $\begin{array}{r}37^{\circ} 57.69^{\prime} \\
-122^{\circ} 27.50^{\prime}\end{array}$ & 2.5 & $\begin{array}{l}3.0 \mathrm{~m} \text { barrel } \\
(\mathrm{G})\end{array}$ & 2.33 & buried head \\
\hline 121 & SFB020890-6 & $\begin{array}{l}\text { Down slope from } \# 120 \sim 5 \mathrm{~m} \\
\text { depth }\end{array}$ & $\begin{array}{r}37^{\circ} 57.61^{\prime} \\
-122^{\circ} 27.33^{\prime} \\
\end{array}$ & 5 & $\begin{array}{l}3.0 \mathrm{~m} \text { barrel } \\
(\mathrm{G})\end{array}$ & 2.32 & buried head \\
\hline 122 & SFB020890-7 & $\begin{array}{l}\text { Lower most slope below \#119 } \\
\& 120\end{array}$ & $\begin{array}{r}37^{\circ} 57.54^{\prime} \\
-122^{\circ} 27.15^{\prime}\end{array}$ & 13 & $\begin{array}{l}2.5 \mathrm{~m} \text { barrel, } \\
\text { (G) }\end{array}$ & 1.51 & \\
\hline 123 & SFB020890--8 & $\begin{array}{l}\text { Channel floor out from sites } \\
\# 119-121, \text { not quite flat }\end{array}$ & $\begin{array}{r}37^{\circ} 57.28^{\prime} \\
-122^{\circ} 26.68^{\prime}\end{array}$ & 19 & $\begin{array}{l}2.5 \mathrm{~m} \text { barrel, } \\
\text { (G) }\end{array}$ & 2.17 & sand/mud \\
\hline 124 & SFB020890-9 & $\begin{array}{l}\text { S. San Rafael Bridge or San } \\
\text { Quentin Bay platform at } 4 \mathrm{~m}\end{array}$ & $\begin{array}{r}37^{\circ} 55.43^{\prime} \\
-122^{\circ} 28.39^{\prime}\end{array}$ & 4 & $\begin{array}{l}3.0 \mathrm{~m} \text { barrel } \\
\text { (G) }\end{array}$ & 2.45 & $3.0 \mathrm{~m}$ barrel $(\mathrm{G})$ buried \\
\hline 125 & SFB020890-10 & On irregular bottom & $\begin{array}{r}37^{\circ} 54.78^{\prime} \\
-122^{\circ} 27.55^{\prime} \\
\end{array}$ & 7 & $\begin{array}{l}2.5 \mathrm{~m} \text { barrel, } \\
(\mathrm{G})\end{array}$ & 2.45 & buried \\
\hline 126 & SFB020890-11 & $\begin{array}{l}\text { Channel floor SE from } \\
\text { Paradise Cay }\end{array}$ & $\begin{array}{r}37^{\circ} 54.33^{\prime} \\
-122^{\circ} 27.60^{\prime} \\
\end{array}$ & 9 & $\begin{array}{l}2.5 \mathrm{~m} \text { barrel, } \\
(\mathrm{G})\end{array}$ & 1.45 & \\
\hline 127 & SFB020890-12 & $\begin{array}{l}\text { Inshore-up bank from depth } \\
5.5 \mathrm{~m}\end{array}$ & $\begin{array}{r}37^{\circ} 54.28^{\prime} \\
-122^{\circ} 27.91^{\prime} \\
\end{array}$ & 5.5 & $\begin{array}{l}2.5 \mathrm{~m} \text { barrel, } \\
(\mathrm{G})\end{array}$ & 1.90 & buried head \\
\hline 128 & SFB020890-13 & $\begin{array}{l}\text { Top of bank between Paradise } \\
\text { Cay and Pt Chauncey smooth } \\
\text { gently sloping bottom }\end{array}$ & $\begin{array}{r}37^{\circ} 54.17^{\prime} \\
-122^{\circ} 28.04^{\prime}\end{array}$ & 2 & $\begin{array}{l}2.5 \mathrm{~m} \text { barrel, } \\
\text { (G) }\end{array}$ & 2.49 & really buried head \\
\hline
\end{tabular}

\begin{tabular}{|c|c|c|c|c|c|c|c|}
\hline \multicolumn{8}{|c|}{$\begin{array}{c}\text { J-2-90-SF } \\
\text { Richardson Bay } \\
\text { Map H \& I } \\
\end{array}$} \\
\hline 129 & SFB020990-1 & Richardson Bay Center & $\begin{array}{r}37^{\circ} 52.46^{\prime} \\
-122^{\circ} 29.42^{\prime}\end{array}$ & 2 & $\begin{array}{l}3.0 \mathrm{~m} \text { barrel } \\
(\mathrm{G})\end{array}$ & 2.93 & mud \\
\hline 130 & SFB020990-2 & $\begin{array}{l}\text { Mouth of Richardson Bay } \\
\text { midway up gentle slope from } 4 \\
-2 \mathrm{~m} \text {, Upper part of a fairly } \\
\text { abrupt drop mud bottom }\end{array}$ & $\begin{array}{r}37^{\circ} 51.64^{\prime} \\
-122^{\circ} 28.19^{\prime}\end{array}$ & 5 & $\begin{array}{l}2.5 \mathrm{~m} \text { barrel, } \\
\text { (G) }\end{array}$ & 1.98 & lost a bit of the top of the core \\
\hline
\end{tabular}




\begin{tabular}{|c|c|c|c|c|c|c|c|}
\hline $\begin{array}{l}\text { Map } \\
\#\end{array}$ & $\begin{array}{l}\text { Core } \\
\text { Number }\end{array}$ & Setting & $\begin{array}{l}\text { Lat. } \\
\text { Long. }\end{array}$ & $\begin{array}{l}\text { Water } \\
\text { Depth (m) }\end{array}$ & $\begin{array}{l}\text { Type } \\
\text { Core (G) } \\
\text { (B) } \\
\end{array}$ & $\begin{array}{l}\text { Core } \\
\text { length } \\
(\mathrm{m}) \\
\end{array}$ & General Description \\
\hline 131 & SFB020990-3 & $\begin{array}{l}\text { Mouth of Richardson Bay, top } \\
\text { of steep slope into central bay }\end{array}$ & $\begin{array}{r}37^{\circ} 51.51^{\prime} \\
-122^{\circ} 28.15^{\prime}\end{array}$ & 6 & $\begin{array}{l}2.5 \mathrm{~m} \text { barrel, } \\
(\mathrm{G})\end{array}$ & 2.40 & $\begin{array}{l}\text { sand waves, mud, buried } 8^{\prime} \\
\text { core and head, shell in bottom, } \\
\text { sandy at the top }\end{array}$ \\
\hline 132 & SFB020990-4 & $\begin{array}{l}\text { Slope into Richardson Bay } \pm \\
\text { mid slope }\end{array}$ & $\begin{array}{r}37^{\circ} 51.40^{\prime} \\
-122^{\circ} 28.02^{\prime}\end{array}$ & 18.5 & $\begin{array}{l}2.5 \mathrm{~m} \text { barrel, } \\
(\mathrm{G})\end{array}$ & 2.08 & $\begin{array}{l}\text { mud buried to head double } \\
\text { reflector soupy top of core } \\
\text { transparent fluid layer. }\end{array}$ \\
\hline 133 & SFB020990-5 & Same location as \#131 & $\begin{array}{r}37^{\circ} 51.36^{\prime} \\
-122^{\circ} 28.00^{\prime}\end{array}$ & 18.5 & $\begin{array}{l}1.75 \mathrm{~m} \text { barrel } \\
\text { (G) }\end{array}$ & & $\begin{array}{l}\text { upper part of sedimentary } \\
\text { column good core yogurt like } \\
\text { mud at top, potters clay at base }\end{array}$ \\
\hline 134 & SFB020990-6 & $\begin{array}{l}\text { Still further down slope from } \\
\# 131 \text { and } 1321 \mathrm{~m} \text { above the } \\
\text { base of the slope }\end{array}$ & $\begin{array}{r}37^{\circ} 51.29^{\prime} \\
-122^{\circ} 28.08^{\prime}\end{array}$ & 29 & $\begin{array}{l}2.5 \mathrm{~m} \text { barrel, } \\
(\mathrm{G})\end{array}$ & 2.50 & $\begin{array}{l}\text { mud buried head full } 8 \text { ' core } \\
\text { intact top }\end{array}$ \\
\hline
\end{tabular}

\section{J-2-90-SF}

Central Bay Off Richmond and Berkeley Marinas

Map I \& H

\begin{tabular}{|c|c|c|c|c|c|c|c|}
\hline 135 & SFB020990-7 & $\begin{array}{l}\text { A few meters down slope from } \\
\# 133 \text { to sample area below } \\
\text { break in slope }\end{array}$ & $\begin{array}{r}37^{\circ} 51.22^{\prime} \\
-122^{\circ} 28.06^{\prime}\end{array}$ & 30 & $\begin{array}{l}2.5 \mathrm{~m} \text { barrel, } \\
\text { (G) }\end{array}$ & $\mathrm{N} / \mathrm{C}$ & $\begin{array}{l}\text { Muddy shell sand, gravel bag } \\
\text { sample sand/gravel/soft mud } \\
\text { slight penetration }\end{array}$ \\
\hline 136 & SFB020990-8 & $\begin{array}{l}\text { Crest of South Hampton Shoal } \\
\pm \text { irregular bottom with flank }{ }^{ \pm} \\
\text {smooth top }\end{array}$ & $\begin{array}{r}37^{\circ} 53.05^{\prime} \\
-122^{\circ} 24.00^{\prime}\end{array}$ & 7 & $\begin{array}{l}1.75 \mathrm{~m} \text { barrel } \\
\text { (G) }\end{array}$ & .20 & some penetration sand \\
\hline 137 & SFB020990-9 & $\begin{array}{l}\text { Trough floor E of South } \\
\text { Hampton Shoal }\end{array}$ & $\begin{array}{r}37^{\circ} 53.30^{\prime} \\
-122^{\circ} 23.65^{\prime}\end{array}$ & 8 & $\begin{array}{l}2.5 \mathrm{~m} \text { barrel, } \\
\text { (G) }\end{array}$ & 1.30 & sandy mud \\
\hline 138 & SFB020990-10 & $\begin{array}{l}\text { Sloping bank WSW of Brooks } \\
\text { Island }\end{array}$ & $\begin{array}{r}37^{\circ} 53.59^{\prime} \\
-122^{\circ} 23.25^{\prime} \\
\end{array}$ & 4 & & & \\
\hline 139 & SFB020990-11 & $\begin{array}{l}\text { Shallow subtidal flats S. of } \\
\text { Richmond }\end{array}$ & $\begin{array}{r}37^{\circ} 53.37^{\prime} \\
-122^{\circ} 21.47^{\prime} \\
\end{array}$ & 2 & $\begin{array}{l}3.0 \mathrm{~m} \text { barrel } \\
(\mathrm{G})\end{array}$ & filled & \\
\hline 140 & SFB020990-12 & $\begin{array}{l}\text { Platform } \mathrm{N} \text { of Berkeley Pier } \\
\text { distinct bottom irregular sand } \\
\text { waves }\end{array}$ & $\begin{array}{r}37^{\circ} 51.41^{\prime} \\
-122^{\circ} 21.54^{\prime}\end{array}$ & 4.5 & $\begin{array}{l}3.0 \mathrm{~m} \text { barrel } \\
\text { (G) }\end{array}$ & 2.04 & mud \\
\hline 141 & SFB020990-13 & $\begin{array}{l}\text { On platform } \mathrm{N} \text { of Treasure } \\
\text { Island }\end{array}$ & $\begin{array}{r}37^{\circ} 50.87^{\prime} \\
-122^{\circ} 22.67^{\prime}\end{array}$ & 9.5 & $\begin{array}{l}3.0 \mathrm{~m} \text { barrel } \\
(\mathrm{G})\end{array}$ & & poor penetration sticky sand \\
\hline
\end{tabular}




\begin{tabular}{|c|c|c|c|c|c|c|c|}
\hline $\begin{array}{l}\text { Map } \\
\#\end{array}$ & $\begin{array}{l}\text { Core } \\
\text { Number }\end{array}$ & Setting & $\begin{array}{l}\text { Lat. } \\
\text { Long. }\end{array}$ & $\begin{array}{l}\text { Water } \\
\text { Depth (m) }\end{array}$ & $\begin{array}{l}\text { Type } \\
\text { Core (G) } \\
\text { (B) }\end{array}$ & $\begin{array}{l}\text { Core } \\
\text { length } \\
(\mathrm{m})\end{array}$ & General Description \\
\hline
\end{tabular}

\section{J-2-90-SF}

South San Francisco Bay

South and adjacent to the San Mateo Bridge

\begin{tabular}{|c|c|c|c|c|c|c|c|}
\hline 142 & SFB021290-1 & $\begin{array}{l}\text { Above top of bank } \sim 1.2 \mathrm{~m} \mathrm{~S} \\
\text { of San Mateo Bridge }\end{array}$ & $\begin{array}{r}37^{\circ} 34.03^{\prime} \\
-122^{\circ} 14.10^{\prime}\end{array}$ & 3 & $\begin{array}{l}3.0 \mathrm{~m} \text { barrel } \\
(\mathrm{G})\end{array}$ & 2.34 & shell on top \\
\hline 143 & SFB021290-2 & $\begin{array}{l}\text { Top of bank just above break } \\
\text { in slope }\end{array}$ & $\begin{array}{r}37^{\circ} 34.12^{\prime} \\
-122^{\circ} 14.03^{\prime} \\
\end{array}$ & & $\begin{array}{l}3.0 \mathrm{~m} \text { barrel } \\
(\mathrm{G})\end{array}$ & 2.06 & shell at top and bottom \\
\hline 144 & SFB021290-3 & Bank slope & $\begin{array}{r}37^{\circ} 34.11^{\prime} \\
-122^{\circ} 13.83^{\prime}\end{array}$ & 6.65 & $\begin{array}{l}3.0 \mathrm{~m} \text { barrel } \\
(\mathrm{G})\end{array}$ & 1.57 & shell at top and bottom \\
\hline 145 & SFB021290-4 & Base of slope & $\begin{array}{r}37^{\circ} 34.24^{\prime} \\
-122^{\circ} 13.96^{\prime}\end{array}$ & 10.5 & $\begin{array}{l}3.0 \mathrm{~m} \text { barrel } \\
(\mathrm{G})\end{array}$ & 1.38 & \\
\hline 146 & SFB021290-5 & Mid channel & $\begin{array}{r}37^{\circ} 34.35^{\prime} \\
-122^{\circ} 13.60^{\prime}\end{array}$ & 14.5 & $\begin{array}{l}3.0 \mathrm{~m} \text { barrel } \\
\text { (G) }\end{array}$ & 1.18 & Shell at surface sand at depth \\
\hline 147 & SFB021290-6 & Above slope top & $\begin{array}{r}37^{\circ} 34.80^{\prime} \\
-122^{\circ} 13.38^{\prime}\end{array}$ & 4 & $\begin{array}{l}3.0 \mathrm{~m} \text { barrel } \\
\text { (G) }\end{array}$ & 1.49 & shell at top \\
\hline 148 & SFB021290-7 & Upper mid-bank slope & $\begin{array}{r}37^{\circ} 34.47^{\prime} \\
-122^{\circ} 13.29^{\prime} \\
\end{array}$ & 5.75 & $\begin{array}{l}3.0 \mathrm{~m} \text { barrel } \\
(\mathrm{G})\end{array}$ & 2.21 & \\
\hline 149 & SFB021290-8 & Base of slope & $\begin{array}{r}37^{\circ} 34.55^{\prime} \\
-122^{\circ} 13.26^{\prime} \\
\end{array}$ & 16 & $\begin{array}{l}3.0 \mathrm{~m} \text { barrel } \\
(\mathrm{G})\end{array}$ & 1.76 & stiff mud at bottom \\
\hline 150 & SFB021290-9 & Lower slope bank & $\begin{array}{r}37^{\circ} 34.63^{\prime} \\
-122^{\circ} 13.27^{\prime} \\
\end{array}$ & 10.5 & $\begin{array}{l}3.0 \mathrm{~m} \text { barrel } \\
(\mathrm{G})\end{array}$ & & Stiff mud at base \\
\hline 151 & SFB021290-10 & $\begin{array}{l}\text { Shallow subtidal flats SE of } \\
\text { San Mateo Bridge }\end{array}$ & $\begin{array}{r}37^{\circ} 34.80^{\prime} \\
-122^{\circ} 11.57^{\prime} \\
\end{array}$ & 3.5 & $\begin{array}{l}3.0 \mathrm{~m} \text { barrel } \\
(\mathrm{G})\end{array}$ & & \\
\hline
\end{tabular}

\section{J-2-90-SF}

South San Francisco Bay North of the San Mateo Bridge

to Potrero Point and Alameda outer harbor

\begin{tabular}{|c|c|c|c|c|c|c|}
\hline \multicolumn{7}{|c|}{ Map G, F \& B } \\
\hline 152 & SFB021290-11 & $\begin{array}{l}\text { Shallow subtidal flats off } \\
\text { Coyote Pt. }\end{array}$ & $\begin{array}{r}37^{\circ} 35.63^{\prime} \\
-122^{\circ} 18.94^{\prime}\end{array}$ & 3.5 & $\begin{array}{l}3.0 \mathrm{~m} \text { barrel } \\
\text { (G) }\end{array}$ & buried head \\
\hline 153 & SFB021290-12 & $\begin{array}{l}\text { Base of slope } \mathrm{N} \text { of } \# 8 \text { Marker } \\
\text { at or near the slope base }\end{array}$ & $\begin{array}{r}37^{\circ} 36.63^{\prime} \\
-122^{\circ} 17.67^{\prime}\end{array}$ & 16 & $\begin{array}{l}3.0 \mathrm{~m} \text { barrel } \\
\text { (G) }\end{array}$ & \\
\hline
\end{tabular}




\begin{tabular}{|c|c|c|c|c|c|c|c|}
\hline $\begin{array}{l}\text { Мap } \\
\#\end{array}$ & $\begin{array}{l}\text { Core } \\
\text { Number }\end{array}$ & Setting & $\begin{array}{c}\text { Lat. } \\
\text { Long. }\end{array}$ & $\begin{array}{l}\text { Water } \\
\text { Depth (m) }\end{array}$ & $\begin{array}{l}\text { Type } \\
\text { Core (G) } \\
\text { (B) }\end{array}$ & $\begin{array}{l}\text { Core } \\
\text { length } \\
\text { (m) }\end{array}$ & General Description \\
\hline
\end{tabular}

\begin{tabular}{|c|c|c|c|c|c|c|c|}
\hline 154 & SFB021590-1 & $\begin{array}{l}\text { Top of slope just } \mathrm{N} \text { of } \# 8 \mathrm{~N} \text { of } \\
\text { San Mateo Bridge }\end{array}$ & $\begin{array}{r}37^{\circ} 36.61^{\prime} \\
-122^{\circ} 17.96^{\prime} \\
\end{array}$ & 3.25 & $\begin{array}{l}2.5 \mathrm{~m} \text { barrel } \\
(\mathrm{G})\end{array}$ & 1.95 & $\begin{array}{l}\text { shelly at surface, shell layer at } \\
\text { bottom }\end{array}$ \\
\hline 155 & SFB021590-2 & Mid-slope $\mathrm{N}$ of $\# 8$ & $\begin{array}{r}37^{\circ} 36.69^{\prime} \\
-122^{\circ} 17.89^{\prime} \\
\end{array}$ & 7 & $\begin{array}{l}2.5 \mathrm{~m} \text { barrel } \\
(\mathrm{G})\end{array}$ & 2.51 & $\begin{array}{l}\text { penetrate to core top shell at } \\
\text { top and bottom of core }\end{array}$ \\
\hline 156 & SFB021590-3 & $\begin{array}{l}\text { W. bank top off SFO ship } \\
\text { channel }\end{array}$ & $\begin{array}{r}37^{\circ} 37.83^{\prime} \\
-122^{\circ} 19.56^{\prime}\end{array}$ & 3.8 & $\begin{array}{l}2.5 \mathrm{~m} \text { barrel } \\
(\mathrm{G})\end{array}$ & 2.25 & bury head \\
\hline 157 & SFB021590-4 & Base of slope E of \#3 & $\begin{array}{r}37^{\circ} 37.90^{\prime} \\
-122^{\circ} 19.26^{\prime} \\
\end{array}$ & 8 & $\begin{array}{l}2.5 \mathrm{~m} \text { barrel } \\
(\mathrm{G})\end{array}$ & 2.35 & $\begin{array}{l}\text { bury head shell layers in } \\
\text { bottom }\end{array}$ \\
\hline 158 & SFB021590-5 & $\begin{array}{l}\text { Mid channel S of ship channel } \\
\text { furrow field }\end{array}$ & $\begin{array}{r}37^{\circ} 38.28^{\prime} \\
-122^{\circ} 18.67^{\prime} \\
\end{array}$ & 10.5 & $\begin{array}{l}2.5 \mathrm{~m} \text { barrel } \\
(\mathrm{G})\end{array}$ & 2.13 & shelly at base \\
\hline 159 & SFB021590-6 & $\begin{array}{l}\text { Subtidal flats off SFO, flat } \\
\text { bottom }\end{array}$ & $\begin{array}{r}37^{\circ} 37.91^{\prime} \\
-122^{\circ} 20.33^{\prime}\end{array}$ & 4 & & 2.48 & shell at top \\
\hline 160 & SFB021590-7 & Channel floor off Oyster Pt. & $\begin{array}{r}37^{\circ} 39.55^{\prime} \\
-122^{\circ} 20.82^{\prime} \\
\end{array}$ & 6 & $\begin{array}{l}2.5 \mathrm{~m} \text { barrel } \\
(\mathrm{G})\end{array}$ & & sink head \\
\hline 161 & SFB021590-8 & $\begin{array}{l}\text { Top of bank S of Hunters } \\
\text { Point }\end{array}$ & $\begin{array}{r}37^{\circ} 41.83^{\prime} \\
-122^{\circ} 22.16^{\prime}\end{array}$ & 1.8 & $\begin{array}{l}3.0 \mathrm{~m} \text { barrel } \\
(\mathrm{G})\end{array}$ & 2.69 & \\
\hline 162 & SFB021590-9 & Mid-bank E of \#8 & $\begin{array}{r}37^{\circ} 41.82^{\prime} \\
-122^{\circ} 22.03^{\prime}\end{array}$ & 4.5 & $\begin{array}{l}3.0 \mathrm{~m} \text { barrel } \\
(\mathrm{G})\end{array}$ & 2.55 & buried barrel and head \\
\hline 163 & SFB021590-10 & $\begin{array}{l}\text { Base of slope E of \#160 and } \\
161\end{array}$ & $\begin{array}{r}37^{\circ} 41.82^{\prime} \\
-122^{\circ} 21.88^{\prime}\end{array}$ & 6.25 & & 2.63 & \\
\hline 164 & SFB021590-11 & $\begin{array}{l}\text { Channel floor off Hunter s } \\
\text { Point }\end{array}$ & $\begin{array}{r}37^{\circ} 42.59^{\prime} \\
-122^{\circ} 21.06^{\prime} \\
\end{array}$ & 13 & $\begin{array}{l}3.0 \mathrm{~m} \text { barrel } \\
(\mathrm{G})\end{array}$ & & $\begin{array}{l}\text { full penetration lost of shell on } \\
\text { anchor }\end{array}$ \\
\hline 165 & SFB021590-12 & Channel off Pt Advisadero & $\begin{array}{r}37^{\circ} 43.36^{\prime} \\
-122^{\circ} 19.73^{\prime} \\
\end{array}$ & & $\begin{array}{l}1.75 \mathrm{~m} \text { barrel } \\
(\mathrm{G})\end{array}$ & 1.17 & \\
\hline 166 & SFB021590-13 & Channel off Potrero point & $\begin{array}{r}37^{\circ} 45.09^{\prime} \\
-122^{\circ} 20.09^{\prime} \\
\end{array}$ & 14 & $\begin{array}{l}1.75 \mathrm{~m} \text { barrel } \\
(\mathrm{G})\end{array}$ & & very short core sand \\
\hline 167 & SFB021590-14 & $\begin{array}{l}\text { S. of Alameda evenly gently } \\
\text { sloping bottom } 30^{\prime}-6 \text { in } \\
\text { general transition of sand and } \\
\text { mud }\end{array}$ & $\begin{array}{r}37^{\circ} 45.44^{\prime} \\
-122^{\circ} 18.79^{\prime}\end{array}$ & 6 & $\begin{array}{l}2.5 \mathrm{~m} \text { barrel } \\
(\mathrm{G})\end{array}$ & 2.52 & $\begin{array}{l}\text { filled with sandy mud, shell at } \\
\text { top }\end{array}$ \\
\hline 168 & SFB021590-15 & $\begin{array}{l}\text { Shoal area } \mathrm{S} \text { of Alameda } \\
\text { smooth bottom }\end{array}$ & $\begin{array}{r}37^{\circ} 45.25^{\prime} \\
-122^{\circ} 17.42^{\prime} \\
\end{array}$ & 2.5 & $\begin{array}{l}2.5 \mathrm{~m} \text { barrel } \\
(\mathrm{G})\end{array}$ & 2.68 & shell at top and bottom of core \\
\hline 169 & SFB021590-16 & $\begin{array}{l}\text { In burrow areas in bottom of } \\
\text { one of the excavations normal } \\
\text { depth } 3.5\end{array}$ & $\begin{array}{r}37^{\circ} 43.91^{\prime} \\
-122^{\circ} 17.02^{\prime}\end{array}$ & 10 & $\begin{array}{l}2.5 \mathrm{~m} \text { barrel } \\
(\mathrm{G})\end{array}$ & 1.90 & $\begin{array}{l}\text { bury barrel and head stiff mud } \\
\text { in core cutter }\end{array}$ \\
\hline
\end{tabular}




\begin{tabular}{|c|c|c|c|c|c|c|c|}
\hline $\begin{array}{c}\text { Map } \\
\#\end{array}$ & $\begin{array}{l}\text { Core } \\
\text { Number }\end{array}$ & Setting & $\begin{array}{l}\text { Lat. } \\
\text { Long. }\end{array}$ & $\begin{array}{l}\text { Water } \\
\text { Depth (m) }\end{array}$ & $\begin{array}{l}\text { Type } \\
\text { Core (G) } \\
\text { (B) } \\
\end{array}$ & \begin{tabular}{|l|} 
Core \\
length \\
$(\mathrm{m})$ \\
\end{tabular} & General Description \\
\hline 170 & SFB021590-17 & $\begin{array}{l}\text { Subtidal flat off N. tip of } \\
\text { Oakland Airport }\end{array}$ & $\begin{array}{r}37^{\circ} 43.43^{\prime} \\
-122^{\circ} 16.48^{\prime} \\
\end{array}$ & 4 & $\begin{array}{l}2.5 \mathrm{~m} \text { barrel } \\
(\mathrm{G})\end{array}$ & 2.91 & \\
\hline 171 & SFB021590-18 & $\begin{array}{l}\text { Just S. of patch of sand waves } \\
\text { on smooth bottom }\end{array}$ & $\begin{array}{r}37^{\circ} 43.01^{\prime} \\
-122^{\circ} 16.97^{\prime} \\
\end{array}$ & 4 & $\begin{array}{l}2.5 \mathrm{~m} \text { barrel } \\
(\mathrm{G})\end{array}$ & .65 & shell/ mud \\
\hline 172 & SFB021590-19 & $\begin{array}{l}\text { Near base of gentle slope off } \\
\text { NW end Oakland Airport }\end{array}$ & $\begin{array}{r}37^{\circ} 42.26^{\prime} \\
-122^{\circ} 16.65^{\prime} \\
\end{array}$ & & $\begin{array}{l}2.5 \mathrm{~m} \text { barrel } \\
(\mathrm{G})\end{array}$ & 2.52 & \\
\hline 173 & SFB021590-20 & Crest of San Bruno shoal & $\begin{array}{r}37^{\circ} 39.94^{\prime} \\
-122^{\circ} 18.20^{\prime} \\
\end{array}$ & 4 & $\begin{array}{l}1.75 \mathrm{~m} \text { barrel } \\
(\mathrm{G})\end{array}$ & & $\begin{array}{l}\text { some penetration, sandy } \\
\text { w/shells }\end{array}$ \\
\hline 174 & SFB022090-1 & $\begin{array}{l}\text { Area on E. side of channel N. } \\
\text { of San Mateo Bridge up to } \\
\text { South Hampton Shoal bank } \\
\text { top, flat bottom }\end{array}$ & $\begin{array}{r}37^{\circ} 36.15^{\prime} \\
-122^{\circ} 15.51^{\prime}\end{array}$ & 4.5 & $\begin{array}{l}3.0 \mathrm{~m} \text { barrel } \\
(\mathrm{G})\end{array}$ & 2.58 & $\begin{array}{l}\text { core bottoms in mixed clean } \\
\text { sand, mud penetrates to barrel } \\
\text { top }\end{array}$ \\
\hline 175 & SFB022090-2 & $\begin{array}{l}\text { In topographically irregular } \\
\text { area }\end{array}$ & $\begin{array}{r}37^{\circ} 36.01^{\prime} \\
-122^{\circ} 15.57^{\prime} \\
\end{array}$ & 5 & $\begin{array}{l}3.0 \mathrm{~m} \text { barrel } \\
(\mathrm{G})\end{array}$ & 2.63 & hint of shell layer in bottom \\
\hline 176 & SFB022090-3 & $\begin{array}{l}\text { Mid-slope of bank E. side of } \\
\text { channel }\end{array}$ & $\begin{array}{r}37^{\circ} 35.90^{\prime} \\
-122^{\circ} 15.68^{\prime} \\
\end{array}$ & 10 & $\begin{array}{l}3.0 \mathrm{~m} \text { barrel } \\
(\mathrm{G})\end{array}$ & 2.44 & stiff green clay at base \\
\hline 177 & SFB022090-4 & Lower slope of E. bank & $\begin{array}{r}37^{\circ} 35.73^{\prime} \\
-122^{\circ} 15.61^{\prime} \\
\end{array}$ & 14 & $\begin{array}{l}3.0 \mathrm{~m} \text { barrel } \\
(\mathrm{G})\end{array}$ & 2.14 & $\begin{array}{l}\text { green-yellow stiff clay at core } \\
\text { base, shelly at top. }\end{array}$ \\
\hline 178 & SFB022090-5 & Channel floor in furrow field & $\begin{array}{r}37^{\circ} 35.53^{\prime} \\
-122^{\circ} 15.86^{\prime} \\
\end{array}$ & 17 & $\begin{array}{l}3.0 \mathrm{~m} \text { barrel } \\
(\mathrm{G})\end{array}$ & 2.17 & $\begin{array}{l}\text { furrows evident good } \\
\text { stratification }\end{array}$ \\
\hline 179 & SFB022090-6 & $\begin{array}{l}\text { Above abrupt scarp (dredge) } \\
\text { N. of bridge }\end{array}$ & $\begin{array}{r}37^{\circ} 36.45^{\prime} \\
-122^{\circ} 15.21^{\prime} \\
\end{array}$ & 3.5 & $\begin{array}{l}3.0 \mathrm{~m} \text { barrel } \\
(\mathrm{G})\end{array}$ & 2.50 & lots of shells on anchor \\
\hline 180 & SFB022090-7 & Below break in slope & $\begin{array}{r}37^{\circ} 36.37^{\prime} \\
-122^{\circ} 15.34^{\prime}\end{array}$ & & $\begin{array}{l}3.0 \mathrm{~m} \text { barrel } \\
\text { (G) }\end{array}$ & & $\begin{array}{l}\text { buried } 3.0 \mathrm{~m} \text { barrel }(\mathrm{G}) \text { and } \\
\text { head, bag the material from the } \\
\text { core cutter.? }\end{array}$ \\
\hline 181 & SFB022090-8A & $\begin{array}{l}\text { In area w/ topographic } \\
\text { irregularities on bottom } \sim 0.5 \\
\text { m relief }\end{array}$ & $\begin{array}{r}37^{\circ} 37.61^{\prime} \\
-122^{\circ} 16.08^{\prime}\end{array}$ & 5.5 & $\begin{array}{l}1.75 \mathrm{~m} \text { barrel } \\
(\mathrm{G})\end{array}$ & $\begin{array}{l}\text { filled } \\
\text { barrel }\end{array}$ & filled $1.75 \mathrm{~m}$ barrel $(\mathrm{G})$ \\
\hline 182 & SFB022090-8 & In same area as $\# 180$ & $\begin{array}{r}37^{\circ} 37.70^{\prime} \\
-122^{\circ} 16.24^{\prime} \\
\end{array}$ & 5.5 & $\begin{array}{l}2.5 \mathrm{~m} \text { barrel } \\
(\mathrm{G})\end{array}$ & 1.81 & shelly at top over mud \\
\hline 183 & SFB022090-9 & $\begin{array}{l}\text { Mound structure SE from S. } \\
\text { crest of San Bruno Shoal }\end{array}$ & $\begin{array}{r}37^{\circ} 38.37^{\prime} \\
-122^{\circ} 16.59^{\prime} \\
\end{array}$ & 5.5 & $\begin{array}{l}3.0 \mathrm{~m} \text { barrel } \\
(\mathrm{G})\end{array}$ & 2.43 & \\
\hline 184 & SFB022090-10 & $\begin{array}{l}\text { Subtidal flats W of San } \\
\text { Lorenzo Creek }\end{array}$ & $\begin{array}{r}37^{\circ} 40.46^{\prime} \\
-122^{\circ} 14.66^{\prime}\end{array}$ & 3.5 & $\begin{array}{l}3.0 \mathrm{~m} \text { barrel } \\
\text { (G) }\end{array}$ & 2.65 & \\
\hline
\end{tabular}




\begin{tabular}{|c|c|c|c|c|c|c|c|}
\hline Мap & $\begin{array}{l}\text { Core } \\
\text { Number }\end{array}$ & Setting & $\begin{array}{l}\text { Lat. } \\
\text { Long. }\end{array}$ & $\begin{array}{l}\text { Water } \\
\text { Depth (m) }\end{array}$ & $\begin{array}{l}\text { Type } \\
\text { Core (G) } \\
\text { (B) } \\
\end{array}$ & $\begin{array}{l}\text { Core } \\
\text { length } \\
(\mathrm{m}) \\
\end{array}$ & General Description \\
\hline 185 & SFB022090-11 & $\begin{array}{l}1^{\text {st }} \text { appearance of bottom } \\
\text { irregularities on shallow } \\
\text { subtidal flat E of S. Lorenzo }\end{array}$ & $\begin{array}{r}37^{\circ} 41.28^{\prime} \\
-122^{\circ} 15.48^{\prime}\end{array}$ & & $\begin{array}{l}3.0 \mathrm{~m} \text { barrel } \\
\text { (G) }\end{array}$ & & $\begin{array}{l}\text { Pipeline area - core in flat } \\
\text { bottom just beyond dredged } \\
\text { material }\end{array}$ \\
\hline 186 & SFB022090-12 & Bottom irregularities neutral & $\begin{array}{r}37^{\circ} 41.37^{\prime} \\
-122^{\circ} 16.05^{\prime} \\
\end{array}$ & 4.5 & $\begin{array}{l}3.0 \mathrm{~m} \text { barrel } \\
(\mathrm{G})\end{array}$ & & \\
\hline 187 & SFB022090-13 & Low ridge $\mathrm{w} / \mathrm{sand}$ waves & $\begin{array}{r}37^{\circ} 41.82^{\prime} \\
-122^{\circ} 16.91^{\prime} \\
\end{array}$ & 4.5 & $\begin{array}{l}3.0 \mathrm{~m} \text { barrel } \\
(\mathrm{G})\end{array}$ & & $\begin{array}{l}\text { not likely sand waves/ bury } \\
1.25 \mathrm{~m} \text { barrel }(\mathrm{G}) \mathrm{s}\end{array}$ \\
\hline 188 & SFB022090-14 & $\begin{array}{l}\text { Trough of E of N end San } \\
\text { Bruno Shoal }\end{array}$ & $\begin{array}{r}37^{\circ} 41.38^{\prime} \\
-122^{\circ} 18.13^{\prime} \\
\end{array}$ & 9.25 & $\begin{array}{l}3.0 \mathrm{~m} \text { barrel } \\
(\mathrm{G})\end{array}$ & short core & sand at bottom \\
\hline 189 & SFB022090-15 & $\begin{array}{l}\text { Trough E of San Bruno Shoals } \\
\text { upper end }\end{array}$ & $\begin{array}{r}37^{\circ} 39.27^{\prime} \\
-122^{\circ} 17.00^{\prime} \\
\end{array}$ & 5.5 & $\begin{array}{l}3.0 \mathrm{~m} \text { barrel } \\
(\mathrm{G})\end{array}$ & 2.32 & \\
\hline 190 & SFB022090-16 & $\begin{array}{l}\text { In mined area at S. end of San } \\
\text { Bruno Shoal- on natural flay } \\
\text { (finger that protrudes } \mathrm{N} \text { in the } \\
\text { middle of dredged hole) }\end{array}$ & $\begin{array}{r}37^{\circ} 37.80^{\prime} \\
-122^{\circ} 17.17^{\prime}\end{array}$ & 3 & $\begin{array}{l}3.0 \mathrm{~m} \text { barrel } \\
(\mathrm{G})\end{array}$ & 2.64 & sandy mud \\
\hline 191 & SFB022090-17 & $\begin{array}{l}\text { On bench at } 6.5 \mathrm{~m} \text { within } \\
\text { excavation }\end{array}$ & $\begin{array}{r}37^{\circ} 37.91^{\prime} \\
-122^{\circ} 17.41^{\prime} \\
\end{array}$ & 6.5 & $\begin{array}{l}3.0 \mathrm{~m} \text { barrel } \\
(\mathrm{G})\end{array}$ & & shell at bottom of core \\
\hline 192 & SFB022090-18 & At bottom of excavation & $\begin{array}{r}37^{\circ} 37.84^{\prime} \\
-122^{\circ} 17.57^{\prime} \\
\end{array}$ & 10 & $\begin{array}{l}3.0 \mathrm{~m} \text { barrel } \\
(\mathrm{G})\end{array}$ & 2.21 & all mud \\
\hline 193 & SFB02190-1MH & $\begin{array}{l}\text { Slump area off Newark } \\
\text { Slough., On slope in evacuated } \\
\text { zone of slump }\end{array}$ & $\begin{array}{r}37^{\circ} 29.76^{\prime} \\
-122^{\circ} 5.29^{\prime}\end{array}$ & 5 & $\begin{array}{l}1.75 \mathrm{~m} \text { barrel } \\
\text { (G) }\end{array}$ & 1.27 & Core for geotechnical analysis \\
\hline 194 & SFB02190-1A & Same area as $\# 192$ & $\begin{array}{r}37^{\circ} 29.76^{\prime} \\
-122^{\circ} 5.29^{\prime} \\
\end{array}$ & 5 & $\begin{array}{l}1.75 \mathrm{~m} \text { barrel } \\
(\mathrm{G})\end{array}$ & 1.19 & \\
\hline 195 & SFB022190-1 & $\begin{array}{l}\text { Free fall in same area of cores } \\
192-193\end{array}$ & $\begin{array}{r}37^{\circ} 29.76^{\prime} \\
-122^{\circ} 5.29^{\prime} \\
\end{array}$ & 5 & $\begin{array}{l}3.0 \mathrm{~m} \text { barrel } \\
(\mathrm{G})\end{array}$ & 1.80 & free fall \\
\hline 196 & $\begin{array}{l}\text { SFB022190- } \\
2 \mathrm{MH}\end{array}$ & Above slump mass & $\begin{array}{r}37^{\circ} 29.84^{\prime} \\
-122^{\circ} 5.55^{\prime} \\
\end{array}$ & $3.25-3.5$ & $\begin{array}{l}2.5 \mathrm{~m} \text { barrel } \\
(\mathrm{G})\end{array}$ & 1.71 & $\begin{array}{l}\text { Core for geotechnical analysis } \\
1^{\text {st }} \text { free fall }\end{array}$ \\
\hline 197 & SFB022190-2 & Same area as 195 & $\begin{array}{r}37^{\circ} 29.84^{\prime} \\
-122^{\circ} 5.55^{\prime} \\
\end{array}$ & $3.25-3.50$ & $\begin{array}{l}2.5 \mathrm{~m} \text { barrel } \\
(\mathrm{G})\end{array}$ & 1.73 & \\
\hline 198 & $\begin{array}{l}\text { SFB022190- } \\
3 \mathrm{MH}\end{array}$ & $\begin{array}{l}\text { North edge of irregular } \\
\text { bottom, down slope from } 194 \\
\text { and } 195 \text { in slump mass }\end{array}$ & $\begin{array}{r}37^{\circ} 29.84^{\prime} \\
-122^{\circ} 5.74^{\prime}\end{array}$ & 7.5 & $\begin{array}{l}2.5 \mathrm{~m} \text { barrel } \\
(\mathrm{G})\end{array}$ & 1.58 & $\begin{array}{l}\text { Core for geotechnical analysis } \\
\text { free fall, upper } 1.5^{\prime} \text { probably } \\
\text { disturbed }\end{array}$ \\
\hline 199 & SFB0221903A & Same area as 197 & $\begin{array}{r}37^{\circ} 29.84^{\prime} \\
-122^{\circ} 5.74^{\prime} \\
\end{array}$ & 7.5 & $\begin{array}{l}1.75 \mathrm{~m} \text { barrel } \\
(\mathrm{G})\end{array}$ & 1.40 & power down full \\
\hline
\end{tabular}




\begin{tabular}{|c|c|c|c|c|c|c|c|}
\hline $\begin{array}{l}\text { Map } \\
\#\end{array}$ & $\begin{array}{l}\text { Core } \\
\text { Number }\end{array}$ & Setting & $\begin{array}{l}\text { Lat. } \\
\text { Long. }\end{array}$ & $\begin{array}{l}\text { Water } \\
\text { Depth (m) }\end{array}$ & $\begin{array}{l}\text { Type } \\
\text { Core (G) } \\
\text { (B) } \\
\end{array}$ & $\begin{array}{l}\text { Core } \\
\text { length } \\
\text { (m) } \\
\end{array}$ & General Description \\
\hline 200 & SFB022190-4 & Base of slope, toe of slump & $\begin{array}{r}37^{\circ} 29.66^{\prime} \\
-122^{\circ} 5.53^{\prime}\end{array}$ & 10 & $\begin{array}{l}2.5 \mathrm{~m} \text { barrel } \\
(\mathrm{G})\end{array}$ & 2.05 & $\begin{array}{l}\text { power down all the way to } \\
\text { bottom }\end{array}$ \\
\hline
\end{tabular}




\begin{tabular}{|c|c|c|c|c|c|c|c|}
\hline $\begin{array}{l}\text { Мap } \\
\#\end{array}$ & $\begin{array}{l}\text { Core } \\
\text { Number }\end{array}$ & Setting & $\begin{array}{c}\text { Lat. } \\
\text { Long. }\end{array}$ & $\begin{array}{l}\text { Water } \\
\text { Depth (m) }\end{array}$ & $\begin{array}{l}\text { Type } \\
\text { Core (G) } \\
\text { (B) }\end{array}$ & $\begin{array}{l}\text { Core } \\
\text { length } \\
\text { (m) }\end{array}$ & General Description \\
\hline
\end{tabular}

\section{J-1-91-SF}

1991 Cores, January and February 1991

Gravity and Drill Cores

Sacramento to the mouth of the San Joaquin River

\begin{tabular}{|c|c|c|c|c|c|c|c|}
\hline \multicolumn{8}{|c|}{ Map O } \\
\hline 1 & $012391-1$ & $\begin{array}{l}\text { N. side Sac. River above }(\mathrm{N}) \\
\text { on Dredged channel }\end{array}$ & $\begin{array}{r}38^{\circ} 6.23^{\prime} \\
-121^{\circ} 43.14^{\prime} \\
\end{array}$ & 3.5 & $\begin{array}{l}1.25 \mathrm{~m} \text { barrel } \\
(\mathrm{G})\end{array}$ & $.53 \mathrm{~m}$ & $\begin{array}{l}\text { sandy in upper part live } \\
\text { bivalves at top }\end{array}$ \\
\hline 2 & $012391-2$ & $\begin{array}{l}\text { Sac. River under E most power } \\
\text { line relocation of site } \\
15(1 / 31 / 90)\end{array}$ & $\begin{array}{r}38^{\circ} 5.15^{\prime} \\
-121^{\circ} 44.98^{\prime}\end{array}$ & 6 & $\begin{array}{l}1.25 \mathrm{~m} \text { barrel } \\
(\mathrm{G})\end{array}$ & $1.3 \mathrm{~m}$ & $\begin{array}{l}\text { has organic detritus layer lose } \\
\text { at least } 5 \mathrm{~cm} \text { at the top }\end{array}$ \\
\hline 3 & $012391-3$ & Sac. River same site as \#2 & $\begin{array}{r}38^{\circ} 5.15^{\prime} \\
-121^{\circ} 44.98^{\prime}\end{array}$ & 6 & $\begin{array}{l}2.5 \mathrm{~m} \text { barrel } \\
(\mathrm{G})\end{array}$ & 2.10 & $\begin{array}{l}\text { peaty detrital layer, sandy at } \\
\text { base sample }\end{array}$ \\
\hline 4 & $012391-4$ & $\begin{array}{l}\text { Sac. River at } 9 \mathrm{~m} \mathrm{H}_{2} \mathrm{O} \mathrm{N} \text {. side } \\
\text { of shipping channel bank } 0.11- \\
>\mathrm{N} \text { bank, under } \mathrm{E} \text { line of } \\
\text { double power line }\end{array}$ & $\begin{array}{r}38^{\circ} 4.90^{\prime} \\
-121^{\circ} 45.29^{\prime}\end{array}$ & 9 & $\begin{array}{l}2.5 \mathrm{~m} \text { barrel } \\
(\mathrm{G})\end{array}$ & 1.08 & $\begin{array}{l}\text { get partial core sandy mud no } \\
\text { organics at the base }\end{array}$ \\
\hline 5 & $012391-5$ & $\begin{array}{l}\text { Sac. River slightly shallower } \\
\text { than site } 50.06->\mathrm{N} \text { bank. }\end{array}$ & $\begin{array}{r}38^{\circ} 4.91^{\prime} \\
-121^{\circ} 45.29^{\prime} \\
\end{array}$ & 7.9 & $\begin{array}{l}2.5 \mathrm{~m} \text { barrel } \\
(\mathrm{G})\end{array}$ & 2.20 & peat at base sample collected \\
\hline 6 & 012391-6 & $\begin{array}{l}\text { Sac. River N. bank of channel } \\
\text { above dredged channel }\end{array}$ & $\begin{array}{r}38^{\circ} 4.55^{\prime} \\
-121^{\circ} 46.08^{\prime}\end{array}$ & 6.8 & $\begin{array}{l}2.5 \mathrm{~m} \text { barrel } \\
(\mathrm{G})\end{array}$ & 2.25 & $\begin{array}{l}\text { bury } 1.25 \mathrm{~m} \text { barrel }(\mathrm{G}) \text { lose top } \\
\text { try again } \mathrm{w} / 10^{\prime} \text { detrital peat at } \\
\text { base again-sample }\end{array}$ \\
\hline 7 & $012391-7$ & $\begin{array}{l}\text { Sac. River just down slope into } \\
\text { channel } \\
0.15->\text { n. bank, .29->\#131. 23- } \\
>\# 12\end{array}$ & $\begin{array}{r}38^{\circ} 4.52^{\prime} \\
-121^{\circ} 45.97^{\prime}\end{array}$ & 9.3 & $\begin{array}{l}2.5 \mathrm{~m} \text { barrel } \\
(\mathrm{G})\end{array}$ & .70 & sand on core barrel \\
\hline 8 & $012391-8$ & $\begin{array}{l}\text { Sac. River split difference be- } \\
\text { tween } 11 \& 12 \text { since they were } \\
\text { so different. }\end{array}$ & $\begin{array}{r}38^{\circ} 4.41^{\prime} \\
-121^{\circ} 45.92^{\prime}\end{array}$ & 8 & $2.5 \mathrm{~m}$ barrel $(\mathrm{G})$ & 1.78 & peat at the base \\
\hline
\end{tabular}




\begin{tabular}{|c|c|c|c|c|c|c|c|}
\hline $\begin{array}{l}\text { Map } \\
\#\end{array}$ & $\begin{array}{l}\text { Core } \\
\text { Number }\end{array}$ & Setting & $\begin{array}{l}\text { Lat. } \\
\text { Long. }\end{array}$ & $\begin{array}{l}\text { Water } \\
\text { Depth (m) }\end{array}$ & $\begin{array}{l}\text { Type } \\
\text { Core (G) } \\
\text { (B) } \\
\end{array}$ & $\begin{array}{l}\text { Core } \\
\text { length } \\
(\mathrm{m}) \\
\end{array}$ & General Description \\
\hline 9 & $012391-9$ & $\begin{array}{l}\text { San Joaquin River try to core } \\
\text { on isolated sand ridges east } \\
\text { of\#12 core attempt on high } \\
\text { feature }\end{array}$ & $\begin{array}{r}38^{\circ} 3.82^{\prime} \\
-121^{\circ} 47.40^{\prime}\end{array}$ & 6.0 & $2.5 \mathrm{~m}$ barrel $(\mathrm{G})$ & .72 & coarse clean sand/mud \\
\hline 10 & $012391-10$ & $\begin{array}{l}\text { San Joaquin River 1.12->\#2, } \\
.86->\# 1, .06->\text { E. Bank }\end{array}$ & $\begin{array}{r}38^{\circ} 3.74^{\prime} \\
-121^{\circ} 47.29^{\prime}\end{array}$ & - & $2.5 \mathrm{~m}$ barrel $(\mathrm{G})$ & .22 & Coarse sand \\
\hline
\end{tabular}

\begin{tabular}{|c|c|c|c|c|c|c|}
\hline \multirow[b]{2}{*}{11} & \multicolumn{6}{|c|}{$\begin{array}{l}\text { J-1-91-SF } \\
\text { Suisun Bay to Benicia Bridge } \\
\text { Map M }\end{array}$} \\
\hline & $012491-1$ & $\begin{array}{l}\text { Suisun Slough near mouth flat, } \\
\text { smooth bottom in channel axis }\end{array}$ & $\begin{array}{r}38^{\circ} 8.47^{\prime} \\
-122^{\circ} 4.00^{\prime}\end{array}$ & 5.4 & $2.5 \mathrm{~m}$ barrel $(\mathrm{G}) \quad 1.95$ & wood detritus at base \\
\hline 12 & $012491-2$ & $\begin{array}{l}\text { Suisun Slough along bank next } \\
\text { to mouth }\end{array}$ & $\begin{array}{r}38^{\circ} 8.71^{\prime} \\
-122^{\circ} 4.05^{\prime} \\
\end{array}$ & 2.5 & $2.5 \mathrm{~m}$ barrel $(\mathrm{G}) 2.10$ & upper part really disturbed \\
\hline 13 & $012491-3$ & $\begin{array}{l}\text { Suisun Bay near sites } 9 \& 10 \\
\text { at Grizzly Bay mouth }(2 / 1 / 90)\end{array}$ & $\begin{array}{r}38^{\circ} 6.33^{\prime} \\
-122^{\circ} 2.95^{\prime}\end{array}$ & & $2.5 \mathrm{~m}$ barrel $(\mathrm{G}) .33$ & eased in filled to win 5 in. \\
\hline 14 & $012491-4$ & $\begin{array}{l}\text { Suisun Bay in thalweg, .87 -> } \\
\# 9, .84->\text { dol., . } 49->\text { Garnett Pt } \\
.99->\text { Pt Buckley }\end{array}$ & $\begin{aligned} 38^{\circ} & 6.20^{\prime} \\
-122^{\circ} & 1.93^{\prime}\end{aligned}$ & 3.5 & $2.5 \mathrm{~m}$ barrel $(\mathrm{G}) \quad 1.17$ & sand in lower part \\
\hline 15 & $012491-5$ & $\begin{array}{l}\text { Suisun Bay on slope more like } \\
\text { site } 8 \text { last yr., .97->\#9, .86- } \\
>\text { dol., .87->pt. Buckley. } \\
.51->\text { Garnett Pt. }\end{array}$ & $\begin{aligned} 38^{\circ} & 6.17^{\prime} \\
-122^{\circ} & 1.71^{\prime}\end{aligned}$ & 3.4 & $2.5 \mathrm{~m}$ barrel $(\mathrm{G}) 2.08$ & no wood at base \\
\hline 16 & $012491-6$ & $\begin{array}{l}\text { nearer to } 8, .72->\text { Pt. Buckley } \\
.92->\text { dol. } 1.13->\# 9\end{array}$ & $\begin{array}{r}38^{\circ} 6.05^{\prime} \\
-122^{\circ} 1.42^{\prime} \\
\end{array}$ & 3.4 & $2.5 \mathrm{~m}$ barrel $(\mathrm{G}) 2.41$ & no wood at base \\
\hline 17 & $012491-7$ & $\begin{array}{l}\mathrm{NE} \text { of } \mathrm{N} \text { end of Ryer Island, } \\
1.20->\# 90, .40->\text { shore } \\
2.38->\# 6\end{array}$ & $\begin{array}{r}38^{\circ} 5.82^{\prime} \\
-122^{\circ} 2.21^{\prime}\end{array}$ & 3.5 & $2.5 \mathrm{~m}$ barrel $(\mathrm{G}) \quad .90$ & organics, shells \\
\hline 18 & $012491-8$ & $\begin{array}{l}\text { Next to SW corner of Ryer } \\
\text { Island, .84->platform, } \\
.6->\text { shore, 1.09->R20, } \\
2.00->25\end{array}$ & $\begin{array}{r}38^{\circ} 4.67^{\prime} \\
-121^{\circ} 59.64^{\prime}\end{array}$ & $\sim 2$ & $2.5 \mathrm{~m}$ barrel $(\mathrm{G}) \quad 1.31$ & $\begin{array}{l}\text { 40 cm penetration eased, } \\
\text { dropped good core sand } / \text { mud }\end{array}$ \\
\hline
\end{tabular}




\begin{tabular}{|c|c|c|c|c|c|c|c|}
\hline $\begin{array}{l}\text { Map } \\
\#\end{array}$ & $\begin{array}{l}\text { Core } \\
\text { Number }\end{array}$ & Setting & $\begin{array}{c}\text { Lat. } \\
\text { Long. }\end{array}$ & $\begin{array}{l}\text { Water } \\
\text { Depth (m) }\end{array}$ & $\begin{array}{l}\text { Type } \\
\text { Core (G) } \\
\text { (B) } \\
\end{array}$ & $\begin{array}{l}\text { Core } \\
\text { length } \\
(\mathrm{m}) \\
\end{array}$ & General Description \\
\hline 19 & $012491-9$ & $\begin{array}{l}\text { Suisun Bay SW corner of Ryer } \\
\text { Island.42->dolphin NW of } \\
\text { Honker Bay, .57->Pt. Palo } \\
\text { Alto, .88->\#24A, 1.10->\#21 }\end{array}$ & $\begin{array}{r}38^{\circ} 4.10^{\prime} \\
-121^{\circ} 57.30^{\prime}\end{array}$ & - & $2.5 \mathrm{~m}$ barrel $(\mathrm{G})$ & 1.01 & - \\
\hline
\end{tabular}

\section{J-1-91-SF}

San Joaquin River to the confluence with the Sacramento

\begin{tabular}{|c|c|c|c|c|c|c|c|}
\hline \multicolumn{8}{|c|}{ Map N } \\
\hline 20 & $012591-1$ & $\begin{array}{l}\text { Zigzag structures, .49->\#1 Sac. } \\
\text { R., 1.57->\# Broad Slough } \\
.06->\text { W. Bank }\end{array}$ & $\begin{array}{r}38^{\circ} 3.43^{\prime} \\
-121^{\circ} 50.79^{\prime}\end{array}$ & 7 & $2.5 \mathrm{~m}$ barrel $(\mathrm{G})$ & .95 & Sand/ mud \\
\hline 21 & $012591-2$ & $\begin{array}{l}\text { N.side of Fraser Shoal, } \\
.49->\# 1 \text { Sac..R., .67->\#5 Sac. } \\
\text { R., 1.57->\#1 Broad Slough }\end{array}$ & $\begin{array}{r}38^{\circ} 3.50^{\prime} \\
-121^{\circ} 50.39^{\prime}\end{array}$ & 3 & Grab & bag & \\
\hline 22 & $012591-3$ & $\begin{array}{l}\text { W. side Fraser Shoal.75->\#3 } \\
\text { Sac. R.1.26->\#1 Broad } \\
\text { Slough.32->W. Bank }\end{array}$ & $\begin{array}{r}38^{\circ} 3.05^{\prime} \\
-121^{\circ} 50.34^{\prime}\end{array}$ & 3 & $2.5 \mathrm{~m}$ barrel $(\mathrm{G})$ & 1.13 & Sand at top \\
\hline 23 & $012591-4$ & $\begin{array}{l}\text { San Joaquin River grab W. } \\
\text { side of Fraser Shoal, .3->N\#1, } \\
.28->\text { E.bank, .31->\#1 }\end{array}$ & $\begin{array}{r}38^{\circ} 2.32^{\prime} \\
-121^{\circ} 49.99^{\prime}\end{array}$ & 3 & Grab & - & sand \\
\hline 24 & $012591-5$ & $\begin{array}{l}\text { San Joaquin River grab sand } \\
\text { waves just off \#1 in channel, } \\
.16->\# 1, .62->\# 4, .31->\# 2\end{array}$ & $\begin{array}{r}38^{\circ} 2.11^{\prime} \\
-121^{\circ} 49.81^{\prime}\end{array}$ & 5.5 & Grab & - & sand well sorted \\
\hline 25 & $012591-6$ & $\begin{array}{l}\text { San Joaquin River off Beener } \\
\text { Pt in sand wave field in Broad } \\
\text { Slough, .19->\#1, .55->\#4 }\end{array}$ & $\begin{array}{r}38^{\circ} 1.90^{\prime} \\
-121^{\circ} 49.96^{\prime}\end{array}$ & $7.5-8.09$ & Grab & - & sand waves \\
\hline 26 & $012591-7$ & $\begin{array}{l}\text { Main San Joaquin Channel big } \\
\text { sand waves; .47->\#4,.43->\#1, } \\
.18->\# 11\end{array}$ & $\begin{array}{r}38^{\circ} 1.66^{\prime} \\
-121^{\circ} 50.29^{\prime}\end{array}$ & 13 & Grab & - & coarse sand \\
\hline
\end{tabular}




\begin{tabular}{|c|c|c|c|c|c|c|c|}
\hline $\begin{array}{l}\text { Map } \\
\#\end{array}$ & $\begin{array}{l}\text { Core } \\
\text { Number }\end{array}$ & Setting & $\begin{array}{c}\text { Lat. } \\
\text { Long. }\end{array}$ & $\begin{array}{l}\text { Water } \\
\text { Depth (m) }\end{array}$ & $\begin{array}{l}\text { Type } \\
\text { Core (G) } \\
\text { (B) }\end{array}$ & $\begin{array}{l}\text { Core } \\
\text { length } \\
(\mathrm{m})\end{array}$ & General Description \\
\hline
\end{tabular}

\begin{tabular}{|c|c|c|c|c|c|c|c|}
\hline \multicolumn{8}{|c|}{$\begin{array}{l}\text { J-1-91-SF } \\
\text { Suisun Bay } \\
\text { Map M }\end{array}$} \\
\hline 27 & 013191-1 & $\begin{array}{l}\text { Suisun Bay at end of side scan } \\
\text { run; } .67->\# 9,1.52->\# 6 \text {, } \\
.87->N \text {. Dol, } .68->N \text { bank } \\
.96->\text { Garnett pt. }\end{array}$ & $\begin{array}{r}38^{\circ} 5.82^{\prime} \\
-122^{\circ} 3.28^{\prime}\end{array}$ & 7.5 & $2.5 \mathrm{~m}$ barrel $(\mathrm{G})$ & $1.11 \mathrm{~m}$ & \\
\hline 28 & $013191-2$ & $\begin{array}{l}\text { Suisun Bay vicinity of site } 12 \\
(2 / 1 / 90) \text { to look for wood layer } \\
\text { in areas of similar depth } \\
\text { nearby- follow } 10^{\prime} \text { isobath } \\
\text { S.W. of \#6 to } \sim .3 \mathrm{~nm} \text { from } \# 6 \\
.39->\# 6, .77->\# 4, .67->\mathrm{S} \\
\text { dolphin, } 1.37->\mathrm{N} \text {. dolphin }\end{array}$ & $\begin{array}{r}38^{\circ} 4.57^{\prime} \\
-122^{\circ} 4.93^{\prime}\end{array}$ & 4.5 & $2.5 \mathrm{~m}$ barrel $(\mathrm{G})$ & 1.14 & sand at top \\
\hline 29 & 013191-3 & $\begin{array}{l}\text { Suisun Bay just up channel } \\
\text { from; \#1, 2.49->\#6, .73->\#4, } \\
1.37->\text { N.dolphin }\end{array}$ & $\begin{array}{r}38^{\circ} 4.54^{\prime} \\
-122^{\circ} 4.65^{\prime}\end{array}$ & 2.8 & $2.5 \mathrm{~m}$ barrel $(\mathrm{G})$ & 1.29 & Sand/mud \\
\hline 30 & 013191-4 & $\begin{array}{l}\text { Suisun Bay off N.W. end Ryer } \\
\text { Island; } .34 \mathrm{~nm}->\text { each of the } \\
\text { two points on Ryer Isl., .32-> } \\
\text { S most Pt., 1.32->\#9, } \\
.86->\text { Dolphin }\end{array}$ & $\begin{array}{r}38^{\circ} 5.24^{\prime} \\
-122^{\circ} 2.60^{\prime}\end{array}$ & 2.0 & $2.5 \mathrm{~m}$ barrel $(\mathrm{G})$ & 1.30 & buried the head \\
\hline 31 & $013191-5$ & $\begin{array}{l}\text { Suisun Bay same locality as } \\
\# 31 \text { use } 3.0 \mathrm{~m} \text { barrel }(\mathrm{G})\end{array}$ & $\begin{array}{r}38^{\circ} 5.24^{\prime} \\
-122^{\circ} 2.62^{\prime} \\
\end{array}$ & 2.0 & $2.5 \mathrm{~m}$ barrel $(\mathrm{G})$ & 2.21 & organics at base \\
\hline 32 & 013191-6 & $\begin{array}{l}\text { Suisun Bay channel bank S.W. } \\
\text { of Ryer Island; .45->platform, } \\
.98->\text { N. dolphin } 1.96->\# 9\end{array}$ & $\begin{array}{r}38^{\circ} 4.84^{\prime} \\
-122^{\circ} 2.19^{\prime}\end{array}$ & 4.5 & $2.5 \mathrm{~m}$ barrel $(\mathrm{G})$ & 1.73 & \\
\hline 33 & 013191-7 & $\begin{array}{l}\text { Suisun Bay off E. end Roe Is. } \\
\text { (Gillespie Pt.) mud slope }\end{array}$ & $\begin{array}{r}38^{\circ} 4.12^{\prime} \\
-122^{\circ} 0.62^{\prime}\end{array}$ & 4.5 & $2.5 \mathrm{~m}$ barrel $(\mathrm{G})$ & .38 & $\begin{array}{l}1.25 \mathrm{~m} \text { barrel }(\mathrm{G}) \text { dropped } \\
\text { good penetration did not bury } \\
\text { head. }\end{array}$ \\
\hline
\end{tabular}




\begin{tabular}{|c|c|c|c|c|c|c|c|}
\hline $\begin{array}{c}\text { Мap } \\
\#\end{array}$ & $\begin{array}{l}\text { Core } \\
\text { Number }\end{array}$ & Setting & $\begin{array}{c}\text { Lat. } \\
\text { Long. }\end{array}$ & $\begin{array}{l}\text { Water } \\
\text { Depth (m) }\end{array}$ & $\begin{array}{l}\text { Type } \\
\text { Core (G) } \\
\text { (B) }\end{array}$ & $\begin{array}{l}\text { Core } \\
\text { length } \\
\text { (m) }\end{array}$ & General Description \\
\hline
\end{tabular}

\begin{tabular}{|c|c|c|c|c|c|c|}
\hline \multirow[b]{2}{*}{34} & \multicolumn{6}{|c|}{$\begin{array}{c}\text { J-2-91-SF } \\
\text { Carquinez Straits } \\
\text { Map L }\end{array}$} \\
\hline & $020191-1$ & $\begin{array}{l}\text { Carquinez Straits off } \\
\text { Commodore Jones Pt.; } \\
.93->21 \text { Dillon Pt } \\
.21->\text { Commodore Jones Pt. } \\
.50->\# 22\end{array}$ & $\begin{array}{r}38^{\circ} 3.29^{\prime} \\
-122^{\circ} 10.55^{\prime}\end{array}$ & $.4 \mathrm{~m}$ & $2.5 \mathrm{~m}$ barrel $(\mathrm{G}) \quad 1.33$ & $1.25 \mathrm{~m}$ barrel $(\mathrm{G})$ eased in full \\
\hline 35 & $020191-2$ & $\begin{array}{l}\text { Carquinez Straits West of } \# 23 \\
\text { Benicia Pt .21->\#23 } \\
.74->\# 22,1.14->\text { T cross }\end{array}$ & $\begin{array}{r}38^{\circ} 2.60^{\prime} \\
-122^{\circ} 10.12^{\prime}\end{array}$ & 2.6 & $2.5 \mathrm{~m}$ barrel $(\mathrm{G}) \quad 1.39$ & $\begin{array}{l}1.25 \mathrm{~m} \text { barrel }(\mathrm{G}) \text { eased in full } \\
\text { barrel }\end{array}$ \\
\hline 36 & $020191-3$ & $\begin{array}{l}\text { Same location as \#35 used } 3.0 \\
\mathrm{~m} \text { barrel }(\mathrm{G})\end{array}$ & $\begin{array}{r}38^{\circ} 2.60^{\prime} \\
-122^{\circ} 10.12^{\prime} \\
\end{array}$ & 2.6 & $3.0 \mathrm{~m}$ barrel $(\mathrm{G}) 2.55$ & used $3.0 \mathrm{~m}$ barrel $(\mathrm{G})$ \\
\hline 37 & $020191-4$ & $\begin{array}{l}\text { Carquinez Straits } 1 / 2 \text { of the } \\
\text { way down bank .12->\#23 } \\
.75->\# 22,1.04->\text { T. cross }\end{array}$ & $\begin{array}{r}38^{\circ} 2.72^{\prime} \\
-122^{\circ} 10.28^{\prime}\end{array}$ & 10.5 & $2.5 \mathrm{~m}$ barrel $(\mathrm{G})$ & $\begin{array}{l}1.25 \mathrm{~m} \text { barrel }(\mathrm{G}) \text { eased in, } \\
\text { full, organics at base in well } \\
\text { defined layers }\end{array}$ \\
\hline 38 & $020191-5$ & $\begin{array}{l}\text { Carquinez Straits same } \\
\text { location as \#37 }\end{array}$ & $\begin{array}{r}38^{\circ} 2.70^{\prime} \\
-122^{\circ} 9.97^{\prime} \\
\end{array}$ & 10.5 & $2.5 \mathrm{~m}$ barrel $(\mathrm{G}) \quad 2.40$ & $3.0 \mathrm{~m}$ barrel $(\mathrm{G})$ dropped \\
\hline 39 & $020191-6$ & $\begin{array}{l}\text { Carquinez Straits East of Ozol } \\
\text { Dock on mud bank; } \\
.54->\text { T-cross, } .52->\text { w. end } \\
\text { jetty Marina, .16-> shore }\end{array}$ & $\begin{array}{r}38^{\circ} 2.84^{\prime} \\
-122^{\circ} 10.09^{\prime}\end{array}$ & 1.75 & $2.5 \mathrm{~m}$ barrel $(\mathrm{G}) 2.27$ & $3.0 \mathrm{~m}$ barrel $(\mathrm{G})$ buried head \\
\hline
\end{tabular}

\section{J-2-91-SF}

San Pablo Bay

Map J

\begin{tabular}{|c|c|c|c|c|c|c|}
\hline 40 & 020691-1 & $\begin{array}{l}\text { Central San Pablo Bay;.18->\#1 } \\
.31->\# 2\end{array}$ & $\begin{array}{l}38^{\circ} 0.99^{\prime} \\
-122^{\circ} 25.71^{\prime} \\
\end{array}$ & 2.5 & $2.5 \mathrm{~m}$ barrel $(\mathrm{G}) \quad 1.33$ & $1.25 \mathrm{~m}$ barrel $(\mathrm{G})$ eased in \\
\hline 41 & $020691-2$ & $\begin{array}{l}\text { N.E. central San Pablo Bay; } \\
.84->\# 9,1.36->\# 7,1.61->\# 11\end{array}$ & $\begin{array}{l}38^{\circ} 3.14^{\prime} \\
-122^{\circ} 22.18^{\prime} \\
\end{array}$ & 2.1 & $2.5 \mathrm{~m}$ barrel $(\mathrm{G}) 2.42$ & $\begin{array}{l}3.0 \mathrm{~m} \text { barrel }(\mathrm{G}) \text { short drop } \\
\text { kept upright plugged top }\end{array}$ \\
\hline 42 & $020691-3$ & $\begin{array}{l}\text { San Pablo Bay mid bank N. } \\
\text { side Pinole channel .49->\#9, } \\
1.41->\# 1\end{array}$ & $\begin{array}{l}38^{\circ} 2.91^{\prime} \\
-122^{\circ} 21.87^{\prime}\end{array}$ & 5.1 & $2.5 \mathrm{~m}$ barrel $(\mathrm{G}) 2.42$ & - \\
\hline
\end{tabular}




\begin{tabular}{|c|c|c|c|c|c|c|c|}
\hline $\begin{array}{l}\text { Map } \\
\#\end{array}$ & $\begin{array}{l}\text { Core } \\
\text { Number }\end{array}$ & Setting & $\begin{array}{l}\text { Lat. } \\
\text { Long. }\end{array}$ & $\begin{array}{l}\text { Water } \\
\text { Depth (m) }\end{array}$ & $\begin{array}{l}\text { Type } \\
\text { Core (G) } \\
\text { (B) } \\
\end{array}$ & $\begin{array}{l}\text { Core } \\
\text { length } \\
\text { (m) }\end{array}$ & General Description \\
\hline 43 & 020691-4 & $\begin{array}{l}\text { San Pablo Bay lower bank N. } \\
\text { side of Pinole channel near \#9, } \\
\text { irregular bottom; .14->\#9, } \\
1.20->\# 7,1.27->\# 11\end{array}$ & $\begin{array}{l}38^{\circ} 2.62^{\prime} \\
-122^{\circ} 21.45^{\prime}\end{array}$ & - & $2.5 \mathrm{~m}$ barrel $(\mathrm{G})$ & 1.89 & $\begin{array}{l}\text { Interbedded clean sand } / \mathrm{mud} \\
\text { top } 50 \mathrm{~cm}\end{array}$ \\
\hline 44 & $020691-5$ & $\begin{array}{l}\text { San Pablo Bay, .96->\#5, } \\
2.00->\# 1,1.49->\text { Sisters }\end{array}$ & $\begin{array}{l}38^{\circ} 0.51^{\prime} \\
-122^{\circ} 26.65^{\prime}\end{array}$ & 3.9 & $3.0 \mathrm{~m}$ barrel $(\mathrm{G})$ & 2.54 & mud sandy upper $40-50 \mathrm{~cm}$. \\
\hline
\end{tabular}

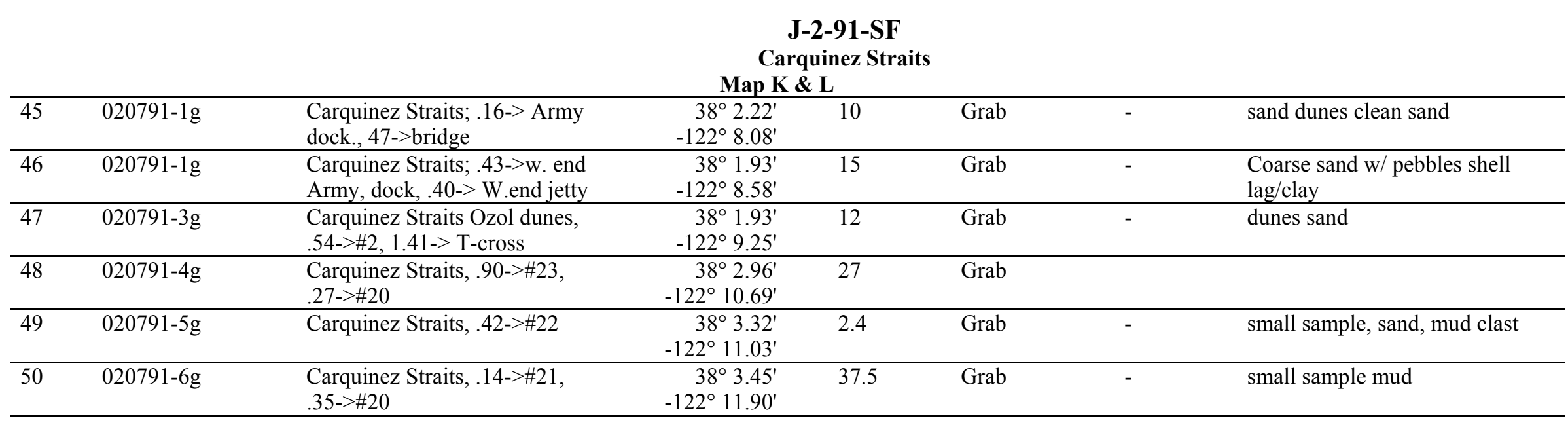

\begin{tabular}{|c|c|c|c|c|c|c|}
\hline \multicolumn{7}{|c|}{$\begin{array}{c}\text { J-2-91-SF } \\
\text { San Pablo Bay } \\
\text { Map J }\end{array}$} \\
\hline 51 & 020791-1 & $\begin{array}{l}\text { San Pablo Bay between \#13 \& } \\
\# 14,1.21->\# 5,1.00->\# 4,1.00- \\
>\# E\end{array}$ & $\begin{array}{r}37^{\circ} 59.72^{\prime} \\
-122^{\circ} 23.90^{\prime}\end{array}$ & 3.6 & $2.5 \mathrm{~m}$ barrel $(\mathrm{G}) 1.36$ & $1.25 \mathrm{~m}$ barrel $(\mathrm{G})$ \\
\hline 52 & $020791-2$ & $\begin{array}{l}\text { San Pablo Bay 1.67->\#8, 2.00- } \\
>\# 4,1.35->\# 5\end{array}$ & $\begin{array}{r}38^{\circ} 0.26^{\prime} \\
-122^{\circ} 22.92^{\prime}\end{array}$ & 2.3 & $2.5 \mathrm{~m}$ barrel $(\mathrm{G}) \quad 1.35$ & $\begin{array}{l}1.25 \mathrm{~m} \text { barrel }(\mathrm{G}) \text { eased in- full } \\
\text { organics in catcher }\end{array}$ \\
\hline
\end{tabular}




\begin{tabular}{|c|c|c|c|c|c|c|c|}
\hline $\begin{array}{l}\text { Map } \\
\#\end{array}$ & $\begin{array}{l}\text { Core } \\
\text { Number }\end{array}$ & Setting & $\begin{array}{l}\text { Lat. } \\
\text { Long. }\end{array}$ & $\begin{array}{l}\text { Water } \\
\text { Depth (m) }\end{array}$ & $\begin{array}{l}\text { Type } \\
\text { Core (G) } \\
\text { (B) } \\
\end{array}$ & $\begin{array}{l}\text { Core } \\
\text { length } \\
(\mathrm{m}) \\
\end{array}$ & General Description \\
\hline 53 & $020791-3$ & $\begin{array}{l}\text { San Pablo Bay on grooved } \\
\text { bottom main channel N.E., of } \\
\text { "E", .6-> "E", 1.50->"P", .91- } \\
>\# 5,1.39->\# 7\end{array}$ & $\begin{array}{r}38^{\circ} 1.85^{\prime} \\
-122^{\circ} 23.14^{\prime}\end{array}$ & 11.3 & $3.0 \mathrm{~m}$ barrel $(\mathrm{G})$ & 2.79 & $\begin{array}{l}3.0 \mathrm{~m} \text { barrel }(\mathrm{G}) \text { dropped long } \\
\text { coarse sand layers at top. }\end{array}$ \\
\hline 54 & $020791-4$ & $\begin{array}{l}\text { San Pablo Bay attempt to core } \\
\text { crest of ridge between dune } \\
\text { filled furrows .71->\#9, .52- } \\
>\# 7,1.82->" \text { p" }\end{array}$ & $\begin{array}{r}38^{\circ} 2.15^{\prime} \\
-122^{\circ} 21.50^{\prime}\end{array}$ & 8.2 & $2.5 \mathrm{~m}$ barrel $(\mathrm{G})$ & .94 & sand/mud \\
\hline 55 & $020791-5$ & $\begin{array}{l}\text { San Pablo Bay on bank E. of } \\
\text { Pinole Pt., 1.39->\#9, 2.00- } \\
\text { >\#11 1.70->\#7, 1.30->\#6 }\end{array}$ & $\begin{array}{r}38^{\circ} 1.42^{\prime} \\
-122^{\circ} 19.97^{\prime}\end{array}$ & 2.2 & $2.5 \mathrm{~m}$ barrel $(\mathrm{G})$ & 1.35 & full barrel mud \\
\hline 56 & 020791-6 & $\begin{array}{l}\text { San Pablo Bay bank off } \\
\text { Hercules, 1.52->\#13 3.13->\#7, } \\
1.90->\# 152.21->\# 9,1.70- \\
>\# 11\end{array}$ & $\begin{array}{r}38^{\circ} 1.99^{\prime} \\
-122^{\circ} 18.10^{\prime}\end{array}$ & 2.1 & $2.5 \mathrm{~m}$ barrel $(\mathrm{G})$ & 1.36 & $\begin{array}{l}1.25 \mathrm{~m} \text { barrel }(\mathrm{G}) \text { eased in mud } \\
\text { Ostrea lurida layer at top }\end{array}$ \\
\hline 57 & $020791-7$ & $\begin{array}{l}\text { San Pablo Bay inside end of } \\
\text { Dike \#12, .24-> west end dike, } \\
.59->\# 15, .71->\# 13,1.64->\# 9\end{array}$ & $\begin{array}{r}38^{\circ} 4.06^{\prime} \\
-122^{\circ} 16.81^{\prime}\end{array}$ & 1.7 & $3.0 \mathrm{~m}$ barrel $(\mathrm{G})$ & 2.97 & $\begin{array}{l}\text { full } 3.0 \mathrm{~m} \text { barrel }(\mathrm{G}) \text {, tilted so } \\
\text { top is possibly mixed. }\end{array}$ \\
\hline 58 & $020791-7 a$ & $\begin{array}{l}\text { San Pablo Bay same location } \\
\text { as \#57 }\end{array}$ & $\begin{array}{r}38^{\circ} 4.16^{\prime} \\
-122^{\circ} 16.79^{\prime}\end{array}$ & 1.7 & $1.25 \mathrm{~m}$ barrel $(\mathrm{G}$ & & $\begin{array}{l}1.25 \mathrm{~m} \text { barrel }(\mathrm{G}) \text { eased in to } \\
\text { preserve top full } 4^{\prime}, \text { can see } \\
\text { where mud fell in to top } \sim 7 \mathrm{~cm}\end{array}$ \\
\hline
\end{tabular}

\begin{tabular}{|c|c|c|c|c|c|c|}
\hline \multicolumn{7}{|c|}{$\begin{array}{l}\text { J-2-91-SF } \\
\text { Suisun Bay } \\
\text { Map M }\end{array}$} \\
\hline 59 & 020891-1 & $\begin{array}{l}\text { Suisun Bay coring furrow field } \\
\text { S.W. of Suisun slough } \sim \text { end } \\
\text { of furrow field, } .92->\# 9, .41- \\
>N \text {. bank }\end{array}$ & $\begin{array}{r}38^{\circ} 5.82^{\prime} \\
-122^{\circ} 3.79^{\prime}\end{array}$ & 8.3 & $1.25 \mathrm{~m}$ barrel $(\mathrm{G} .88$ & $\begin{array}{l}1.25 \mathrm{~m} \text { barrel }(\mathrm{G}) \text { dropped } \\
\text { reasonable penetration shells at } \\
\text { top sand layers }\end{array}$ \\
\hline 60 & $020891-2$ & $\begin{array}{l}\text { Suisun Bay field where } \\
\text { furrows are smaller, .74->\#9, } \\
.32->\text { N.bank }\end{array}$ & $\begin{array}{r}38^{\circ} 5.94^{\prime} \\
-122^{\circ} 3.57^{\prime}\end{array}$ & 5 & $3.0 \mathrm{~m}$ barrel $(\mathrm{G}) \quad 1.81$ & $\begin{array}{l}10 \text { ' core dropped pushed the } \\
\text { inner barrel in too far may } \\
\text { have disturbed top a bit. }\end{array}$ \\
\hline
\end{tabular}




\begin{tabular}{|c|c|c|c|c|c|c|c|}
\hline $\begin{array}{c}\text { Map } \\
\#\end{array}$ & $\begin{array}{l}\text { Core } \\
\text { Number }\end{array}$ & Setting & $\begin{array}{c}\text { Lat. } \\
\text { Long. }\end{array}$ & \begin{tabular}{|l|} 
Water \\
Depth (m)
\end{tabular} & \begin{tabular}{|l} 
Type \\
Core (G) \\
(B)
\end{tabular} & $\begin{array}{l}\text { Core } \\
\text { length } \\
\text { (m) }\end{array}$ & General Description \\
\hline
\end{tabular}

\begin{tabular}{|c|c|c|c|c|c|}
\hline $\begin{array}{ll}61 & 020891-3\end{array}$ & $\begin{array}{l}\text { Suisun Bay area of good } \\
\text { furrows seen } 1 / 31 / 91-1141 \text {, } \\
.51->\# 9, .23->\text { N.bank }\end{array}$ & $\begin{array}{r}38^{\circ} 6.11^{\prime} \\
-122^{\circ} 3.81^{\prime}\end{array}$ & $\sim .5$ & $2.5 \mathrm{~m}$ barrel $(\mathrm{G}) 2.08$ & $\begin{array}{l}4 \mathrm{~m} \mathrm{H}_{2} \mathrm{O} \text { seems to have } \\
\text { dropped core on crest } 10^{\prime} \\
\text { dropped reasonable } \\
\text { penetration partial core (but } \\
\text { buried head) }\end{array}$ \\
\hline
\end{tabular}

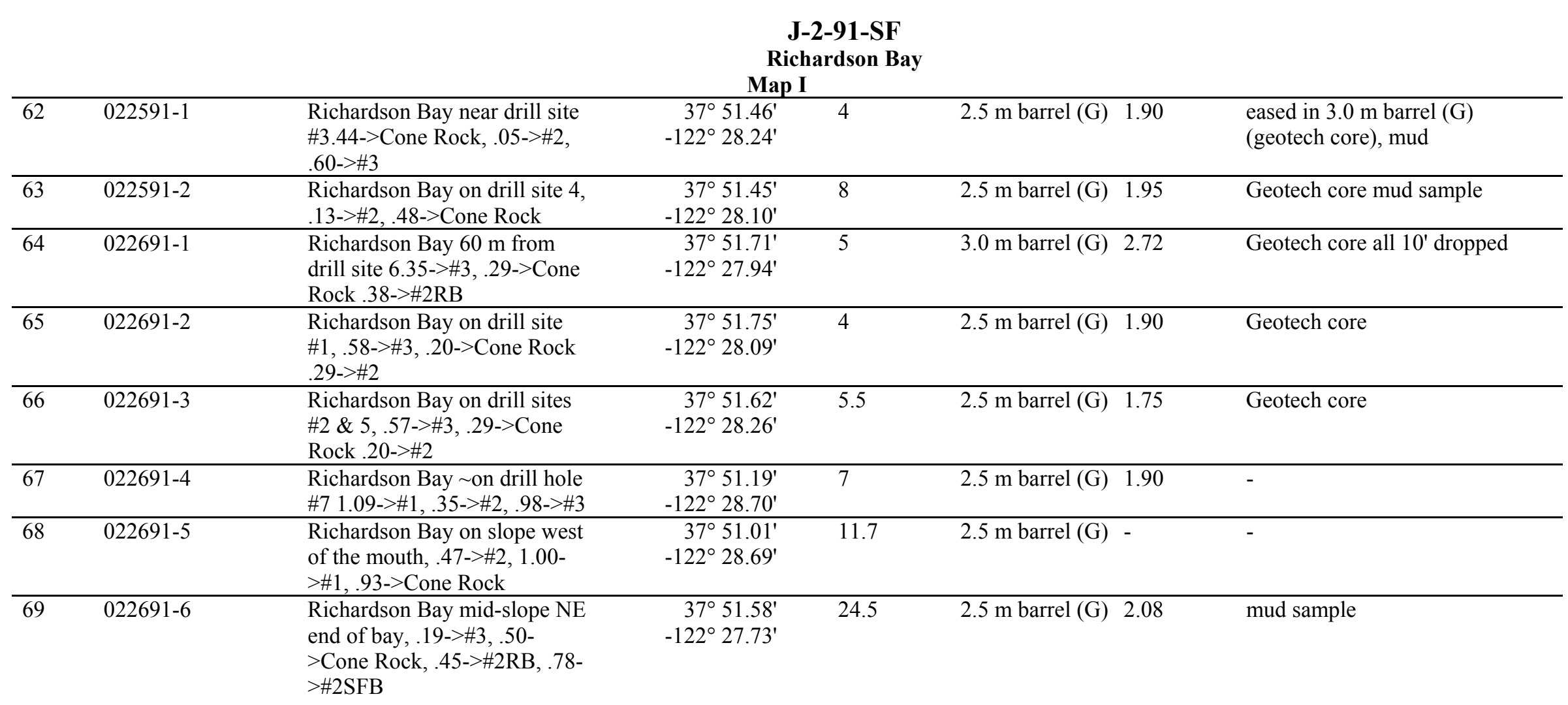




\begin{tabular}{|l|l|l|l|l|l|l|l|}
\hline $\begin{array}{l}\text { Map } \\
\#\end{array}$ & $\begin{array}{l}\text { Core } \\
\text { Number }\end{array}$ & Setting & $\begin{array}{l}\text { Lat. } \\
\text { Long. }\end{array}$ & $\begin{array}{l}\text { Water } \\
\text { Depth (m) }\end{array}$ & $\begin{array}{l}\text { Type } \\
\text { Core (G) } \\
(\mathbf{B})\end{array}$ & $\begin{array}{l}\text { Core } \\
\text { length } \\
(\mathbf{m})\end{array}$ & \begin{tabular}{l} 
General Description \\
\hline
\end{tabular} \\
\hline
\end{tabular}

\begin{tabular}{|c|c|c|c|c|c|c|c|}
\hline 70 & $022691-7$ & $\begin{array}{l}\text { Richardson Bay } 6.5 \mathrm{~m} \text { back end } \\
\text { of D.J. just above platform, } \\
.25->\# 3, .41->\text { Cone Rock .42- } \\
\text { >\#2RB1 .10->\#2SFB }\end{array}$ & $\begin{array}{r}37^{\circ} 51.66^{\prime} \\
-122^{\circ} 27.80^{\prime}\end{array}$ & 7 & $2.5 \mathrm{~m}$ barrel $(\mathrm{G})$ & 2.02 & - \\
\hline 71 & $022691-8$ & $\begin{array}{l}\text { Richardson Bay just down } \\
\text { slope from \# 7, 1.23->\#3, .44- } \\
\text { >\#2RB, .43->Cone Rock .99- } \\
\text { >\#2SFB }\end{array}$ & $\begin{array}{r}37^{\circ} 51.56^{\prime} \\
-122^{\circ} 27.84^{\prime}\end{array}$ & 8.5 & $2.5 \mathrm{~m}$ barrel $(\mathrm{G})$ & 1.83 & - \\
\hline
\end{tabular}

\section{Oakland Area}

\begin{tabular}{|c|c|c|c|c|c|c|}
\hline 72 & $022691-9$ & $\begin{array}{l}\text { Yerba Buena Island East side } \\
\text { of Island on top of platform, } \\
.45->\text { G" } 3 \text { " S. of, bridge, .20- } \\
>\# 4, .29->\text { end of Davy, dock }\end{array}$ & $\begin{array}{r}37^{\circ} 49.35^{\prime} \\
-122^{\circ} 21.27^{\prime}\end{array}$ & 11.5 & $2.5 \mathrm{~m}$ barrel $(\mathrm{G}) \quad 1.48$ & $\begin{array}{l}3.0 \mathrm{~m} \text { barrel }(\mathrm{G}) \text { worms at top } \\
\text { mud }\end{array}$ \\
\hline 73 & $022691-10$ & $\begin{array}{l}\text { Yerba Buena Island on bank, } \\
.45->G^{\prime \prime} 3 ", .34->\# 4, .17- \\
>\text { W.end of Navy, dock }\end{array}$ & $\begin{array}{r}37^{\circ} 49.09^{\prime} \\
-122^{\circ} 21.58^{\prime}\end{array}$ & 14.5 & $2.5 \mathrm{~m}$ barrel $(\mathrm{G}) \sim$ & $\begin{array}{l}\text { Stiff green mud stuck to base } \\
\text { of barrel sandy, limited } \\
\text { penetration }\end{array}$ \\
\hline
\end{tabular}




\section{J-2-91-SF}

\section{Push Cores}

\section{Collected at the Mouth of Richardson Bay}

Map Number: 74

Date 2/19/91

Beginning Depth: $35^{\prime}$ Ending Depth: 40'

$\mathrm{H}_{2} \mathrm{O}$ Depth: 9'

Hole \#: 1

Lat:: $37051^{\prime} 40^{\prime \prime} \mathrm{N}$ Long.: 122028'10" W

Shelby tube \#

1

2

3

4

5

6

7

8

\section{Depth interval}

$15^{\prime} 9^{\prime \prime}$

$20^{\prime} 9^{\prime \prime}$

20'9"-23'3"

23'3"-25'9"

$25^{\prime} 9^{\prime \prime}-28^{\prime} 3^{\prime \prime}$

28'3"-30'9"

36'9"-39'3"

42'2" 
Map Number: 75

Date 2/20/91

Beginning Depth: 13'2" Ending Depth: 38'2"

$\mathrm{H}_{2} \mathrm{O}$ Depth: 14'

Hole \#: 2

Site: 2

Lat.: 37051'32"N Long.: 122028'09"W

\section{Shelby tube}

1

2"-15'8"2

3

4

5

6

7

8

9

10

\section{Depth interval}

$13^{\prime}$

$15^{\prime} 11^{\prime \prime}-18^{\prime} 0^{\prime \prime}$

$18^{\prime} 7 "-20 ' 8 "$

21'6"-23'2"

$23^{\prime} 10^{\prime \prime}-25^{\prime} 8^{\prime \prime}$

24'0"-28'2"

28'4"-30'8"

30'10"-33'2"

33'4"-35'8"

$35^{\prime} 10^{\prime \prime}-38^{\prime} 2^{\prime \prime}$ 
Map Number: 76

Date 2/21/91

Beginning Depth: $14^{\prime} 9^{\prime \prime}$

Ending Depth: $36^{\prime} 10^{\prime \prime}$

$\mathbf{H}_{2} \mathrm{O}$ Depth: 16'10"

Hole \#: 3

Lat.: $37^{\circ} 51^{\prime} 24^{\prime \prime} \mathrm{N} \quad$ Long.: $122^{\circ} 28^{\prime} 07^{\prime \prime} \mathrm{W}$

Shelby tube \#

1

2

3

4

5

6

7

8

9
Depth interval

$$
\begin{aligned}
& \text { 14'9"-16'10" } \\
& \text { 17'4"-19'4" } \\
& 19^{\prime} 6.5 "-21 \text { '10" } \\
& \text { 21'10"-24'4" } \\
& \text { 24'6"-26'10" } \\
& \text { 26'10"-29'4" } \\
& \text { 29'6.5"-31'10" } \\
& \text { 32'2"-34'4" } \\
& \text { 34'8"36'10" }
\end{aligned}
$$


Map Number: 77

Date $2 / 22 / 91$

Beginning Depth: 19'4" $\quad$ Ending Depth: 54'2"

H2O Depth: 19'6"

Hole \#: 4

Lat.: 37051'24" Long.: 122027'58"

Shelby tube \#

1

2

3

4

5

6

7

8

\section{Depth interval}

$$
\begin{aligned}
& 16 ' 10 "-19 ' 4 " \\
& 21 ' 10 "-24 ' 2 " \\
& 26 ' 8 "-29 ' 2 " \\
& 31 ' 8 "-34 ' 2 " \\
& 36 ' 8 "-39 ' 2 " \\
& 41^{\prime} 8 "-44 ' 2 " \\
& 46 ' 8 "-49 ' 2 " \\
& 51 ' 8 "-54 ' 2 "
\end{aligned}
$$


Map Number: 78

Date $2 / 25 / 91$

Beginning Depth: $35^{\prime} 0^{\prime \prime} \quad$ Ending Depth: 52'6"

H2O Depth: 19'4"

Hole \#: 5

Lat.: 37051'32"N Long.: 122027'50"W

Shelby tube \#

1

2

3
Depth interval

35'0"-37'6"

47'6"-50'0"

$50^{\prime} 0 "-52^{\prime} 6 "$ 


\section{Map Number: 79}

Date $2 / 26 / 91$

Beginning Depth: 17'5" Ending Depth: 64'11"

$\mathrm{H}_{2}$ O Depth: $18^{\prime}$

Hole \#: 6

Lat.: $37^{\circ} 51^{\prime} 40^{\prime \prime} \quad$ Long.: 122027'42"

\section{Shelby tube \#}

1

2

3

4

5

6

7

8

9

10

$$
\begin{aligned}
& \text { Depth interval } \\
& \text { 17'5"-19'11" } \\
& 22^{\prime} 5 "-24 ' 11 " \\
& 275^{\prime \prime}-29 ' 11 " \\
& 32 ' 5 "-34 ' 11 " \\
& 37 ' 5 "-39 ' 11 " \\
& 42 ' 5 "-44 ' 11 " \\
& 47 ' 5 "-49 ' 11 " \\
& 52 ' 5 "-54 ' 11 " \\
& 57 ' 5 "-59 ' 11 " \\
& 62 ' 5 "-64 ' 11 "
\end{aligned}
$$


Map Number: 80

Date $2 / 27 / 91$

Beginning Depth: $16^{\prime} 6^{\prime \prime}\left(+5^{\prime}\right)$

Ending Depth: 47'6" $\left(+5^{\prime}\right)$

$\mathbf{H}_{2} \mathrm{O}$ Depth: 18'5"

Hole \#: 7

Lat.: 37051'05" Long.: 122028'21"

\section{Shelby tube \#}

1

2

3

4

5

6

7
Depth interval
$16^{\prime} 6^{\prime \prime}-19^{\prime} 0 "\left(+5^{\prime}\right)$
$21^{\prime} 6 "-24^{\prime} 0 "\left(+5^{\prime}\right)$
$26^{\prime} 6^{\prime \prime}-29^{\prime} 0 "\left(+5^{\prime}\right)$
$31^{\prime} 6 "-34^{\prime} 0 "\left(+5^{\prime}\right)$
$36^{\prime} 6^{\prime \prime}-39^{\prime} 0 "\left(+5^{\prime}\right)$
$41^{\prime} 6 "-44^{\prime} 0 "\left(+5^{\prime}\right)$
$46^{\prime} 6 "-47^{\prime} 6 "\left(+5^{\prime}\right)$ 